\title{
DISCLAMMER
}

This repont was prepared as an sceosst of work sponsored by an neency of the United State Government. Neither the United States Government nor any atency thereof, nor any of their

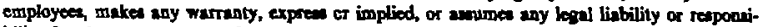
bility for the sceturacy. conpletenese, of wervineas of any information, apparatus, product, of procese discloed, or represents that ite use wodd not infrines privalefy ow oed riphts, Refereace berein to any speciric commetvill product, proceas, or service by trade mame trademurt. manufacturer, or orberwise does not necesarily conatitute or imply its endornement, recommendation, or feyorisg by the United States Gowemment of any afeacy thersol. The views and opinicas of avthon expreased berein do not necesarily alate or reflect those of the Uniled States Goverament or any ugency thereof.

\section{Alpha Particle Density and Energy Distributions in Tandem Mirrors Using Monte-Carlo Techniques}

\author{
John A. Kerns \\ (PH.D. Thesis)
}

Manuscript date: May 1986

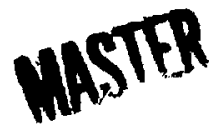

\section{LAWRENCE LIVERMORE NATIONAL LABORATORY University of California - Livermore, California - 94550}

Available from: National Technical Information Service • U.S. Department of Commerce

5285 Port Royal Road - Springfield, VA 22161 • A05 - (Microfiche A01)

4LIATHBUTTEN OF THIS DOCUMENT IS UHUT \& 
ALPHA DARTICLE DENSITY AND ENERGY DISTRIBUTIONS IN TANDEM MIRRORS USING MONTE-CARLO TECHNIQUES

\author{
by \\ John A. Kerns \\ A Thesis Submitted to the Graduate \\ Faculty of Reasselaer Polytechnic Institute \\ in Partial Fulfillment of the \\ Requirements for the Degree of \\ DOCTOR OF PHILOSOPHY \\ Major Subject: Mechantcal Englneering
}

Approved by the

Examining Committee:

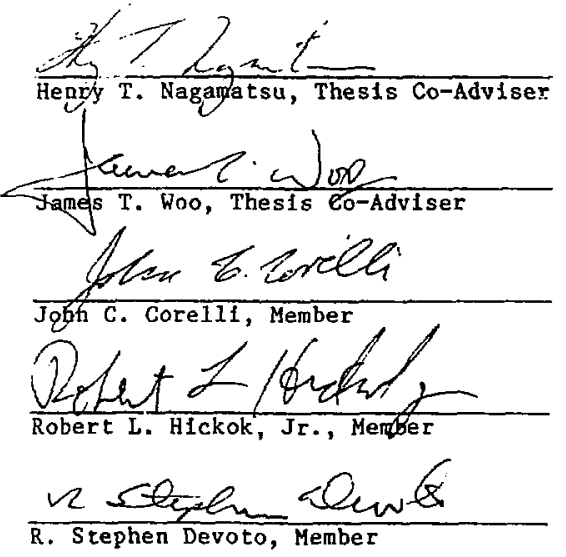

Rensselaer Polytechnic Institute

Troy, New York 


\section{TABLE OF CONTEMTS}

Page

LIST OF TABLES $\ldots \ldots \ldots \ldots \ldots \ldots \ldots \ldots \ldots \ldots \ldots \ldots \ldots \ldots \ldots \ldots$ vi

LIST OF FIGURES $\ldots \ldots \ldots \ldots \ldots \ldots \ldots \ldots \ldots \ldots \ldots \ldots \ldots$ vi

SYMBOL TABLE,$\ldots \ldots \ldots \ldots \ldots \ldots \ldots \ldots \ldots \ldots \ldots \ldots \ldots \ldots \ldots \ldots \ldots$

ACKNOWLEDGMENT $\ldots \ldots \ldots \ldots \ldots \ldots \ldots \ldots \ldots \ldots \ldots \ldots \ldots \ldots \ldots \ldots \ldots$

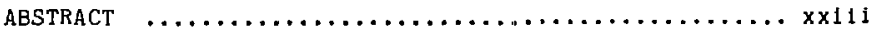

PART I. INTRODUCTION $\ldots \ldots \ldots \ldots \ldots \ldots \ldots \ldots \ldots \ldots \ldots \ldots \ldots \ldots \ldots$ 1

1.1 Background $\ldots \ldots \ldots \ldots \ldots \ldots \ldots \ldots \ldots \ldots \ldots \ldots \ldots \ldots$

1.2 Basic Principles of Tandem Mirror Confinement .... 11

1.3 Physics Issues of Alpha Confinement in

Tandem Mirror Heactors ................. 23

1.4 Methods of Analysis $\ldots \ldots \ldots \ldots \ldots \ldots \ldots \ldots \ldots \ldots$

PART 2. MAGNETIC AND ELECTRIC FIELDS $\ldots \ldots \ldots \ldots \ldots \ldots \ldots \ldots \ldots$

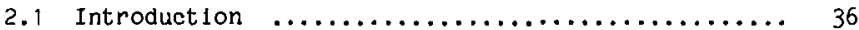

2.2 Tandem Mirror Vacuum Magnetio Fields ......... 37

2.3 Diamagnetic Effects on Magnetic Fields ........ 43

2.4 Axial and Radial Potential Profiles .......... 44

PART 3. GUIDING CENTER THEORY $\ldots \ldots \ldots \ldots \ldots \ldots \ldots \ldots \ldots \ldots \ldots \ldots .47$

3.1 Introduction $\ldots \ldots \ldots \ldots \ldots \ldots \ldots \ldots \ldots \ldots \ldots \ldots \ldots \ldots$

3.2 Classlcai Gulding Center Theory ............ 48

3.3 Classical Drift Simulation $\ldots \ldots \ldots \ldots \ldots \ldots \ldots \ldots$

3.4 Radial Guiding Center Drift $\ldots \ldots \ldots \ldots \ldots \ldots \ldots \ldots$

3.5 Radial Drift Simulation $\ldots \ldots \ldots \ldots \ldots \ldots \ldots \ldots \ldots$ 
PART 4. MONTE CARLO TECHNIQUE

4.1 Introduction $\ldots \ldots \ldots \ldots \ldots \ldots \ldots \ldots \ldots \ldots \ldots \ldots \ldots$

4.2 Alpha Initialization $\ldots \ldots \ldots \ldots \ldots \ldots \ldots \ldots \ldots$

4.3 Alpha Particle Guiding Center Drift .......... 73

4.4 Particle Collisions $\ldots \ldots \ldots \ldots \ldots \ldots \ldots \ldots \ldots \ldots$

4.4.1 Veloc1ty Transformation .............. 76

4.4 .2 Collision Sampling Time ............. 79

4.4 .3 Mass Considerations ................. 80

4.4.4 Drag Collision and simulation .......... 82

4.4.5 Angle Scatter and Dispersion Simulation ... 86

4.4 .6 Collision Model .................... 89

PART 5. PLASMA DENSITY AND TEMPERATURE PROFILES AND

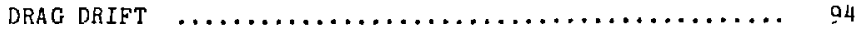

5.1 Introduction $\ldots \ldots \ldots \ldots \ldots \ldots \ldots \ldots \ldots \ldots \ldots \ldots \ldots$

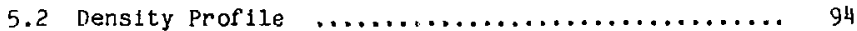

5.3 Temperature Profiles $\ldots \ldots \ldots \ldots \ldots \ldots \ldots \ldots \ldots \ldots$

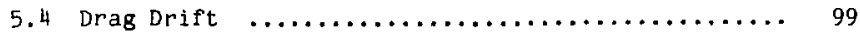

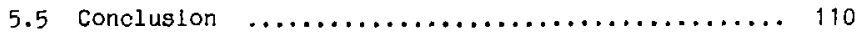

PART 6 . ALPHA PARTICLE THERMALIZATION STUDIES $\ldots \ldots \ldots \ldots \ldots \ldots \ldots$

6.1 Introduction $\ldots \ldots \ldots \ldots \ldots \ldots \ldots \ldots \ldots \ldots \ldots \ldots \ldots \ldots$

6.2 Calculation Verification $\ldots \ldots \ldots \ldots \ldots \ldots \ldots \ldots \ldots$

6.2.1 Alpha Energy Decay ............... 117

6.2 .2 Veloclty Distribution Function $\ldots \ldots \ldots \ldots . \ldots 122$

6.2.3 Energy Distribution Function .......... 133

6.2.4 Gulding Center Drif c Plots ........... 142

6.3 Equal Density and Temperature Scale

Length Results ........................ 142

6.3.1 Generic Reactor Design $\ldots \ldots \ldots \ldots \ldots \ldots \ldots 146$

6.3.1.1 Alpha Particle Thermal1zation $\ldots .146$

6.3.1.2 Alpha Particle Gulding Center

Thermalization .............. 151 
6.3.1.3 Alpha Particle Energy

Distribution ............... 156

6.3 .1 .4 Statistical Analys $1 . . \ldots \ldots \ldots \ldots$ 15y

6.3.2 Variations From the Generlc Reactor

Design $\ldots \ldots \ldots \ldots \ldots \ldots \ldots \ldots \ldots \ldots \ldots$

6.3.2.1 Soale Length Relation for

Alpha Energy Confinement ....... 165

6.3 .2 .2 Varlation of $B, \hat{B}$, and $r_{p}$ for the Single-Scale-Length Analysis .... 167

6.4 Two-Scale-Length Calculation $\ldots \ldots \ldots \ldots \ldots \ldots \ldots 174$

6.4 .1 Varlation of $B, \hat{B}$, and $r_{p}$ for the

Two-Scale-Length Problem .......... 176

PART 7 . DISCUSSION AND CONCLUSION $\ldots \ldots \ldots \ldots \ldots \ldots \ldots \ldots \ldots \ldots \ldots$

PART 8. LiteratuRE CITEd $\ldots \ldots \ldots \ldots \ldots \ldots \ldots \ldots \ldots \ldots \ldots \ldots \ldots \ldots \ldots . \ldots 2$ 
Table 1.1 Comparison of the generic tandem mirror reactor parameters with the Super-Tara and Octopole End Cell designs................................ $3^{4}$

Table 4.1 Velocity changes due to drag for a $3.52 \mathrm{MeV}$ and $60 \mathrm{keV}$ alpha in the reactor core and halo plasmas... 84

Table 6.1 Statistical analys1s of the results for the generic reactor design.............................. 140

Table 6.2 Comparison of Monte-Carlo simulation results varying the density and temperature profiles............. 173

Table 6.3 Comparison of the results for the two-scale-length problem for several values of $:{ }_{\mathrm{pT}} / \mathrm{r}_{\mathrm{pD}} \ldots \ldots \ldots \ldots \ldots, 175$

Table 6.4 Comparison of the results for the two-scale-length problem using sixth- and tenth-power density and temperature profiles with $r_{p T} / r_{p D}=1.1 \ldots \ldots \ldots \ldots .184$ 


\section{LIST OF FIGURES}

Page:

Figure 1.1 Charged particle helical orbit along a magnetio

field line..............................

Figure 1.2 Alpha energy fractions to plasma ions and electrons as a function of plasma electron temperature as

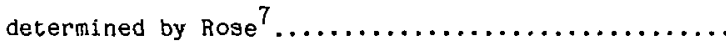

Figure 1.3 Evolution of mirror confinement devices, (A) simple mirror, ( $T$ ' quadrupole fleld generated by Iof fe Bars,

(C) quadr ipole fleld generated by a baseball coil,

(D) quadrupole fleld generated by Yin-Yang coils,

(E) tandem mirror with a simple center cell

connected to quadrupole end cells generated by baseball colls, (F) tandem mirror with thermal barriers, the center cell is a simple mirror, the end cells are made of Yin-Yang colls, and the thermal barrier is generated by baseball colls......

Eigure 1.4 Velocity space losses for, (A) loss cone when the plasma potential is zero, (B) Ion loss region, and (C) electron loss region when there is a plasma potential.............................. 14

Figure 1.5 Vacuum maznetic field axial plasma potentlal and plasma density for a tandem mirror reactor.........

Figure 1.6 Vacuum magnetic field (A), axial plasma potential (B), and plasma density (C) for a tandem mirror with thermal barriers. . . . . . . .

Figure 2.1 Vacuum magnetic field (A), and axial plasma potential (B) for the generic reactor design...... 46

Figure 3.1 Assumed density and temperature profiles for a tandem mirror reactor with a $r_{p}=0.46 \mathrm{~m}$, $n(0)=1.6 \times 10^{14} \mathrm{~cm}^{-3}, T_{i}(0)=40 \mathrm{keV}$, and

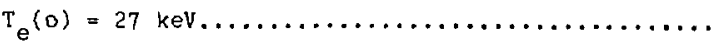

Figure 3.2 Drag profile for the density and temperature profile

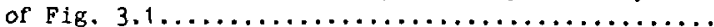

Figure 3.3 Alpha particle orblts in the uniform core plasma and in the drag gradient region between the core and halo. 
Figure 3.4 Drag gradient profile for the density and

temperature profile of Fig. $3.1 \ldots \ldots \ldots \ldots \ldots \ldots \ldots$

Figure 3.5 Relation between the particle position vector $(\bar{r})$ and the guiding center $(\bar{R})$ and Larmor radius $(\bar{\rho})$

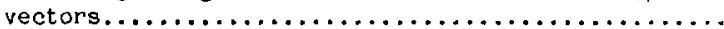

Figure 4.1 Alpha particle radial birth profile determined by Monte-Carlo techniques for 7000 alpha particles $(x)$ and compared with a theoretical

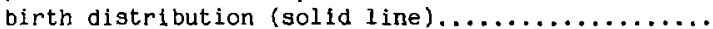

Figure 4.2 Alpha particle radial birth profile determined by Monte-Carlo techniques for 1000 alpha particles $(x)$ and compared with a theoretical birth distribution

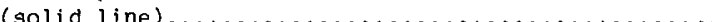

Figure 4.3 Coordinate systems in the center cell of a tandem mirror reactor, Laboratory system $(x, y, z)$, Gulding Center system $\left(\hat{e}_{1}, \hat{e}_{2}, \hat{e}_{3}\right)$, and Particle

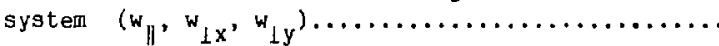

Figure 4.4 Relation between the Laboratory and Particle Coordinate system before and after (primed) a collision.

Figure 4.5 Velocity changes at a collision sampling include magnitude and direction changes $(A)$, which are determined by dispersion (B), and angle scatter, (C) about the mean velocity which is determined by

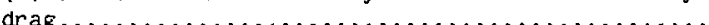

Figure 4.6 The two step collision process first reduces the Larmor radius because of $\mathrm{drag}$ (A) and then incorporates angle scatter and dispersion (B)......

E1gure 4.7 Block diagram of the collision subroutine used in

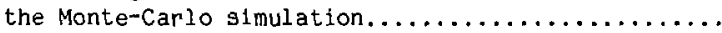

Figure 5.1 Quartic density profile and the modeled quartic profile used in the Monte-Carlo calculation for

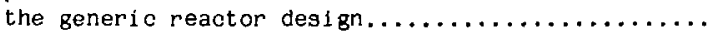

Figure 5.2 Modeled density and temperature profiles for the single-scale-length generic reactor deslgn used ror the Monte-Carlo simulation................. 100 
Figure 5.3 Drag profile for the density and temperature profiles of Fig.5.2...................... 103

Figure 5.4 Drag gradient for the drag profile of Fig. 5.3.... 104

Figure 5.5 Quartic density and step temperature profice used for some reactor designs................. 106

Figure 5.6 Modeled quartic density and step-temperature profile using Eq. 5.2 .4 and 5.3 .1 , respectively..... 107

Figure 5.7 Drag profile for the density and temperature profiles of Fig. $5.6 \ldots \ldots \ldots \ldots \ldots \ldots \ldots \ldots \ldots \ldots \ldots \ldots$

Figure 5.8 Drag gradient for the drag profile of Fig. 5.7.... 109

Figure 5.9 Density and temperature proflle for the two-scale-

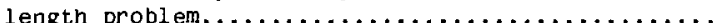

Figure 5.10 Drag profile for the density and temperature

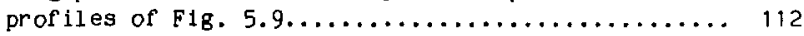

Figure 5.11 Drag gradient for the drag profile of Fig. 5.10... 113

Figure 6.1 Alpha particle energy decay in the hot reacting core plasma $(a-c)$ and in the halo (d). Solid curve is a theoretical prediction given by sivishin 43

Figure 6.2 Fraction of alpha energy given to ions (o) and electrons ( $\square$ ) as a function of plasma electron temperature and compared with Sivukhin ${ }^{43}(-) \ldots \ldots 121$

Figure 6.3 Model of alpha velocity space trajectory through one collision sampling..................... 124

Figure 6.4 Velocity space trajectory from birth to the malization for an alpha with an initlal pitch angle of $90^{\circ}$. Loss cone angle is set to $0^{\circ} \ldots \ldots \ldots \ldots \ldots 126$

Figure 6.5 Velocity space trajectory from birth to thermalization for an alpha with an initial pitch angle of $13^{\circ}$. Loss cone angle is set to $0^{\circ} \ldots \ldots \ldots \ldots \ldots 127$ 
Figure 6.6 Alpha particle normalized distribution function for the generic reactor design. The distribution was produced by 1000 alphas followed frcm $3.52 \mathrm{MeV}$ to $60 \mathrm{keV}$ for $0 \leq r / r_{p}<1 / 3 \ldots \ldots \ldots \ldots \ldots \ldots \ldots \ldots$

Figure 6.7 Alpha particle normalized distribution for the generic reactor design. The distribution was produced by 1000 alphas followed from $3.52 \mathrm{MeV}$ to $60 \mathrm{keV}$ for $1 / 3 \leq \mathrm{r} / \mathrm{r}_{\mathrm{p}}<2 / 3 \ldots \ldots \ldots \ldots \ldots \ldots \ldots \ldots \ldots \ldots 130$

Figure 6.8 Alpha particle normalized distribution for the generic reactor design. The distribution was produced by 1000 alphas followed from $3.52 \mathrm{MeV}$ to $60 \mathrm{keV}$ for $2 / 3 \leq r / r_{p}<r_{w} / r_{p} \ldots \ldots \ldots \ldots \ldots \ldots \ldots$

Figure 6.9 Energy distribution function for the generic reaction design for 1000 alphas followed from $3.52 \mathrm{MeV}$ to $60 \mathrm{keV}$ for $0 \mathrm{~s} \mathrm{r} / \mathrm{r}_{\mathrm{p}}<1 / 3(\mathrm{x})$.

Scl1d line is a theoretical approximation ${ }^{16}$.
Energy distribution function for the generic
reactor design for 1000 alphas followed from
$3.52 \mathrm{MeV}$ to $60 \mathrm{keV}$ for $1 / 3 \leq \mathrm{r} / \mathrm{r}<2 / 3(\mathrm{x})$. Soldd line is a theoretical approximation ${ }^{16} \ldots \ldots \ldots 136$

Figure 6.11 Energy distribution function for the generic reactor design for 1000 alphas followed from $3.52 \mathrm{MeV}$ to $60 \mathrm{keV}$ for $2 / 3 \leq \mathrm{r} / \mathrm{r}_{\mathrm{p}} \leq \mathrm{r}_{\mathrm{w}} / \mathrm{r}_{\mathrm{p}}(\mathrm{x})$. Solid line is a theoretical approximation ${ }^{16} \ldots \ldots \ldots 137$

Figure 6.12 Energy distribution function predicted by MonteCarlo techniques $(x)$ and by Driemeyer ${ }^{16}(-\ldots)$ for an alpha in a uriform plasma at $10 \mathrm{keV} . . . \ldots .141$

Figure 6.13 Alpha guiding center position after one collision sampling time for the generic reactor design...... 143

Figure 6.14 Alpha gulding center position after 25 collisions sampiing cimes for the generic reactor design...... 144

Figure 6.15 Aipha gulding center position after 50 collislons siruplang times for the generic reactor design...... $14 \mathrm{~s}$

Figure 6. . Thermal alpha particle radial profile normalized to the total number of alphas followed, for the generic reactor design................... $1:$; 
Figure 6.17 Thermal alpha radial density profile normalized to the total number of alphas followed divided by the plasma yolume, for the generic reactor design............................... 149

Figure 6.18 Alpha particle thermalization rate for the generic reactor design..................... 152

Figure 6.19 Number of alpha particles born minus the number of alpha particles thermalizing in $r_{j} \ldots \ldots \ldots \ldots 153$

Flgure 6.20 Alpha partlc]e(x) and gulding center(o) normalized thermal densities as a function of radius......... i54

Figure 6.21 Alpha partisle( $x$ ) and gulding center(o) normalized blrth densities as a function $*$ radius.......... 157

Figure 6.22 Normalized alpha energy deposition profile for the generic reactor design................. 158

Figure $6.2 \%$ Alpha energy deposition profile per unit volume coralized to the total energy deposited in the total plasma volume......................... 160

Figure 6.24 Alpha power given to the plasma in the generic

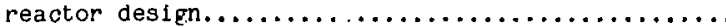

Figure 6.25 Alpha power given $\mathrm{k}$ : plasma ions and electrons In the seneric reaction deslgr................ 162

Figure 6.26 Alpha particle dist.-1button to the not reacting core, halo, and velocity space loases as a function of $\rho / r_{c}$ for the singie-scale-length problem. The subserlpt $\mathrm{j}$ refers to the core,

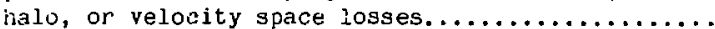

Figure 6.27 Alpha energy distribution to the hot reacting core, halo, and relocity space losses as a function of $\rho / 1$ for the single-scale-length problem. The subscrigt $j$ refers to the core, halo, or velocity space losses.............. 170

Figure 6.28 Modified density proflle for the single-scalelength problen......................... 172

Figure 6.29 Alpha power to the plasma for the two-scalelength problem when $r_{p T} / r_{p D}=1.1 \ldots \ldots \ldots \ldots \ldots \ldots \ldots 177$ 
Figure 6.30 Alpha power to the plasma for the two-scalelength problem when $\mathrm{r}_{\mathrm{pT}} / \mathrm{r}_{\mathrm{pD}}=1.2 \ldots \ldots \ldots \ldots \ldots \ldots, 178$

Figure 6.31 Alpha energy losses out 0 . the loss cone for the two-scale-length problem for $\mathrm{r}_{\mathrm{pT}}{ }^{\prime \mathrm{r}}{ }_{\mathrm{pD}}=1.1 \ldots \ldots \ldots .181$

Figure 6.32 Alpha energy losses to the halo for the two-scalelength problem for $r_{p T} / r_{p D}=1.1 \ldots \ldots \ldots \ldots \ldots \ldots, 183$ 


\section{LIST OF SYABOLS}

\begin{tabular}{|c|c|}
\hline$A_{D}$ & Constant in Spitzer's collision model \\
\hline$v$ & Constant in the radial drag gradient \\
\hline $\overrightarrow{\mathrm{B}}$ & Magnetic field vector \\
\hline$v$ & vacuum magnetic field vector \\
\hline B & Magnitude of the magnetic field \\
\hline a & $\begin{array}{l}\text { Magnitude of the magnetio field including } \\
\text { diamagnetic effects }\end{array}$ \\
\hline $\mathrm{c}$ & $\begin{array}{l}\text { Magnitude of the magnetic fleld in the center cell } \\
\text { of a Tandem Mirror }\end{array}$ \\
\hline II & $\begin{array}{l}\text { Magnitude of the magnetic field in the mirror of a } \\
\text { Tandem Mirror }\end{array}$ \\
\hline $\max$ & Maximum magnetlc field in a mirror device \\
\hline min & Minimuin magnetic fleid in a mirror device \\
\hline $\mathrm{v}$ & Magnitude of the vacuum magnetic field \\
\hline$x, B_{y}, B_{z}$ & $\begin{array}{l}\text { Components of the magnetic field in Laboratory } \\
\text { coordinates }\end{array}$ \\
\hline & $\begin{array}{l}\text { Arbitrary constant in the theoretical energy } \\
\text { distribution function }\end{array}$ \\
\hline$-D$ & Deuterium - Deuterium \\
\hline$-\mathrm{T}$ & Deuterium - Tritium \\
\hline$\hat{e}_{1}, \hat{e}_{2}, \hat{e}_{3}$ & $\begin{array}{l}\text { Unit vectors in the Gulding Center coordinate } \\
\text { system }\end{array}$ \\
\hline$e_{j}$ & Energy per unit volume in radial bin $j$ \\
\hline $\bar{E}$ & Electric field vector \\
\hline E & $\begin{array}{l}\text { Electric field parallel to the magnetic field } \\
\text { General force field vector }\end{array}$ \\
\hline & Drab force \\
\hline
\end{tabular}




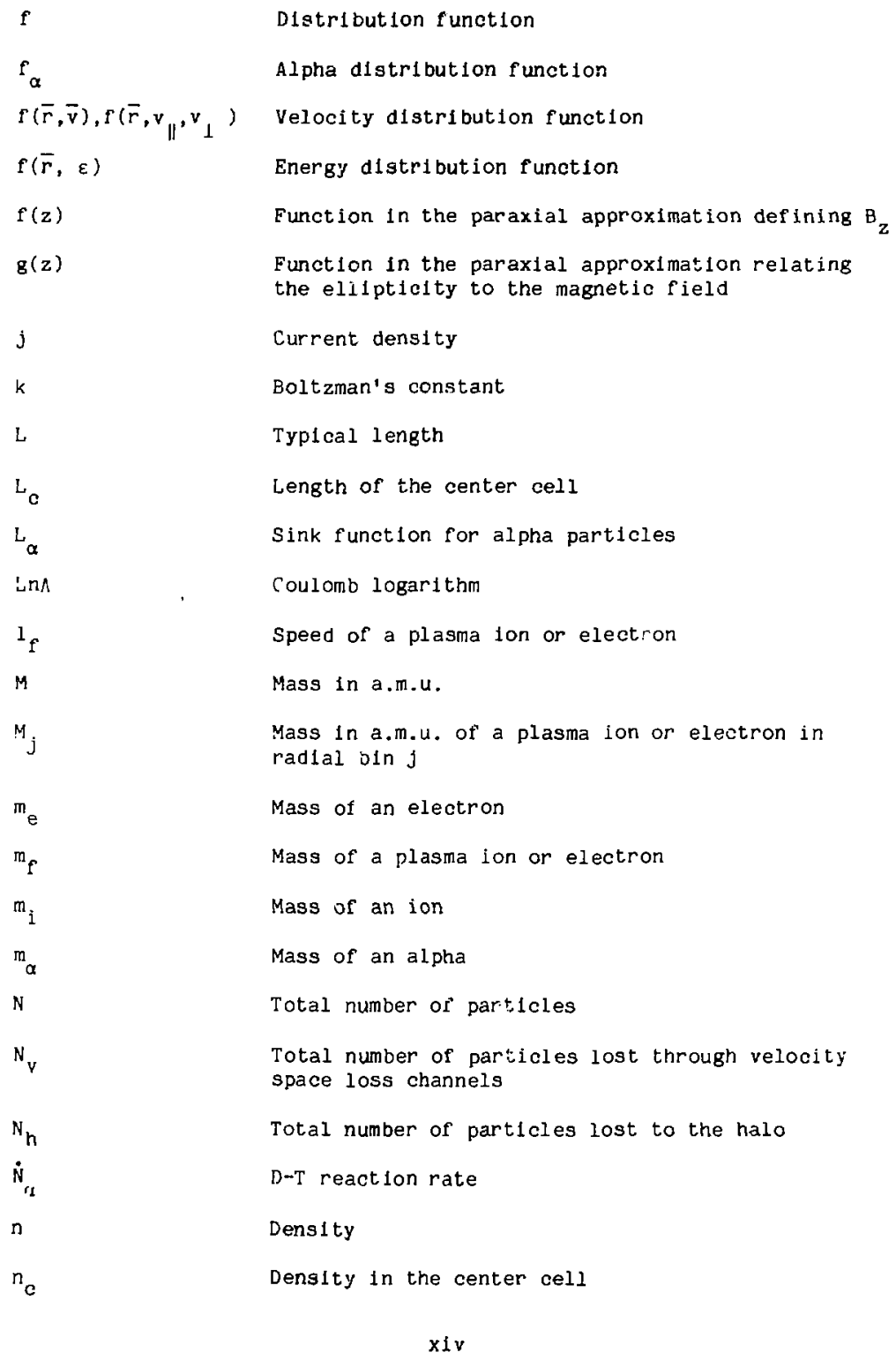




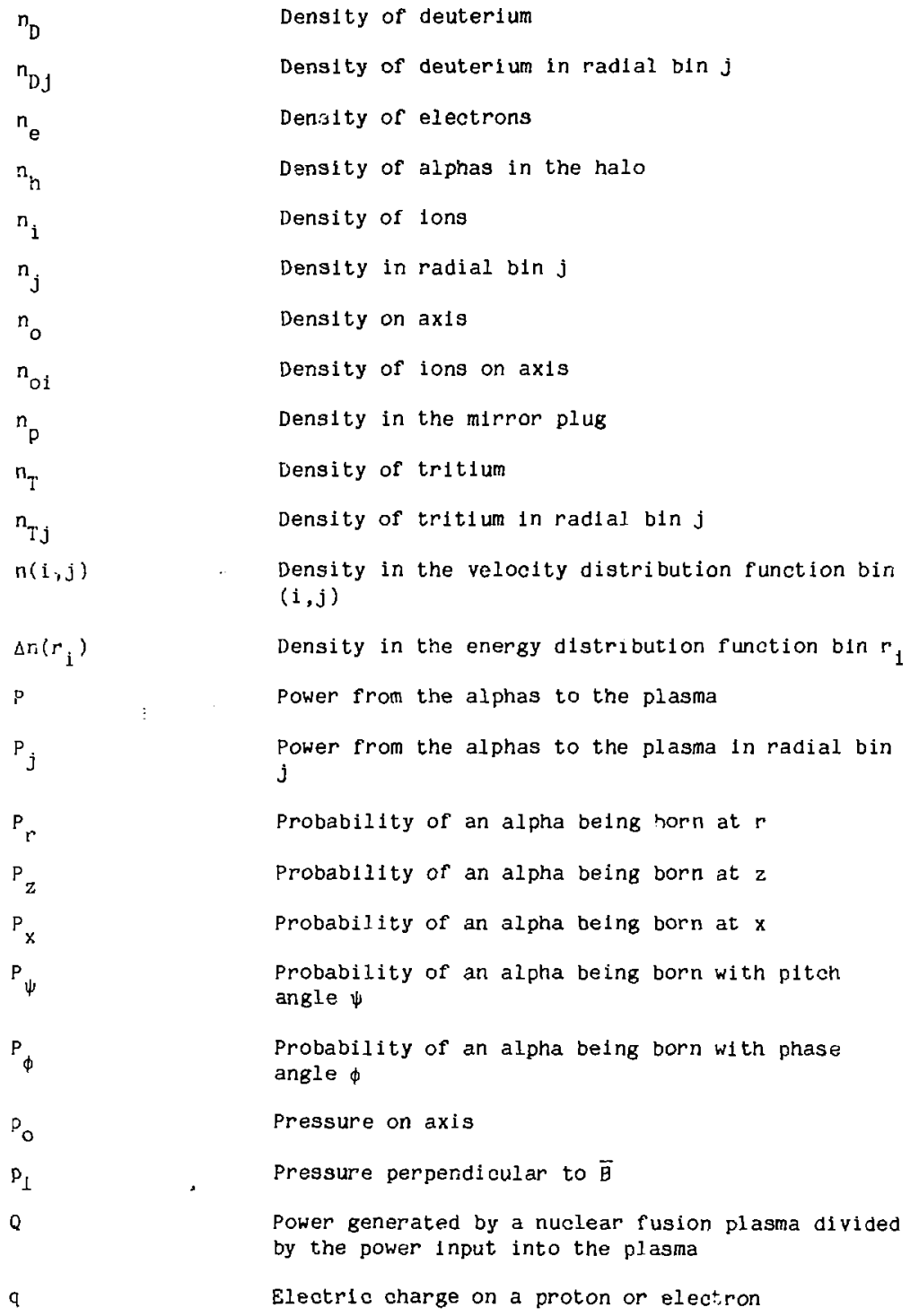




\begin{tabular}{|c|c|}
\hline$q_{\alpha}$ & Electric charge on an alpha \\
\hline$\overline{\mathrm{R}}$ & Guiding center position vector \\
\hline$\dot{\bar{R}}_{D}$ & Guiding center drift velocity \\
\hline R & Magnitude of the guiding center position vector \\
\hline$R_{1}$ & Random number \\
\hline$\overline{\mathrm{r}}$ & Particle position vector \\
\hline$r$ & Magnitude of the particle position vector \\
\hline$r_{c}$ & Hot reacting plasma radius \\
\hline$r_{p}$ & Input plasma radius \\
\hline$r_{\mathrm{pD}}$ & Scale length for density \\
\hline$r_{\mathrm{pT}}$ & Scale length for temperature \\
\hline $\mathrm{S}_{\alpha}$ & Source function for alpha particles \\
\hline $\mathrm{T}$ & Temperature \\
\hline $\mathrm{T}_{\mathrm{e}}$ & Electron temperature \\
\hline${ }^{\mathrm{T}} \mathbf{i}_{\mathrm{i}}$ & Ion temperature \\
\hline$T_{0}$ & Temperature on axis \\
\hline $\mathrm{T}_{\text {oe }}$ & Electron temperature on axis \\
\hline $\mathrm{t}$ & Time \\
\hline$\Delta t$ & Time between collision sampling events \\
\hline$\Delta t(i, j)$ & $\begin{array}{l}\text { Accumulated time of the alphas that passed through } \\
\text { the velocity space bin }(i, j)\end{array}$ \\
\hline$\Delta t\left(r_{1}\right)$ & $\begin{array}{l}\text { Accumulated time of the alphas that passed through } \\
\text { the energy distribution bin }\left\langle\mathrm{r}_{i}\right)\end{array}$ \\
\hline$\Delta \mathrm{T}$ & $\begin{array}{l}\text { Accumulated time of all the alphas that } \\
\text { contributed to the velocity distribution function }\end{array}$ \\
\hline v & Veloolty \\
\hline $\bar{v}_{D}$ & Drift velocity \\
\hline $\bar{v}_{\alpha}$ & Alpha velocity \\
\hline
\end{tabular}




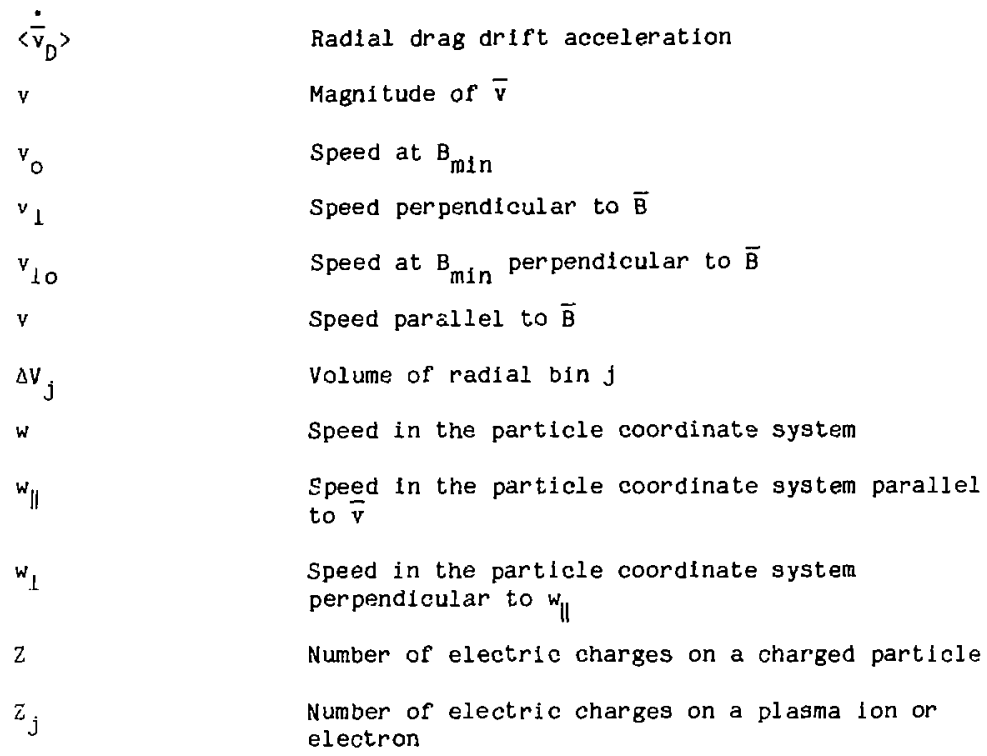

Radial drag drift acceleration

Magnitude of $\bar{v}$

Speed at $B_{m i n}$

Speed perpendicular to $\overrightarrow{\mathrm{B}}$

Speed at $B_{\min }$ perpendicular to $\bar{B}$

Speed parallel to $\overrightarrow{\mathrm{B}}$

Volume of radial bin $\mathrm{j}$

Speed in the particle coordinate system

Speed in the particle coordinate system parallel to $\overline{\mathrm{v}}$

Speed in the particle coordinate system perpendicular to $w_{\|}$

Number of electric charges on a charged particle

Number of electric charges on a plasma ion or electron

\section{GREEK SYMBLS}

Alpha particle

Ratio of plasma pressure to magnetic field pressure

Peak beta

A.rerage beta

Beta as a function of $r$

Variable denoting the power of $r / r_{p}$ in density and temperature profiles

Power of $\left(1-\frac{r}{r_{p D}}\right)$ in the modeled density profile Power of $\left(1-\frac{r}{r_{\mathrm{pT}}}\right)$ in the modeled temperature
profile

Expansion parameter equal to $\mathrm{p} / \mathrm{L}$ 


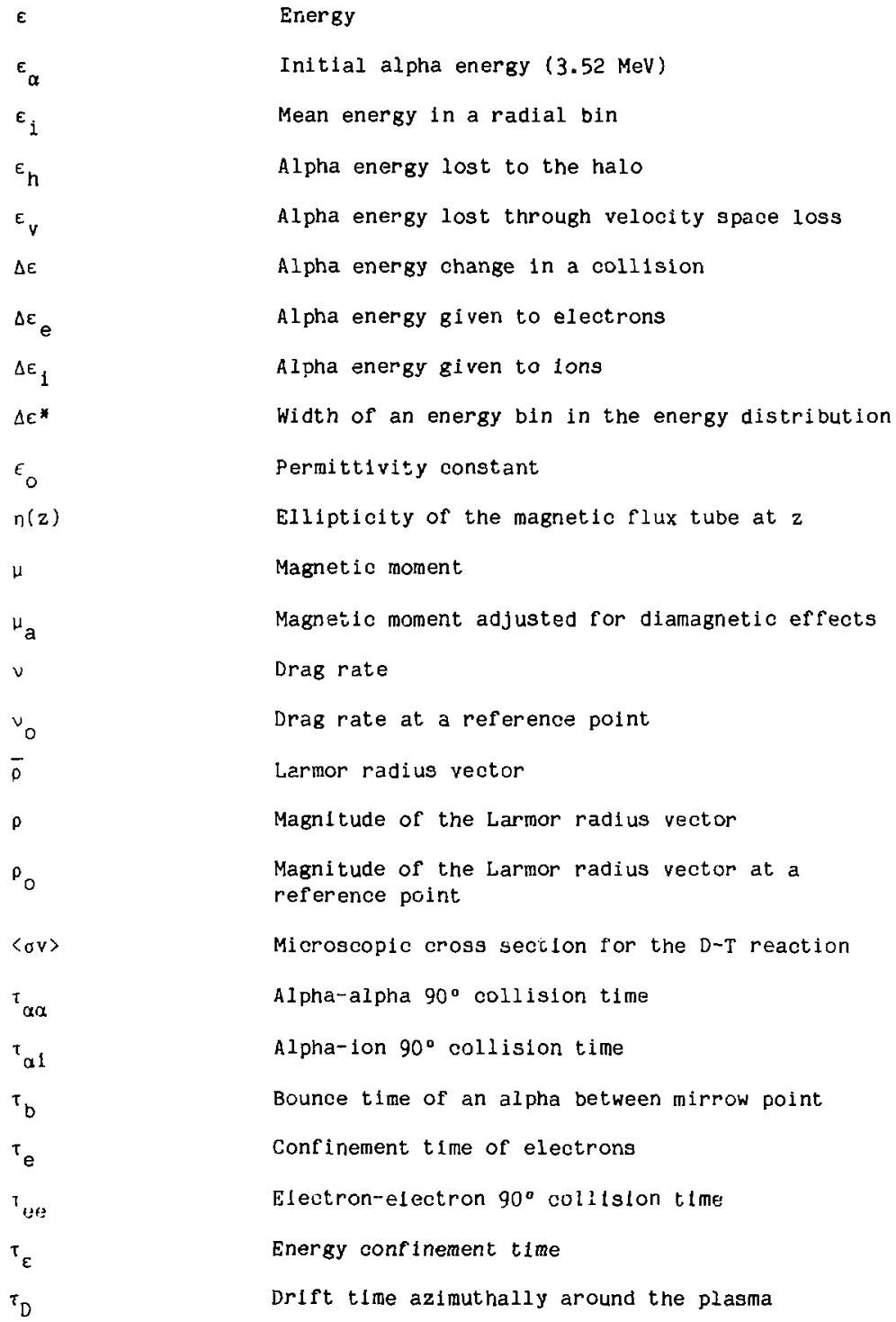

Initial alpha energy (3.52 MeV)

Mean energy in a radial bin

Alpha energy lost to the halo

Alpha energy lost through velocity space loss

Alpha energy change in a collision

Alpha energy given to electrons

Alpha energy given to lons

Width of an energy bin in the energy distribution

Permittivity constant

Ellipticity of the magnetic flux tube at 2

Magnetic moment

Magnetic moment adjusted for diamagnetic effects

Drag rate

Drag rate at a reference point

Larmor radius vector

Magnltude of the Larmor radius vector

Magnitude of the Larmor radius vector at a reference point

Microscoplc cross section for the $D-T$ reaction

Alpha-alpha $90^{\circ}$ collision time

Alpha-ion $90^{\circ}$ collision time

Bounce time of an alpha between mirrow point

Confinement time of electrons

Electron-electron $90^{\circ}$ colilston time

Energy confinement time

Drift time azimuthally around the plasma 


$\begin{array}{ll}{ }_{\perp} & \text { Alpha } 90^{\circ} \text { gcattering time } \\ { }^{\top} \| & \text { Alpha dispersion time } \\ \Phi_{p C} & \text { Electrostatic potential } \\ \Phi(x) & \text { Plug to center cell potential } \\ \phi & \text { Error function } \\ x^{\prime} & \text { Phase angle } \\ \psi & \text { Azimuthal angle } \\ \psi^{\prime} & \text { Pitch angle } \\ \psi_{\phi} & \text { Logs cone pitch angle } \\ \omega_{C} & \text { Magnetic flux } \\ & \text { Cyciotron Irequency }\end{array}$

$\begin{array}{ll}\text { a } & \text { Actual } \\ \text { b } & \text { Bounce } \\ \text { D } & \text { Cyclotron or center cell } \\ \text { D } & \text { Drift or Deuterium } \\ \text { e } & \text { Deuterium in bin } \mathrm{j} \\ \mathrm{ee} & \text { Electron } \\ \mathrm{f} & \text { Electron-electron } \\ \mathrm{h} & \text { Field particle } \\ \mathrm{i} & \text { Halo } \\ \mathrm{j} & \text { Ion } \\ \mathrm{max} & \text { Radial bin } \mathrm{j} \\ \mathrm{min} & \text { Maximum } \\ & \text { Minimum }\end{array}$




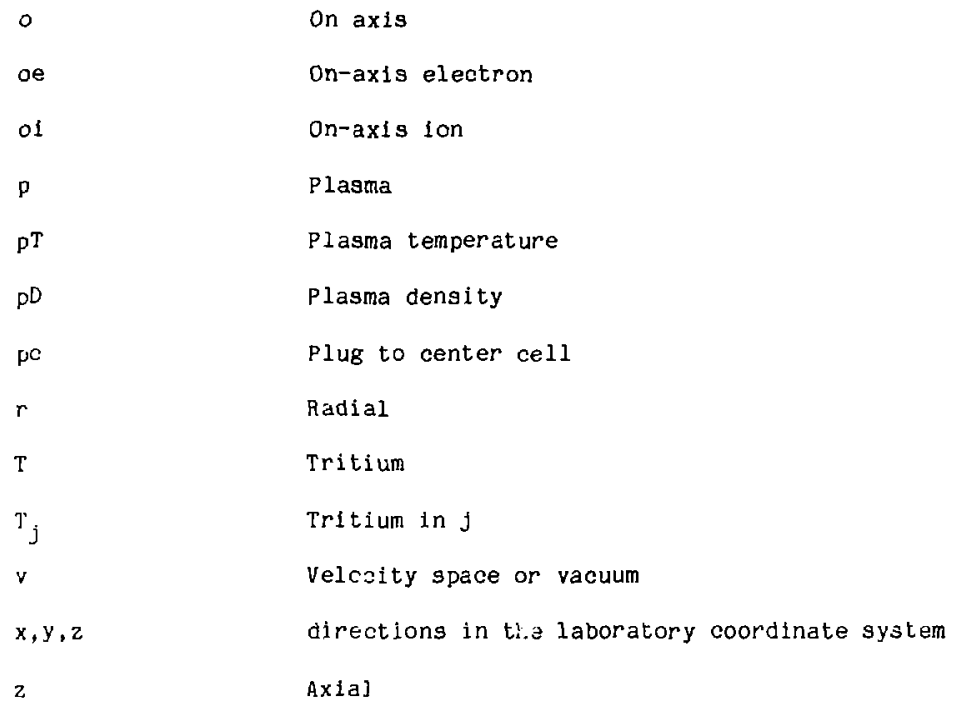

on axis

on-axis electron

On-axis ion

Plasma

Plasma temperature

Plasma density

Plug to center cell

Radial

Tritium

Tritium in $j$

Velcaity space or vacuum

directions in ti.s laboratory coordinate system

Axial

Perpendicular

Parallel

\section{GAEEK SUBSCAIPTS}

Alpha particle

Alpha-alpha

alpha-ion

Energy

Azimuthal angle

Phase angle

P1tch angle

\section{SYMBOLS SUBSCRIPTS}




\section{ACKNOWLEDGMENTS}

This thasis is the cu?mination of a long reseisreh effort in which many people have contributed ideas, suggestions, and moral support; I appreciate all of this help. I speciflcally want to thank Professor Fredrick F. Ling, Chalrman of Mechanical Engineering, Aeronautical Engineering and Mechanics at Rensselaer Polytechnic Institute for giving me the opportunity and encouragement to start and finish this task. I am grateful to Dr. James $T$. Woo who has been a cochairman of this committee since its inception and who has helped guide my efforts to this conclusion. I am also grateful to Dr. R. Stephen Devoto who has taken the time and effort at Lawrence Livermore National Laboratory to answer questions, ask questions and make suggestions for this work. I also thank Dr. Henry T. Nagamats who has assumed the responsibility of being a co-chairman of my thesis committee at essentlally the last minule. I would alscilke to thank the other members of my commIttee Professor Robert L. Hickock and Professor John C. Corelli.

The preparation of this final document has been handled expertly by several people and I thank all of them for their efforts. I wish to acknowledge the efforts of Vergie Zuppan for her contribition, since she has been responsible for completing the final iterations of this document on a challenging computer system.

Finally, I wish to especlally thank my wife, Peg, for her unwaivering support and encouragement throughout this research project. Without Peg's support, my research work would have been imposilble. I 
also thank my daughter, Laura, and son, Mike, for thelr patience in the many hours we have been apart because of this work.

I also acknowledge and appreclate the flnancial support and computer time given to me by Rensselaer Polytechnte Institute, Lawrence Livermore National Laboratory, and the Department of Energy. 


\section{ABSTRACT}

Alpha particles, born at $3.52 \mathrm{MeV}$, are used to maintain the ignition temperatures in a deuterium-tritium (D-T) reacting plasma above $10 \mathrm{keV}$ in tandem mirror reactors. The alpha energy is transferred to the plasma by Coulomb collisions and, after thermalization, the alphas can accumulate in the plasma and quench the reaction. We have simulated the alpha thermalization process using a Monte-Carlo technique, in which the alpha guiding center is followed between simulated collisions and Spitzer's collision model is used for the alpha-plasma interaction. Monte-Carlo techniques are used to determine the alpha radial birth position, the alpha particle position at a collision, and the angle scatter and dispersion at a collision. The plasma is modeled as a hot reacting core, surrounded by a cold halo plasma ( $T-50 \mathrm{eV}$ ). Alpha orbits that intersect the halo loose $90 \%$ of their energy to the halo electrons because of the halo drag, which is ten times greater than the drag ir the core. The uneven drag across the alpha orbit also produces an outward, radial, guiding center drift. This drag drift is dependent on the plasma density and temperature radial profiles. We have modeled these profiles and have specifically studied a single-scale-length model, in which the density scale length $\left(r_{\mathrm{pD}}\right)$ equals the temperature scale length $\left(\mathrm{r}_{\mathrm{pT}}\right)$, and a two-scale-length model, in which $\frac{r_{\mathrm{PD}}}{r_{\mathrm{PT}}}=1.1$. Alpha energy losses to the core and halo were determined by varying the vacuum magnetic field $\left(B_{v}\right)$, peak beta 
$(\vec{B})$, and plasma radius $\left(r_{p}\right)$ for each profile combination. The energy losses to tre halo are a function of $\frac{\rho}{r_{c}}$ where $\rho$ is $\frac{0.27}{B_{v}[1-\langle B(r)\rangle]^{1 / 2}}$, and $r_{c}$ is the reacting core radlus. When $\frac{p}{r_{c}}>0.3$ for the singlescale-length study, the energy losses to the halo are greater than $40 \%$. This occurs for $\frac{\rho}{r_{c}}>0.39$ for the two-scale-length study, but there is a $50 \%$ varlation in the data for $\frac{\rho}{r_{c}}>0.35$ in this case. This data scatter suggests that $\frac{\rho}{r_{c}}$ is not an exact scaling parameter. Until improvements are made to the scaling parameter, the alpha halo losses must be calculated using plasma density and temperature profiles that produce equilibrium alpha distributions. 


\section{PART 1}

\section{INTRODUCTION}

\section{$1.1 \quad$ Background}

The prospect of power generation from themonuclear fusion, which can operate on a virtually unlimited deuterium fuel suppiy has been the subject of extensive research and developmert efforts $f$ or over thirty years. 1,2 In the fusion process, light nucleii are combined in a reaction that releases an amount of energy equal to the mass difference between the reactants and products. The light nucleil are brought together to distances equal to their nuclear radij by overcoming the coulomb repulsive forces between them. Therefore, the reacting particles must be sufficiently energetic for the fusion reactions to occur. In a thermonuclear reactor, these energetic particles are to be contained at sufficiently high density and for times sufficiently long for significant fusion reactions to occur. Because these reacting particles are energetic, they will exist in a fully lonized state as a plasma. The abllity to confine and sustain a plasma of 1 ight ions at thermonuclear conditions has been the subject of much of the efforts in controlled fusion research up to the present time.

Although nuclear fusion reactions involving hydrogen atoms are the souince of energy of our sun and of all the stars in the universe, the fusion reactions involving hydrogen are too slow to duplicate in the laboratory for power generation. ${ }^{3}$ other reactions, such as the deuterium-deuterlum (D-D) reaction which has two reacting branches, 


$$
\begin{aligned}
D+D & +n(2.45 \mathrm{MeV})+3 \mathrm{He}(0.82 \mathrm{MeV}) \\
& +\mathrm{p}(3.03 \mathrm{MeV})+\mathrm{T}(1.01 \mathrm{MeV})
\end{aligned}
$$

and the detuerium-tritium (D-T) reaction,

$$
D+T \rightarrow n(14.06 \mathrm{MeV})+{ }^{4} \mathrm{He}(3.52 \mathrm{MeV}) \text {, }
$$

have much larger reaction cross sections and are therefore easier to produce. Of all the known fusion reactions, the $D-T$ reaction has the largest maximum cross section $\left(5 \times 10^{-24} \mathrm{~cm}^{2}\right)$ which peaks at the lowest relatlve energy (100 kev). Deuterlum is a hyorogen isotope that occurs naturally in seawater in the ratio of one part in 6500 , and therefore there is an ample supply that is readily avallable. Tritium, which is radioactive with a half 1 ife of approximately nine years, does not occur naturally, but can be generated in the exothermic reaction,

$$
\mathrm{n}+\mathrm{Li}+\mathrm{T}(2.7 \mathrm{MeV})+{ }^{4} \mathrm{He}(2.1 \mathrm{MeV}) \text {. }
$$

Since the $D+T$ reaction produces an energetic neutron and $l$ ithium is also an abundant element, it is possible to produce a power-generating fusion reactor plant based on the $D-T$ reaction. Furthermore, the alpha particle ( $\left.{ }^{4} \mathrm{He}\right)$, which is the other reaction product, is born with $3.52 \mathrm{MeV}$ energy and it will thermalize with the D-T plasme by Coulomb interactions. It is therefore possible to sustain a continuous burning D-T plasma at thermonuclear conditions by alpha heating, a condition known as ignition when no additional energy input is required. Because of these possibilities, the emphasis of the international program to develop fusion power is being directed mainly on reactor concepts operating on the D-T fuel cycle. 4 
At present, there are two principal apprcaches to controlled thermonuclear fusion. In one approach, known as inertial onnfinement, a small D-T fuel pellet is heated by either laser or particle beams to achieve a substantlal D-T burn before the reaction is quenched by expansion cooling. The advantage of this approach is its relative simplicity. However, since the reaction time is limited to the disassembly time of the reacting plasma, the complete process, including initial heating, must be accomplished on a nanosecond time scale. The disadvantage of this approach, therefore, is the need to deliver a large amount of energy to the pellet on a very short time scale and at a rate fast enough to produce a significant amount of energy for power generation. Most of the research in inertial confinement fusion is based upon understanding the physics associated with the absorption of high energy pulses into the cuel pellet and in developing the necessary high energy sources for dellvering these pulses.

The other approach, known as magnetic confinement fusion, attempts to confine the hot reacting plasma in vacuun, away from material walls, by using elfetromagnetic fields. Since the plasma particles are charged, their orbits, in terms of their position vector $(\bar{r})$ as a function of time $(t)$, are governed by the equation of motion,

$$
\ddot{r}=\frac{q}{m}(\bar{E}+\bar{v} \times \bar{B}),
$$

where $\bar{E}$ and $\bar{B}$ are the electric and magnetic fields in the plasma due to 
both external (imposed) and internal (self-consistent) sources, $q$ is the charge on the particle, and $m$ is its mass. It is well known that in a slowly varying magnetic field, the magnetic moment,

$$
\mu=\frac{1}{2} \frac{m v_{1}^{2}}{B} \text {, }
$$

where $v_{\downarrow}$ is the velocity perpendicular to $\bar{B}$, is an approximate constant of the motion, $1 . e$, an adiabatic invariant. Therefore, a charged particle can be effectively "tied" to a helical orbit about a field line, as shown in Fig. 1.1 .

This fact suggest two types of magnetic field configurations for the confinement of a thermonuclear plasma. One is to bend magnetic field lines such that they close on themselves, and the system is effectively endless. This lype of configuration is a "closed system", and the principal approach is the tokamak concept in which the magnetic flux bundle is bent into a torus to confine the plasma. The disadvantage of this configuration is that the curvature of the field lines gives rise to a particle drift out of the confinement system. These curvature effects are compensated by techniques which result in limiting the plasma pressure that can be stably confined. The other type of field configuration depends on $\mu$ being an adiabati invariant and on the fact that a steady magnetic field can not impart energy to the particle; therefore, its energy,

$$
\epsilon=\frac{1}{2} m v^{2}
$$




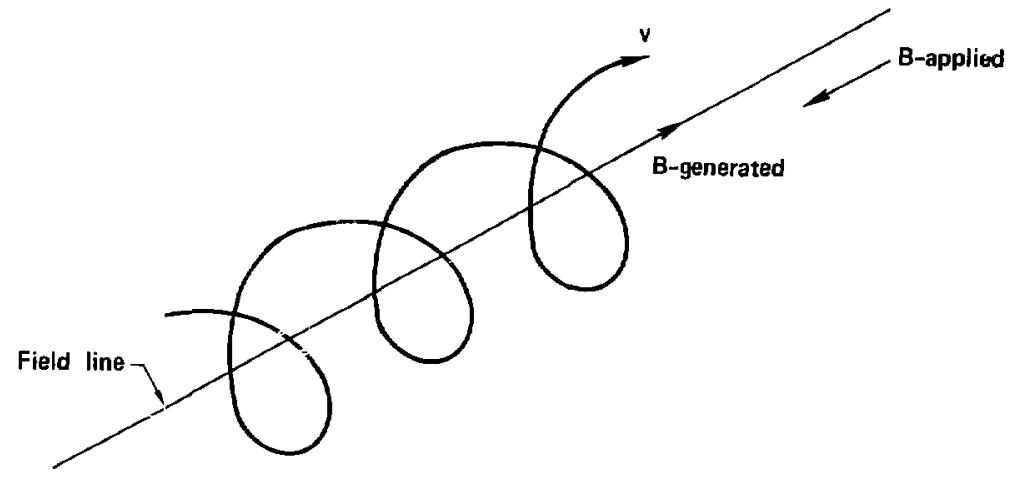

Figure 1.1 Charged particle helical orbit along a magnetic field
line 
is also constant. Particles with sufficiently small parallel motion along the field lines and situated in a region between two field maximums w1l be trapped in such a magnetic "well." This type of confinement system, which is usually linear to avoid the vertical drift, is known as a magnetic mirror, or "open system" because the field lines extend beyond the confinement region. The disadvantage of a magnetic mirror is that for a given mirror ratio,

$$
R=\frac{B_{\text {max }}}{B_{\text {min }}},
$$

where $B_{\max }$ and $B_{\min }$ are the value of the maximum and mintmum magnetic fleld in the system, only those particles with sufficiently large pitch angles at the field minimum are trapped. Particles that are magnetically trapped/confined have pitch angles ( $\psi)$ larger than $\psi^{\prime}$, which is defined by

$$
\sin ^{2} \psi^{\prime}=\frac{v^{2}}{v^{2}}=\frac{1}{R}
$$

where the perpendicular and parallel velocity components are defined with respect to the direction of the magnetic fleld. Therefore, tre mLror system is leaky because particles are continuously scattered through all pitch angles, and they wlll be lost in an average sense in $\Rightarrow$ characteristlo scattering time. For this reason, simple magnetic mirror confinement is not expected to lead to an economically 
attractive fusion reactor. However, interest in magnetic mirror conf inement has been revived with the invention of the taisdom mirror concept. 5.6 In 1 ts most simple form, the tandem mirror consists of three linked magnetlc mirror cells, with the center cell maintained at a Lower potentlal than the two end cells. The two end cells serve as electrostatic end plugs for the plasma confined in the center cell. In principle, the confinement in the center cell can be arbitrarily scaled up by increasing the potential difference, and fusion yield can be also be arbitrarily raised by increasing the length of the center cell. With this enhancement, the tandem mirror is one of the malnilne approaches for magnetically confined fusion.

In both the inertial and magnetic confinement approaches to controlled fusion, the plasma is heated by the $3.52 \mathrm{MeV}$ alpha particles from the D-T reaction. This heating is a critical lssue in determinfing the performance of the system. In the case of inertial confinement, the alpha particle energy deposition determines the fractional burn of the fuel pellet and, therefore, the energy gain that can be realized from each shot. In the case of magnetic confinement, the condition for ignition is determined by the balance of plasma heating by the alpha particles against energy losges through all channels. For this reason, the confinement and slowing down of $3.52 \mathrm{MeV}$ alpha particles in a variety of magnetic confinement systems has been the subject of a number of previous studieg concerned with both basic physics and specific fusion reactor designs. 
The basic physics of energetic alpha particle slowing down in a homogeneous equilitrium plasma can be calculated from consideration of charged particle interaction via Coulomb forces. An approximate calculation for the fraction of energy transferred to the electrons and ions in a thermal hydrogen plasma as a function of the plasma temperature has been given by Rose, ${ }^{7}$ and his result is shown in Eig. 1.2. To sustain the fusion reaction in the plasma, it is necessary to maintain the fons hot. At temperatures in expess of $30 \mathrm{kev}$, a greater portion of the alpha energy is transferred directly to the 1ons. At temperatures below $30 \mathrm{keV}$, however, most of the energy is deposited with the electrons. The ions are then heated indirectly by the electrons. The partition of the alpha energy in a fusion reactor, therefore, depends on the transport and confinement lifetime of the electrons, ions, and alpha particles in the specific system.

There have been many studies of alpha particle conflnement and thermalization in tokamak systems, ${ }^{8-15}$ ranging from determination of the energy distribution functions, ${ }^{12}$ to effect of alpha bombardment on reactor walls 9,15 and proposals for improving alpha energy confinement by changing the reactor designs. ${ }^{15}$ These studies, and many more, form a substantial body of knowledge on plasma heating by alpha particles in tokamaks. There have also been detailed studies of alpha thermalization in alternate fusion reactor concepts. Driemeyer ${ }^{16}$ made a complete study of fusion product effects in field reversed mirrors. Potok, et al. 17 studied alpha particle confinement in torsatron type of reactors. Knowledge on the thermalization of alpha particles in tandem 


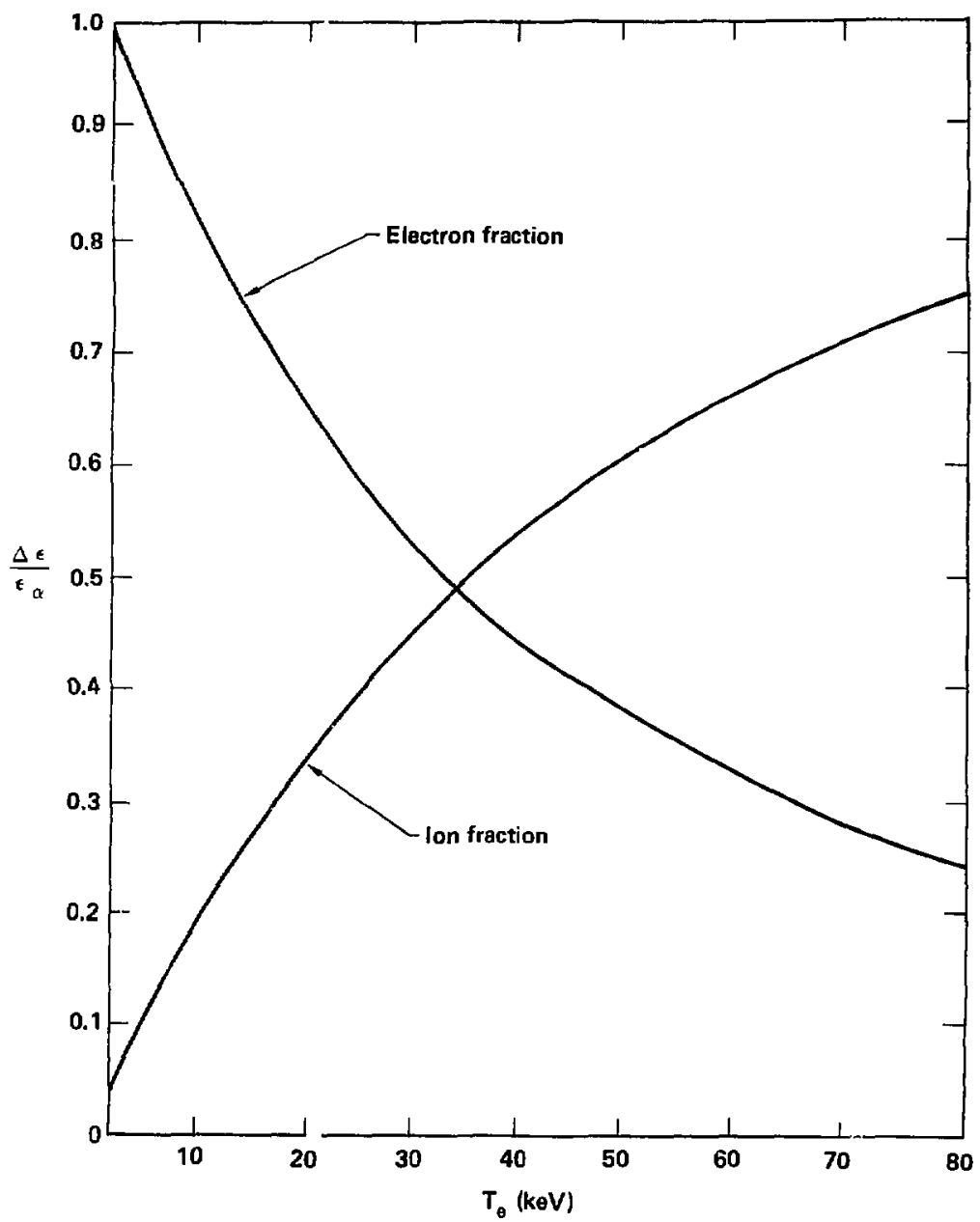

Figure 1.2 Alpha energy fractions to plasma lons and elestrons as

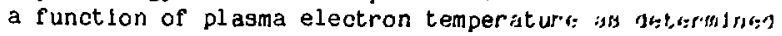
by Rose ${ }^{7}$ 
mirror reactors, however, is much less complete. This is largely due to the fact that alpha particle confinement in tandem mirror reactors is a more complicated subject involving, in the most general case, three-dimensional fields in which many variations are possible. The three-dimensional character of the confinement field can lead to varying degrees of enhanced radial diffusion similar to neoclassical transport assoatated with fteld line curvature in closed confinement systems.

An initial study of alpha particle confinement in tandem mirrors has been performed at Lawrence Livermore National Laboratory (LI,NL). ${ }^{18}$ This study evaluated basic considerations for alpha particle confinement in tandem mirror systems, but it reglected neoclassical diffusion effects. Neoclassical diffusion in tancem mirrors is important when there are significant nonaxisymmetric components of the magnetic field in the center cell. Effects of neoclassical diffusion on alpha confinement was later addressed by Devoto and Ohnishi ${ }^{19}$ and by Devoto, et al. ${ }^{20}$ with the conclusion that that approximately $60-80 \%$ of the alpha energy is available for deposition in the plasma. These results were based on following alpha trajectories in a specific field configuration and determining a radial confinement life time by evaluating the radial displacement of the orbits. An analytical solution of the alpha energy deposition in a square-well magnetic mirror fleld with a uniform plasma background nad been ubtained by Santarius and Callen ${ }^{21}$ with glmilar numerical results. 
In more refined models of tandem mirror reactors, 1 t was reeognized that the hot, reacting plasma core must be surrounded by a Low temperature halo plasma. $22,23,24$ Thls halo plasma can be very effective in shielding the hot core from charge exchange losses with the background neutrals, and therefore has a profound effect on the equilibrium properties of the core. Furthermore, recent advances in the tandem mirror concept have led to designs of more axisymmetric center cells to minimize neoclassical transport losses and the introduction of thermal barriers to isolate the hot electron end plug from the reacting fusion plasma in the center cell. To evaluate the ajpha particle thermalization in such systems, we need a baslc understanding of the key physics processes affecting alpha energy deposition in tandem mirror systems, as well as a general technique for evaluating the effect of these processes in specific tandem mirror configurations. The present thesis is concerned with a study of alpha particle transport and energy deposition in tandem mirror reactors by using the Monte-Carlo technique. The objective $1 \mathrm{~s}$ to 1 dentify and take into account all the important effects in the alpha energy deposition process and develop scaling relations useful for tandem mirror reactor designs. The technique, in the form of a computer code, is also sufficiently general to allow for evaluation of specific cases.

\subsection{Basic Principles of Tanden Mirror Confinement \\ In order to address the issues of alpha particle confinement and energy deposition in tandem mirror reactors, we need to first nave a clear understanding of the principles of tandem mirror confinement.}


The present tandem mirror concept is a sophisticated embodiment of basic magnetic mirror confinement physics. It.s complex design and many possible variations can be best understood by reviewing the evolutionary chain of magnetic mirror confinement devices. The magnetic mirror trap, in its most simple form, consists of two solenoldal coils separated along their common axis, as shown in Fig. 1.3a. This design produces an azimuthally symmetric field with an axial minimum at the midplane between the two colls. As has been noted, particles are trapped between the two mirror coils when their midplane pitch angle is greater than the critical value given by Eq. (1.1.5). The critical angle $\psi^{\prime}$, rotated azimuthally, forms two opposing conical reglons in velocity space as ghown in Fig. 1.4a. These regions are known as the loss cone of a mirror trap within which particles are not confined. Particles with velocities outside the loss cone region will remain confined until their velocity is scattered into the loss cone at random by coulomb collisions with other charged particles.

In addition to these velocity space losses, the simple nirror trap suffers from two serious problems. The first problem occurs because the surface of the plasma near the midplane, where the bulk of the plasma is confined, is concave. In the ideal MHD limit, tho confining fleld lines conform to the plasma surface. Therefore, for concave surfaces, adjacent field lines in the vacuum region outside the plasma surface occupy a volume larger than the volume inside the surface which is occupled by the plasma. It can be shown that because 


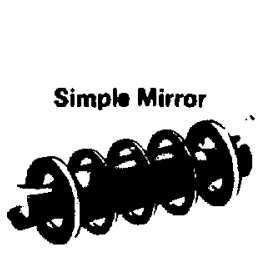

A

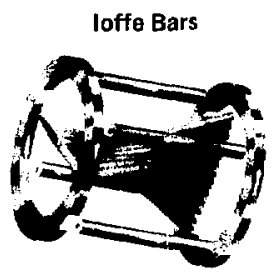

B

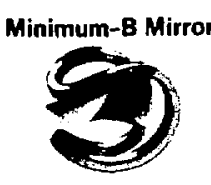

C

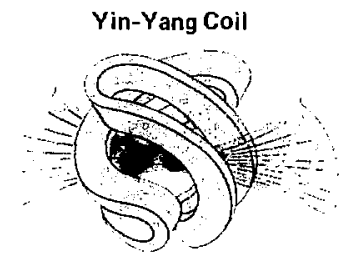

D

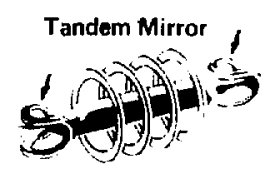

E

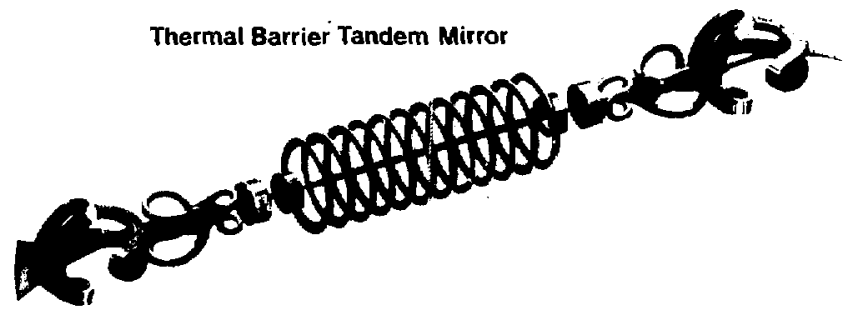

F

Figure 1.3 Evolution of mirror confinement devices, (A) simple mirror, (B) quadrupale field generated by loffe bars $(C)$ quadrupole field generated by a baseball coil, (D) quadrupole field generated by Yin-Yang coils, (E) tandem mirror with a simple center cell connected to quadrupole end cells generated by basebail coils, (F) tandem mirror with thermal barriers, the center cell is a simple mirror, the end cells are made of Yin-Yang coils, and the thermal barrier is generated by baseball coils. 

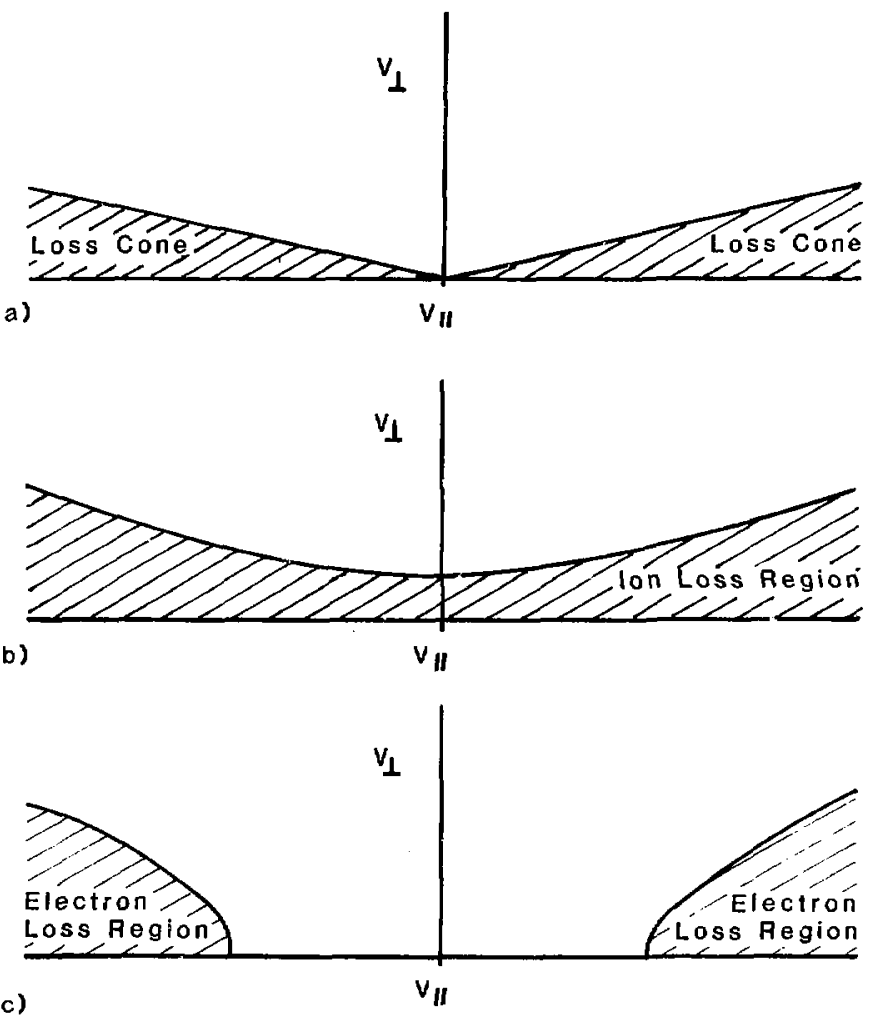

Figure 1.4 Velocity space losses for, (A) loss cone when the plasma potential is zero, (B) iol, loss region, and (C) electron loss region when there is a plasma potantial 
of this difference, it is energetically posstble for the plasma to exchange position with the vacuum reglon. Thls prceess can occur in the form of a nydromagnetic instabllity that leads to rapid loss of the confined ptasma. The instability can be suppressed if the plasma surface is convex such that this interchange is energetically impossible.

Convex field lines imply that the magnitude of the magnetic field must increase away from the surface. This realization leads to the development of the minimum-B trap, the slmplest form of which is obtained by adding a set of quadrupoles to the simple mirror, as shown in $\mathrm{Fig}$. 1.3b. With this arrangement, the absolute magnitude of the confining field rises both axially away from the mid plane, because of the mirror coils, and radially away from the axis, because of the quadrupoles whose contributions are exactly cancelled on axis. Mii stable confinement by such a minimum-B mirror field was first demonstrated by Iof $\mathrm{e}^{25}$ in the U.S.S.R. A number of variations of the quadrupole minimum-B configurations are possible; most nctable are the so-called baseball, shown in Fig. $1.3 \mathrm{c}$, and the yin-yang coil system, shown in Fig. 1.3d. It is also possible to use higher order multipoles, which differ from the quadrupole fields, because the radial fleld cancellation occurs over a radial region around the axis, dur to nigher order cancellations of the field.

Although the minimum-B configuration is able to eliminate the MHD stability problein, there remains a second problem associated with magnetic mirror confinement. Because of the existence of the loss 
cone, particles are confined, on average, in the mirror trap for only one collisional scattering time. The electrons nave higher thermal speed, since their mass is smaller than the ions, and are scattered more frequently than the ions. As a result, the electrons are scattered into the loss cone at a higher rate, leading to the development of a positive space charge due to charge imbalance. In the presence of this space charge, electrons are electrostatically confined, and only the very energetic electrons escape while the ions are accelerated out by the positive potential, such that the low energy ions are not confined at all. By taking the electrostatic potential built up in mirror plasmas by this electron loss into consideration in the energy conservation, the loss boundary in velocity space is given by

$$
\frac{v^{2}}{v_{0}^{2}}=\left(1+\frac{2 q \Phi}{m}\right) / R \text {, }
$$

where $q$ is positive for lons and negatlve for electrons, and $\phi$ is the potential difference. This is the equation of a hyperboloid of a single surface for the lons and of two surfaces for the electrons, as shown in Figs. $1.4 \mathrm{~b}$ and 1.4c, respectively. As a result, the electron loss cone is essentially eliminated, and the electron distribution can relax to closely approximate a Maxwellian distribution, while the ion $10 s 3$ cone is greatly enlarged, and the confined fon distribution is vold of low energy particles. It has been shown by Pastukov ${ }^{26}$ that 
because of the presence of the potential well, electron confinement is enhanced by the exponential power of the ratio of the potential well depth to the temperature of the species; the conflnement time is given by

$$
\tau_{e}=\frac{\sqrt{\pi}}{2} \tau_{e e} \frac{2 R+1}{R} \operatorname{Ln}(4 R+2) \frac{\Phi}{T_{e}} \exp \frac{\phi}{T_{e}},
$$

where $\tau_{e}$ is tre electron-pitch-angle scattering time and $T_{e}$ is the electron temperature. For the ions, however, their distribution possesses an inverted population and is potentially unstable. Instabilities of this type, which are referred to as microinstabilfties because they depend on the distribution for their excitation, have been observed in virtually every relevant, simple, mirror experiment. A result of these instabilities, in which the free energy is released, is that the ions fill in the vold space near the velocity space origln where they are not contained. Therefore, basic mirror confinement suffers from microinstabilities, which further reduces the confinement time that can be attained.

A technique for suppressing the mi crolnstability was demonsirated in the $2 X$-IIB experiment 27 at LLNL by flowing a warm plasma stream into the plasma which fills in the loss cone. Although this technique supresses the microinstabilities, it can be shown that the minimum-B confinement cannot be used by itself for a viable fusion reactor. The limitation for minimum-B confinement is that the energy gain, or $Q$, for this system is less than 2, whereas reactor $Q$ values 
should be greater than 5. Therefore, it was concluded in the mid 1970 's that basic mirror confinement is not scalable to an economically attractive fusion power reactor and some alternative approach was needed.

The tandem mirror confinement concept was a timely invention conceived independently by Fowler and Logan ${ }^{5}$ in the U.S, and Dimov, et. al. ${ }^{6}$ in the U.S.S.R to meet this need. They reasoned that because the electrons are well confined by the potential well, their distribution is essentially in collisional equilibrium. Therefore, if one links three mirror cells together, as shown in Fig. i.3e, the electron distribution will follow the Boltzmann well relation,

$$
\Phi=T_{e} L N \frac{n_{p}}{n_{c}} .
$$

If the center cell is maintained at a lower density than the two end cells, then the potential will have a similar axial profile, as shown in Fig. 1.5. Thus, while the electrons are electrostatically confined by the positive space charge potentlal throughout the system, the ions in the center cell are also electrostatically confined by the hizher potential in the two end celis. According to the Pastukhov result, the Ion confinement time in the center cell will be enhanced by the exponential factor of the ratio of the Ion confinement potential, " $\mathrm{pc}$ ' to the ion temperature, $T_{1}$, which can be arbitrarlly scaled up by increasing the helght of the potential barrier. The two end cells, whlch serve as electrostatic end plugs, still operate much as simple 


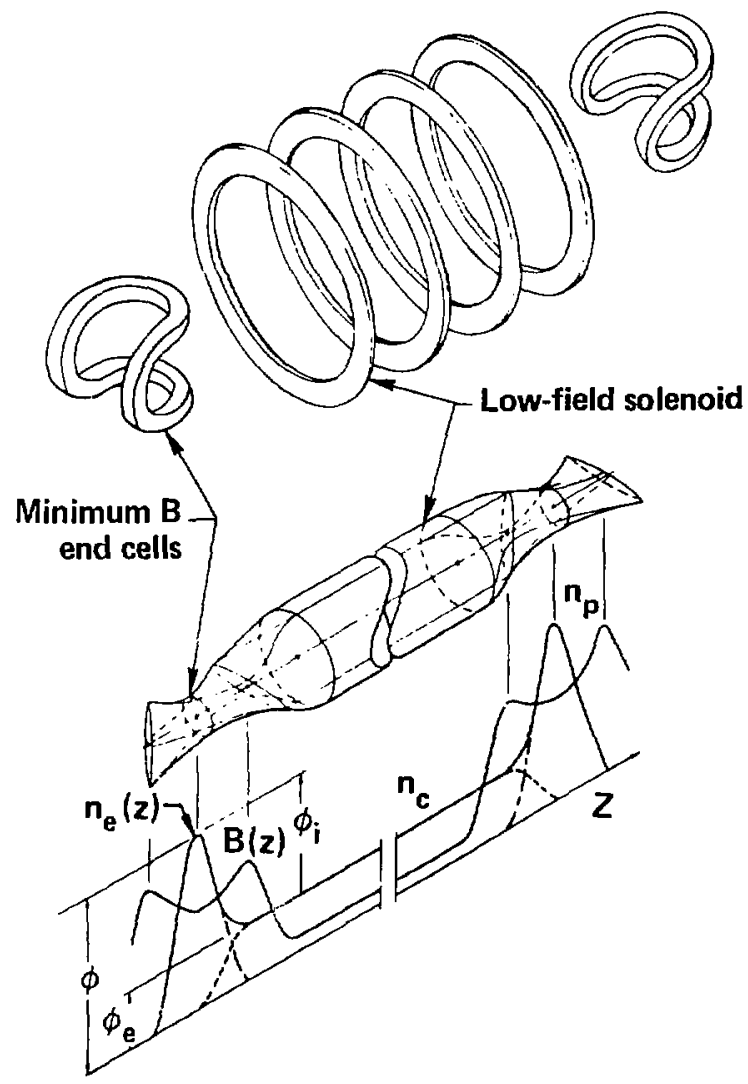

Eigure 1.5 Vacuum magnetic field axial plasma potential and plasma density for a tandem mirror reactor 
mirror cells. By using minimum-B configuration in the end cells, the ent1re system can be made MHD stable. Ions confined in the center cell which are able to overcome the potentlal barrier will be lost by streaming through the end cells. This streaming plasma serves to flil the loss cone region of the plasma confined in the end plugs, which suppresses the excitation of microinstabilities. Therefore, the system can be sustained by neating and fueling the end plugs against coulomb scattering losses. Furthermore, since the center cell has neutral MHD stability, it can be made arbitrarily long to increase the volume of the reacting fusion plasma, whlch increases the fusion power output. This system, which is known as a tandem mirror in the U.S. and as ambipolar trap in the U.S.S.R., is a very attractive basis for a thermonuclear fusion reactor.

Although in principle the tandem mirror concept is attractive for thermonuclear plasma conf inement, there are several practical difficulties. First of all, the nonaxisymmetric quadrupole fields in the anchor, which are required for MHD stability, perturb the perfect axisymmetry of the solenoidal center cell field. The finite azimuthal magnetic fleld component created by the quadrupole fields causes larye radial excursion of the confined particles. Ryutov and Stupekov 28

first pointed out that such exoursions can $g$ lye rise to enhanced radial diffusion, which limits the radial confinement time that can be realized in such tandem mirror conflgurations. In order to eliminate this enhanced diffusion, the magnetlc fleld in the center cell must be made fully axisymmetric. Secondly, in order to develop a sufficiently 
large potential barrfer, the density in the plug must be much greater than in the center cell. Since the power required to sustaln the tandem mirror configuration is proportional to the plug density, very high input power is required to develop the potential necessary for confinement. The magnetic fleld in the plug must also be high to contain the plug density, which complicates the quadrupole magnet design. Because the magnitude of the amblpolar potential in a mirror confined plasma is determined by the electron temperature, the barrier can be enhanced if the electrons in the plug can be heated independent of the center cell. This lead Baldwin and Logan 29 to propose the thermal barrier tandem mirror, In which a potential minimum is introduced between the plug and the center cell, whicn limits the electron conduction between the two regions. A typical coil set for this mirror system is shown in FIg. 1.3f; the axial magnetic and potential profiles for this configuration, which became known as thermal barrier tandem mirrors, are shown in Fig. 1.6. To make the magnetic field in the center cell axisymmetric, a number of proposals have also been advanced. These include the use of choke coils to recircularize the quadrupole flelds, as in most of the new tandem mirror designs, such as Gamma-10 in Japan and TARA and MFTF-B in the U.S. The use of more or fully axisymetrlc anchors generated by using hexipoles, cusps, E-rings and pondermotive forces to provide MHD stabllity to the system have also been proposed. The current trend in tandem mirror reactor design, therefore, generally involves an 

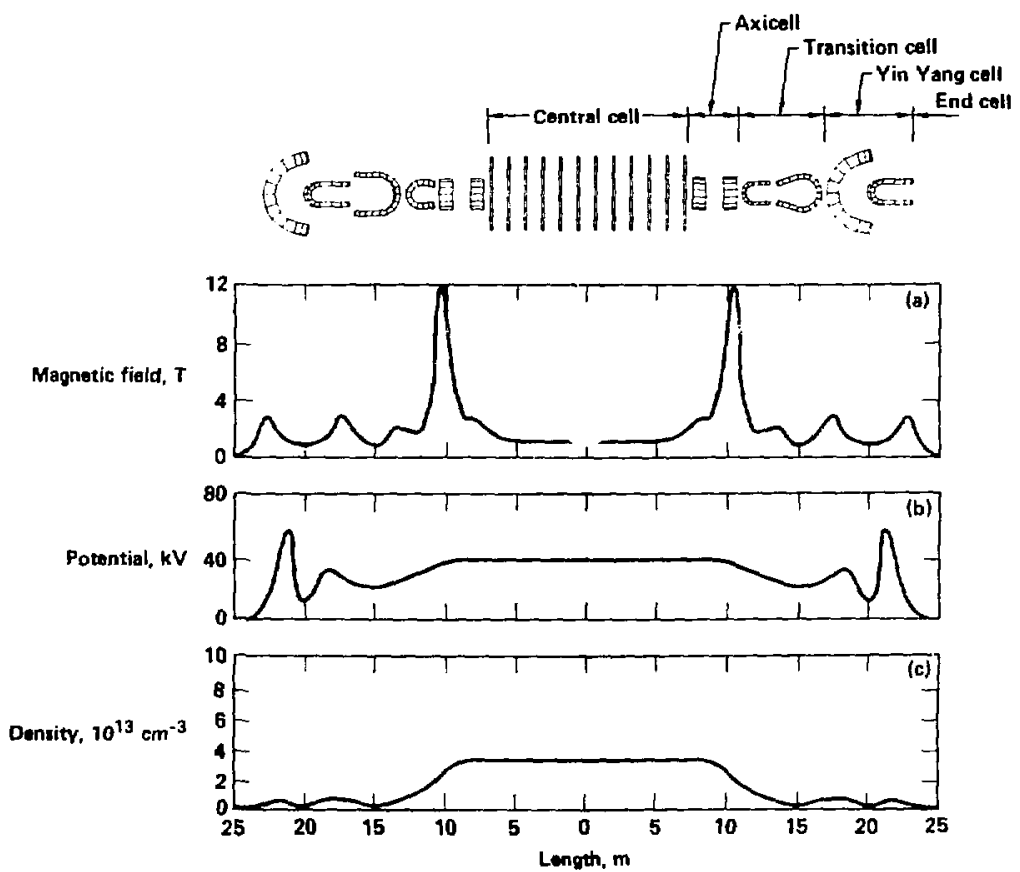

Flgure 1.6 Vacuum magnetic field (A), axial plasma potential (B), and plasma density (C) for a tandem mirror with thermal barriers 
axisymmetric center cell separated from an MHD anchor and includes electron lsolation from the end plugs by a thermal barrier.

\subsection{Physics Issues of Alpha Conf Inement in Tandem Mirror Reactors}

Tandem mirror reactors are expected to operate in steady state with the center cell ignited by the $3.52 \mathrm{MeV}$ alpha particle heating. We are therefore concerned with all aspects of the the lifetime of the alpha particles in the tandem mirror reactor from birth through slowing down to ultimate loss from the system. The 1ssues involved are extremely complicated and the purpose of this thesis is to ideriti? the more important ones and to study their role in determining the 1 gnition condition. Specifically, we are concerned with modeling the distribution of the alpha particle source function in the equilibrium plasma, the thermalized alpha density, and the alpha energy deposition profile. The profiles are determined by the plasma condition along the alpha orbits in the specific confinement geometry. In this section, we shall discuss qualitatively the physics issues with the alpha birth and thermalization process.

The alpha particles are created by the fusion of deuterlum and tritium ions in the plasma. The alpha particle source distribution function, therefore, is determined by the distribution of deuterium and tritium particles in the plasma. Therefore, one of the issues to be addressed in this thesis is the proper representation of the alphas particle source function in generis tandem mirror reactors. Through momentum balance considerationa, the alpha particle will be enitted with an average energy of $3.52 \mathrm{MeV}$. Because the alpha 
particles are doubly sharged, their motions will be affected by the electric and magnetic flelds present. The electric and magnetic fields arise from both externally imposed flelds, such as the vacuum magnetic field and self-conslstent macroscopic flelds, such as due to the plasma dlamagnetism and ambipolar potential, as well as microscopic fields associated with the individual charged particles. The vacuum magnetic field is readily computed from specification of the confinement coils. The distribution of the plasma pressure confined by the externaliy impused field is determined from solving the steady state MHD equation for momenturr balanoe,

$$
\nabla p_{1}=\bar{j} \times \bar{B}_{h}
$$

where the current density 1 s given by Ampere's Law,

$$
\nabla \times \vec{B}_{V}=\mu_{0} \vec{J}
$$

Comblning the two equations, we obtain

$$
\nabla\left(p_{1}+\frac{B_{v}^{2}}{2 \mu_{0}}\right)=\frac{1}{\mu_{0}}\left(\bar{B}_{v} \cdot \nabla\right) \bar{B}_{v}
$$

where $p_{\perp}$ is the plasma pressure perpendicular to the local confinement magnetic field. The right side of Eq. (1.3.3) is the curvature which Is exactly zero In a straight and uniform solenoldal magnetic field. 
In actual magnetic field configurations, the approximation of setting the right side equal to zero, whlch $1 \mathrm{~s}$ commonly known in mirror confinement theory as the "long, thin approximation," ylelds for the solution of the self-consistent magnetic fleld,

$$
B_{a}=B_{v}\left(1-\frac{2 \mu_{0} P_{1}}{B_{v}^{2}}\right)^{1 / 2} .
$$

The effectiveness in the utilization of the vacuum magnetic fieid is of ten characterized by the parameter, B, defined as

$$
B=\frac{2 \mu_{o} P_{i}}{B_{v}^{2}} \text {, }
$$

which is the ratio of the plasma pressure to the vacuum magnetic field pressure. A characteristic of magnetic mirror confinement, which makes such confinement attractive, is that $\beta$-values approaching unity are possible. When beta approaches unity, the magnetic field inside the plasma goes to zero and, as a result, the Larmour radius, Biven by

$$
\rho=\frac{m v_{\perp}}{q B_{a}},
$$

of the very energetic alpha particles can become comparabile or even larger than the plasma radius. Therefore, plasma diamagnetism can have an important effect on alpha particle confinement. 
As we have noted, the tandem mirror concept depends on the ambipolar potential associated with magnetic mirror confinement to plug the end losses. The presence of an ambipolar potential implies that there is a radial electric field which is associated with ion and electron radial density gradients. In the presence of a radial electric and axial magnetic field, the motion of the charged particles can be decomposed into three parts, a cyclotron gyration about the gidding center along a fleld line, motion of the guiding center along the fleld line, and a transyerse drift perpendicular to both the electric and magnetic fieid, with velocity given by

$$
\bar{V}_{D}=\frac{\vec{E} \times \bar{B}}{B^{2}} .
$$

Therefore, in order to properly account for the motion of the alpha particles in the tandem mirror system, we need to also take into account the axial and radial electric fields.

The motion of the alpha particles is affected not only by the macroscopic but also the microscopic fields associated with individual particles, The Individual charged particles interact through long range Coulumb forces. In a dense plasma, each paricicle experiences the fields associated with many other particles in its vicinity simultaneously. As a result, the interaction is in the form of small perturbations to its thermal velocity and direction of motion. The effect of thls type of "collisional" interaction on a particle 
distribution, $f$, is described by the Fokker Planck collision operator, 30,31

$\left.\frac{\partial f}{\partial t}\right|_{c}=-\frac{\partial\left\langle\Delta w_{H}\right\rangle f}{\partial w}+\frac{\partial^{2}\left[0.5\left\langle\left(\Delta w_{\|}\right)^{2}\right\rangle f\right]}{\partial w^{2}}+\frac{\partial^{2}\left[0.5\left\langle\left(\Delta w_{\perp}\right)^{2}\right\rangle f\right]}{\partial w^{2}}+0\left(\Delta w^{3}\right)$,

where $w$ is the velocity and $w_{f}$ and $w_{1}$ denote the parallel and perpendicular components, respectively. The quantity $\Delta \mathrm{w}$ represents changes to the velocity as a result of the interactions. The brackets represent averages over the distribution of field particles in the vicinity of the test particle. Spitzer ${ }^{32}$ has physically interpreted the quantities $\left\langle\Delta w_{\|}\right\rangle,\left\langle\left(\Delta w_{\|}\right)^{2}\right\rangle$ and $\left\langle\left(\Delta w_{1}\right)^{2}\right\rangle$ as dynamical friction, velocity dispersion, and pitch angle scatterlng, respectively. He was aiso able to derive explicit expressions for these quantities on the basis of a test particle interacting with a background plasma, characterized by its ionlc species, temperature, and density. Making use of these quantities, it is possible :o define the following characterist 1c times:

$$
\tau_{D}=\frac{w}{\left\langle\Delta w_{\|}\right\rangle^{2}}
$$

for drag time,

$$
\tau_{\varepsilon}=\frac{w^{2}}{4\left\langle\left(\Delta w_{\|}\right)^{2}\right\rangle}
$$


for energy exchange time (thermalization time), and

$$
\tau_{1}=\frac{w^{2}}{\left\langle\left(\Delta w_{1}\right)^{2}\right\rangle}
$$

for the cumulative $90^{\circ}$ pitch-angle scattering time.

The confinement and thermalization of the alpha in a tandem

mirror reactor is a function of its velocity and radial position, along with the plasma properties at this position. Alphas are born isotropically at $3.52 \mathrm{MeV}$, which will be much greater than any confinement potential and if they are born in the loss cone, they will be lost immediately. Those that are born outside the loss cone will be confined initially and may be lost as they are dragged down in energy and pitch angle scatter on the background plasma. Alphas that scatter into the loss cone with energies greater than $\Phi_{\mathrm{pc}}$ will also be lost. In order to effectively utilize the alpha particles for sustaining the fusion plasma, the system must be designed such that the alpha particle confinement time is at least comparable with the drag tine.

Once the alpha particles are thermalized, their average energy will be lower than the plugging potential, and they will be electrostaticaliy confined. Furthernore, because they are doubly charged, they experlence a potential barrler that is twice as high as for the hydrogenic lons. As a result, thermalized alphas will tend to accumulate in che burning plasma and displace the density of the reacting fuel. If suah acoumulation is not controlled, there will eventually be insufficient fuel to generate the fusion power needed to 
sustain the plasma and the reaction wlll quench. Therefore, in addition to confinement and thermalization, removal of the alpha particles is also an important issue in the maintenance of an ignited fusion plasma.

Finally, we should also note that the reacting plasma is expected to be radially inhomogeneous in both density and temperature. Because the alpha particles are initially very energetic, their gyroradius will be large, particularly in a high beta plasma, and they will traverse regions of very different plasma density and temperature in each gyration. Since the drag rate depends on the local density and temperature, there is an uneven slowing down rate along each gyro orbit. is the alpha loses energy, its gyroradlus becomes correspondingly smaller. It can readily be demonstrated from physical considerations that this uneven drag rate has the effect of pulling the alpha guiding center towards the region of high drag, i.e. regions of high density and low temperature. In general, for motion of a charged particle in a force field, $\bar{F}$ transverse to a magnetic field, there is a drift of the guiding center with velocity,

$$
\bar{V}_{D}=\frac{\bar{E} \times \bar{B}}{B^{2}} .
$$

In the case of present interest, the net force, $\bar{F}$, is the difference of the unequal azimuthal drag rates across the gyro orbit of the particle. For a cylindrical plasma with radially decreasing temperature and density profiles, typical of a hot reacting thermonuclear plasma 
surrounded by a cool halo plasma, the resulting drift is radially outward. The effect of radial drag gradients, therefore, is to move high energy particles in the direction of the gradient. If the drift velocity is sufficiently high, core heating will be reduced and the alpha particles will deposit a significant fraction of their energy in the halo, whlch is generally poorly confined. This effect can also prevent the attalnuent of Ignition condition and must be properly considered.

\subsection{Methods of Analysis}

The rigorois approach to determining the energy deposition of the alpha particles in the fusion plasma is to solve the Boltzmann equation for the al oha particle distribution, $f_{\alpha}$, in the sixdimensional phase space,

$$
\frac{\partial f_{\alpha}}{\partial t}+\bar{v} \cdot \frac{\partial f_{\alpha}}{\partial \bar{r}}+\frac{\bar{F}}{m} \cdot \frac{\partial f_{\alpha}}{\partial \bar{v}}=\left.\frac{\partial f_{\alpha}}{\partial t}\right|_{c}+s_{\alpha}+L_{\alpha},
$$

where $S_{\alpha}$ and $L_{\alpha}$ are the alpha particle source and sink terms in real space and $\left.\frac{\partial f_{\alpha}}{\partial t}\right|_{c}$ is the Fokker-Planck operator for Coulomb collisions which may be interpreted as a source or sink in velocity space. The left side of the equation is just the total time derlvative of the distribution function.

There are many difflculties that prevent the general analytical solution of Eq. $(1.4 .1)$. One problem is that this equation is 
nonlinear. However, we expect the denslty of the alpha particles to be small compared to the density of the reacting plasma, and it is reasonable to only consider the alpha interaction with the distribution of the plasma electrons and lons. Therefore, we only need to be concerned with the solution of the linearized form of Eq. (1.4.1). There are still other difficulties. First of all, the disiribution of plasma electrons and ions, which is determined by the fully nonlinear Boltzmann equation, is not known. Therefore, suitable approximations must be assumed to represent them. Secondly, the problem is multidimensional; for any realistic representation of a tandem mirror system, we generally need to conslder at least one spatial (radial) and two velocity (parallel and perpendicular to the magnetic field) directions. It is extremely unlikely that analytical solutions can be found for specific confinement field conf1gurations. In general, therefore, it is necessary to resort to computer solutions by numerical methods.

We note that the left side of Eq. (1.4.1) describes the phase space trajectory of panticles in the distribution, while the right side accounts for source and sink terms in both physical and velocity space due to colisions. In considering the numerisal solution of the Boltzmann equation, there are several characteristic times of interest. The first is the cyciotron gyration time,

$$
\tau_{c}=\frac{1}{2 \pi \omega_{c}}=\frac{1}{2 \pi} \quad \frac{m}{q^{B}} .
$$


The second characteristic time is associated with the axial bounce period of particles as they are reflected from end to end in a mirror trap of length, $L$,

$$
\tau_{b}=\frac{L}{|\bar{v}|} .
$$

There is also a characteristic time associated with the azimuthal drift period,

$$
T_{\phi}=\frac{2 \pi r_{p}}{\left|\bar{v}_{d}\right|} .
$$

Finally we have the characterlstic times associated with collisionai Interactions of the alpha particles with the bulk plasma, i.e. the drag, dispersion, and pitch-angle scattering times. In the case of $3.52 \mathrm{MeV}$ alpha particles in a magnetically confined plasma, these characteristic times are ordered as follows:

$$
\tau_{c} \ll \tau_{b}-\tau_{\phi} \ll \tau_{d} \ll \tau_{\perp} \ll \tau_{e},
$$

namely, the characteristic times associated with the terms on the right slde of Eq. (1.4.1) are Jong compared to the characteristic times for periodic motion in phase space associated with terms on the left side of the equation. However, it is precisely these slow collisional processes that cause the alpha particles to thermalize and be 
transported in phase space. Therefore, although these terms on the right side of the equation are much langer in magnitude compared to those on the left, they can not be ignored, since they govern the relaxation process. On the other hand, it is also evident that collisional relaxation is not a localized process and must be calculated by averaging over the phase space trajectory of the particles; these trajectorles are determined by $\tau_{c}$ ' $\tau_{b}$, and $\tau_{\phi}$. The method we have chosen to use in studying alpha particle thermalization is the Monte-Carlo technique, which constructs a solution based on random sampling. The technique has the advantage that the particle trajectory in phase space can be followed in detail. Furthermore, because the cyclotron gyration feriod is very short, compared to characteristic times for any other processes of interest, and the gyroradius 15 unlquely determined by $\mu$, we need only to follow the motion of the guiding centers. The effects of the infrequent collisions are then evaluated by sampling from appropriate probability distributions. To evaluate the displacement of the guiding center due to collisions, it is necessary to know the exact position of the particle in relation to the gulding center. We can recover this information statiscically by sampling. A significant portion of this thesis is concerned with developing reasonable models for representation of the system in a process that is computaticrally fast. The solution is then constructed from the results of a large number of samples. The accuracy of the solution $1 \mathrm{~s}$ determined primarily by the number of alphas in the sample. In this thesis, the algorithm is then 
applied to carry out parametric studies of alpha particle slowdown in a generic tandem mirror reactor.

The center-cell plasma parameters for a generic tanden mirror fusion reactor that have been used in this study are chosen to be consistent with present reactor designs. A comparison of the generic parameters with those of two current reactor design studies is made in Table 1.1.

\section{TABLE 1.1}

COMPARISON OE THE GENERIC TANDEM MIRROR REACTOR PARAMETERS WITH THE SUPER-TARA AND OCTOPOLE END CELL DESIGNS.

\begin{tabular}{|c|c|c|c|c|}
\hline Parameter & Super-Tara $^{33}$ & Octopole & End $\mathrm{Cell}^{34}$ & Generic Design \\
\hline$P(M W)$ & 1000 & 500 & 1000 & 1000 \\
\hline$r_{p}(m)$ & 0.51 & 0.45 & 0.45 & 0.5 \\
\hline$L_{c}(m)$ & 24 & 50 & 100 & 60 \\
\hline $\mathrm{B}_{\mathrm{c}}(\mathrm{T})$ & 3.5 & 3.3 & 3.3 & 3.0 \\
\hline$B_{m}(T)$ & 25 & 24 & 24 & 24 \\
\hline B (Peak Beta) & 0.84 & 0.75 & 0.75 & 0.8 \\
\hline$T_{1}(\mathrm{keV})$ & 40 & 40 & 40 & 40 \\
\hline$T_{e}(k e V)$ & 28 & 27 & 27 & 27 \\
\hline $\mathrm{n}_{1}-10^{14} \mathrm{~cm}^{-3}$ & 3.01 & 2.3 & 2.3 & 1.65 \\
\hline
\end{tabular}

The details of the numerlcal simliation and the computed results are presented in the following chapters. The specific magnetic and electrostatio fleld models are descrlbed in Chapter 2. The guiding center equations of motion for the alpha particles are developed in 
Chapter 3. A detalled description of the Monte-Carlo scheme for treating collisional effects is presented in Chapter 4. The varlous types of density and temperature models for representing the bulk plasma jysten used in the study is described in Chapter 5. The results of the study and the various diagnostles used to verlfy the validity of the calculational technique are presented in Chapter 6 . General conclusions from this study and suggestions for future research are given In Chapter $T$. 


\section{PART 2}

\section{MAGNETIC AND ELECTRIC FIELDS}

\subsection{Introduction}

An essential first step in studying alpha particle thermalization in magnetically confined fusion plasmas is to develop a method for accurately calculating the confining magnetic fields and electrostatic potentials. An exact analysis begins with a description of the vacuum magnetic fields. Next, the plasma diamagnetic effects and local fluctuations in the magnetic fleld would be generated by a self-consigtent degcription of the confined plasma. The radial and axial electrostatic potentials would also be determined selfconsistently. The development of a self-consistent fleld and potential model requires a major computational effort. Such a technique, although desirable, is not yet available for determining the magnetic confinement fields and electrostatic potentials that are associated with alpha particle thermalization in tandem mirrors. Even though a self-consistent calculation is unavailable, it is possible to determine the vacuum magnetic fields along with the plasma diamagnetic effects and the axial potentlal by using physically reasonable and computationally quick models. The radial potential which is generited by the plasma radial density and temperatire profile is modeled based on radial potential control techniques ${ }^{35}$ that are being tested on the TMX-U experiment. The vacuum magnetic fleld models along with the plasma diamagnetic and electrostatic potential models used in this study are presented in the following sections. 


\subsection{Tandem Mirror Vacuum Magnetic Fleldg}

Vacuum magnetic fields in the plasma can be found by numerically integrating Ampere's Law over defined current elements. There are many 3-D electromagnetic codes, e.g. EFFI, ${ }^{36}$ that can be used to calculate the vacuum flelds from defined current elements. In using Monte-Carlo techniques to follow particle trajectories, the magnetic fleld is needed at every calculated particle position, and determining vacuum field values from three-dimensional codes would require an excessive amount of computer time. Analytical methads which approximate the magnetic fields can be used, but the derivative of the fleld, which is needed for particle drift calculations, is not smooth. We combine field values calculated at fixed nodal points from EFFI with the paraxial approximation, ${ }^{37}$ given to order $r^{2}$ by

$$
\begin{aligned}
& B_{x}=-\frac{x}{2}(f(z)-g(z)) \\
& B_{y}=-\frac{y}{2}(f(z)+g(z)) \\
& B_{z}=f(z)
\end{aligned}
$$

to calculate the magnetic fields in the plasma. The value of $f(z)$ is found from EFFI calculations of $B_{z}$ at points along the plasma axis. The function of $\mathrm{g}(\mathrm{z})$ is determined from 


$$
B(z)=\eta^{\prime}(z) f(x) \text {. }
$$

where $\eta^{\prime}(z)$ is the derivative of the magnetic flux tube ellipticity. The ellipticity is found from the conservation of magnetic flux given by

$$
\begin{aligned}
\psi_{\phi} & =r^{2} f(0) \\
& =f(z) x^{2} \exp (-n(z))+f(z) y^{2} \exp (n(z)),
\end{aligned}
$$

where $\psi_{\phi}$ is the magnetic flux. When y equals zero, the ellipticity is

$$
\pi(z)=\operatorname{Ln} \frac{x^{2} f(z)}{r^{2} f(0)},
$$

with the derivative along $z$ given as:

$$
\eta^{\prime}(z)=\frac{r^{2} f(0)}{x^{2} f(z)} f^{\prime}(z) .
$$

Values of $n^{\prime}(z)$ are determined from EFFI calculations of $B_{z}$ fon a given $x$-displacement at points along the $z$ axis. We assume that the ellipticity for a flux tube at arbitrary $z$ is constant in the radial direction, and therefore we can $\mathrm{find}_{\mathrm{x}}, \mathrm{B}_{y}$, and $\mathrm{B}_{z}$ at any point in the plasma. Values of $f^{\prime}(z), f^{\prime}(z)$, and $n(z)$ can be found at any location 
by using a cublc spline technique between nodal points. The cubic spline was incorporated into the calculation by ohnishi and $1 \mathrm{~s}$ described in Ref. 19.

The paraxial approxination can also be used to relate plasma parameters along a flux tube to the parameters at the plasma midplane. For example, the density and temperature are assumed to be functions of the flux coordinate $\left(\psi_{\phi}\right)$ and the radius. These variables are constant along a flux tube and are given by

$$
\begin{aligned}
& n\left(\psi_{\phi}, r\right)=n\left(\psi_{\phi}(z=0), r(z=0)\right), \\
& T\left(\psi_{\phi}, r\right)=T\left(\psi_{\phi}(z=0), r(z=0)\right) .
\end{aligned}
$$

The radial plasma potential $\left(\phi_{r}\right)$ is a function of the radial density and temperature profiles, and it may also be determined at any $z$ by assuming that $\phi_{r}$ is a function of $\psi_{\phi}$ and $r$.

$$
\phi_{r}\left(\psi_{\phi}, r\right)=\phi_{r}\left(\psi_{\phi}(z=0), r(z=0)\right) .
$$

Therefore, once the plasma density, temperature, and radial potential profiles are defined at any $z$ loctiction they can be determined at any other plasma location.

The calculation of the magnetic fields, plasma temperature, densities, and radial potentials can be further simplified for alphas 
In axisymmetrle fields and for a class of alpha guiding center trajectories, called "nonresonant" trajectories, 28 in nonaxisymmetric tandem .21 rrors. The bounce motion of these alphas can be neglected and their transport can be simulated at the plasma midplane. This simplifies the description of the vacuum fields, since only the midplane values are required. However, when the bounce motion cannot be neglected, the techniques described above nust be used. Although the Monte-Carlo technique can perform detailed guiding center trajectory calculations throughout the plasma, they are not needed for the present axisymmetric reactor designs.

Particle motion in the central cell of an axisymetric mirror can be described by knowing the density, temperature, and magnetic field at the plasma midplane. This is true because the curvature drifts generated in the axisymetric mirrors of the center cell are azimuthal. Furthermore, this drift is generally small compared to $\bar{E} \times \bar{B}$ and grad $B$ drifts, and since they do not affect alpha transport in axisymmetric tandem mirrors, the curvature drift can be neglected. By neglecting the curvature drifts, only the vacuum magnetic fields at the plasma midplane are required, and these are given by

$$
\begin{aligned}
& B_{x}=B_{y}=0, \\
& B_{z}=|\bar{B}| .
\end{aligned}
$$

These vacuum flelds also satisfy the following conditions, 


$$
\nabla \cdot \overline{\mathrm{B}}=0
$$

$$
\nabla \times \bar{B}=0
$$

The vacuum magnetic field is altered by the plasma pressure, as shown by Eq. $(1 \cdot 3.4)$. Because there is a radial pressure profile, there is also a radial gradient in 8 . The long thin approximation for the magnetic field at the plasma midplane is dependent on the radial position, i.e.,

$$
B_{a}=B_{v}[1-B(r)]^{\frac{1}{2}} \text {. }
$$

We define the pressure profile in terms of the density and temperature scale lengths $r_{p D}$ and $r_{p T}$ as

$$
\begin{aligned}
& n=n_{0} f\left(\frac{r}{r_{p D}}\right), \\
& T=T_{0} f\left(\frac{r}{r_{p T}}\right),
\end{aligned}
$$

where the subseript "o" denotes the value at $r=0$.

The pressure is given as

$$
p_{0}=n_{o i}^{k T} \text { oi }+n_{o e} k T_{o e}
$$


where the subscripts "i" and "e" refer to fons and electrons respectively. In a quasineutral plasma, the electron and ion densities are essentially equal throughout the plasma, therefore,

$$
n_{i}-n_{e} .
$$

The ion and electron temperatures can be different so the plasma pressure becomes

$$
p=n_{e o} k\left(T_{o i}+T_{o \rho}\right) f\left(\frac{r}{r_{p D}}\right) f\left(\frac{r}{r_{p T}}\right)
$$

or

$$
p=p_{0} F\left(\frac{r}{r_{p D}}\right) f\left(\frac{r}{r_{p T}}\right) .
$$

where we have defined $\mathrm{p}_{0}=\mathrm{n}_{\mathrm{eo}} \mathrm{k}\left(\mathrm{T}_{10}+\mathrm{T}\right.$ eo $)$. Implicit in this relation is the assumption that the radial density profiles for ions and electrons are equal and the temperature profiles are assumed to have the same radial dependence. The radial gradient of $B$, which is needed to determine alpha drift motion, is

$$
\nabla B_{a}=\nabla\left\{B_{V}\left[1-B_{o} f\left(\frac{r}{r D}\right) f\left(\frac{r}{r T}\right)\right]^{\frac{1}{2}}\right\},
$$

and this simplifles to 


$$
\nabla B_{a}=-\frac{B_{0}}{B_{v}(1-8(r))^{1 / 2}} \nabla\left(f\left(\frac{r}{r_{p D}}\right) f\left(\frac{r}{r_{p T}}\right)\right) .
$$

\subsection{Diamagnetic Effects on Magnetic Fields}

Plasma diamagnetic effects as shown above lower the magnituce of the vacuum magnetic field. The diamagnetic effect $1 \mathrm{~s}$ greatest in the tandem mirror wenter cell, where $B$ is high, and small at the inirror throat where $B$ is low. This effect creates magnetic wells that are deeper $t_{1}$ an the designed vacuum wells. AIthough the diamagnetic effect reduces the magnitude of $B, 1$ t has a negligible effect on fleld line curvature, as discussed in Ref. 38 . Therefore, at any point in the plasma, the direction of the magnetic field is given by the vacuum field unit vectors at that point.

Diamagnetism also affects the adiabaticity conditions of the plasma. Conn, et al. ${ }^{39}$ have studied the conditions in which $\mu$ is an adiabatic constant in high $\beta$ mirror machines. Confined particles are absolutely adiabatic when $\rho / L$, where $L$ is the smallest magnetic scale length, is less than 0.1 . In a high $B$ plasma, the $\alpha$ Larmor radius can approach values of the magnetic scale length, so that the value of $\mathrm{p} / \mathrm{L}$ can approach unity. Even when $\rho / L$ approaches unity, the magnetic field can be deslgned to ensure conservation of $\mu$. We have assumed that the axial variation of $\beta$ is made smooth, so the a particles are adiabatic. Radial temperature and density profiles are made so that $\rho / r_{p}<1$, such that the radial gradients do not affect the adiabaticity. 


\subsection{Axlal and Radial Potent1al Profiles}

Axial and radial potential profiles are modeled consistent with present reactor designs. The radial potential can be modeled using Eq. (2.2.6), Dut from radial transport considerations, it is desirable to have this potential at ground by using radial potential control plates. 35 Therefore, we set the radial potential to zero, and there is no $\vec{E} \times \overline{\mathrm{B}}$ drift for the alpha particles. Elimination of the radial potential preverts the plasma ions from having large azimuthal drifts in nonsymmetric tandems to minimize plasma ion radial transport. Energetic alphas, however, have large azimuthal dlamagnetic drifts which are in the opposite direction from the $\overline{\mathrm{E}} \times \overline{\mathrm{B}}$ drifts. Therefore, alphas in nonaxisymmetric $h i g h-\beta$ tandems which have no radial potential can still have resonant particle diffusion. Resonant diffusion of alphas is prevented by requiring the center cell fields to be fully axisymmetric. The radial potential in axisymmetric tandems affects only the alpha azimuthal motion and does not affect alpha transport. Alpha particles that are scattered into the loss cone with energies less than the confining potential are mirror trapped, but energetic al whas will pass right over the potentlal peak. Potential peak values are approximately $4 \mathrm{~T}_{1}$, so that alphas with a plus two charge must have energles less than approximately $8 \mathrm{~T}_{\mathrm{i}}$ or approximately $500 \mathrm{keV}$, which means that less than $15 \%$ of alpha energy is potentially confined. The majority of this $500 \mathrm{keV}$ will be deposited in the center cell region, since the density in the transition and anchor regions is lower than the center cell density. Because of these considerations, 
the axlal potential is modeled as a square well with the axial peaks occuring at the center cell mirror peaks (see Fig. 2.1). The magnitude of the axial potential is given by $k \cdot T_{1}$ where $k$ is an input parameter chosen as 4 for this study. The radial shape of the axial plugging potential follows $T_{1}$. 

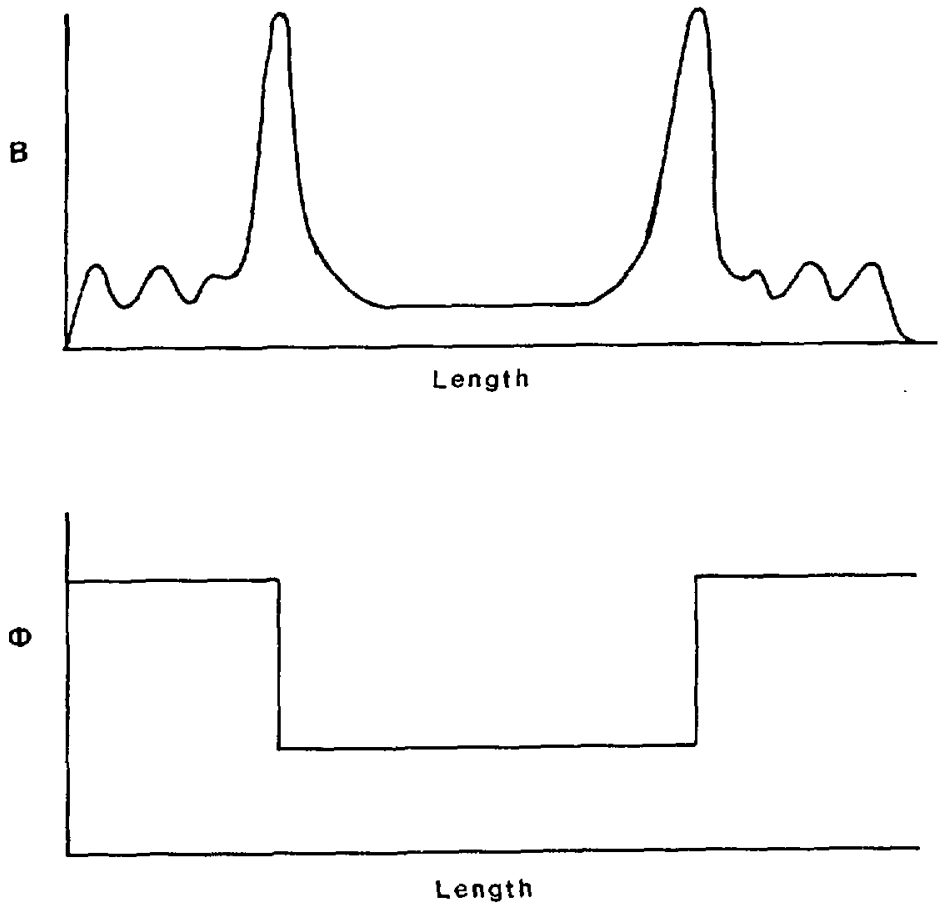

Flgure 2.1 Vacium magnetio fleld (A), and axial plasma potential (B) for the generic reactor design 


\section{PART 3}

\section{GUIDING CENTER THEORY}

\section{$3.1 \quad$ Introduction}

In general, alpha particle trajectories in a tandem mirror reactor can be described as a fast gyro motion, coupled with a drift perpendicular to the background magnetic field, and an acceleration parallel to the magnetic field. The alpha fluid motion is a part of the Boltzman equation, given by Eq. (1.4.1), and an individual alpha trajectory in velocity space can be found when $\mathrm{f}_{\alpha}$ is defined as a single particle distribution function. The left side of Eq. (1.4.1), when multiplied by $\bar{v}$ and integrated over velocity space for this single particle distribution, becomes

$$
\frac{\mathrm{d} \bar{v}_{a}}{\mathrm{~d} t}=\frac{\mathrm{q}_{\alpha} \mathrm{B}}{\mathrm{m}_{\alpha}}\left(\frac{\bar{E}}{\mathrm{~B}}+\overline{\mathrm{v}}_{\alpha} \times \frac{\overline{\mathrm{B}}}{\mathrm{B}}\right)
$$

where $\vec{v}$ is the actual particle velocity. This is the alpha equation of motion as given by Eq. $(1.1 .1)$.

The right side of the Boltzman equation contalns the collision

operator $\left.\frac{\partial f_{a}}{\partial t}\right|_{c}$ plus source and sink terms. Part of the collision

operator is the dynamical friction or drag which continuously slows the high energy alphas down to lower energles without affecting the alpha pitch angle. Because the drag is continuous, it can be treated in a similar manner as the electromagnetlc forces during the slowing down process. Even though drag is continuous, it can be simulated by a 
discrete collision in which accumulated drag eflects shrink the orbit and the drag gradients cause radial gulding center drifts.

The alpha motion calculated in a Monte-Carlo technique must be effectively simulated by sampling the collision process many times and by following the alpha between collision sampling events. The alpha mean free path between sampling events is a fraction of $\tau_{D}|\bar{v}|$, which is approximately $10^{5}$ to $10^{7} \mathrm{~m}$, and $1 \mathrm{t}$ is quite complicated. Even a fraction of this mean free path is long, and if it were computed exactly, it would require an excessive amount of computer time. Fortunately, $\mu$ is an adiabatic invarlant between collisions, so the alpha guiding center can be followed and, in axially unfform and axisymmetric reactors, the bounce motion can be neglected, so that only the alpha drift motion at the reactor midplane needs to be followed. The guiding center trajectories derived from Eq. (3.1.1) are presented in the next section, followed by a discussion of the radial drag drifts.

\subsection{Classical Gufding Center Theory}

A charged particle in a uniform magnetic fleld gyrates about a magnetic fleld line as if 1 t were attached to the line by a string. The point where the imaginary string attaches to the fleld line is the guiding center. The guiding center drifts off the tield line shen there are transverse electric fields, magnetlo and plasma pressure gradients, and magnetic-field line curvature. These complex Lrajectorlas can be almplifled by integrating over the fast gyro motion and collowing the guiding center motion. 
The concept of guiding center motion can be used when the scale lengths for fleld changes are small over a Larmor orbit. This scale factor $\left(\frac{p}{L}\right)$ can be found by non-dimensionalizing $\mathrm{Eq} \cdot(3.1 .1)$, given by

$$
\frac{\rho}{\mathrm{L}} \frac{\overline{d v}}{d T}=\frac{\vec{E}}{v_{0} B_{0}}+\vec{v} \times \tilde{B}
$$

where

$$
\begin{aligned}
& \tilde{v}=\frac{\bar{v}}{v_{0}}, \\
& T=\frac{L}{v_{0} t}, \\
& \tilde{E}=\frac{E}{E_{0}}, \\
& \tilde{B}=\frac{B}{B_{0}},
\end{aligned}
$$

The values of $E_{o}, B_{o}$, and $v_{o}$ represent typlcal values for the electric and magnetic fields and particle speed. When $\frac{\rho}{L}$ is small, the solution of Eq. (3.2.1) can be found by expanding the electric and magnetic fields in terins of this scale factor. This is an essential first step in deriving the guiding center equations of motion.

A formal derivation of the guiding center equations has been discussed by Northrop, ${ }^{40}$ and a brief review of his results will be presented here. First, the radius vector $(\bar{r})$ is defined by a guiding center component $(\bar{R})$ and the Larmor radius $(\bar{\rho})$,

$$
\bar{r}=\bar{R}+\bar{\rho}
$$


We use an orthogonal coordinate system with $\hat{e}_{1}$ equal to $\bar{B} / B, \hat{e}_{2}$ is perpendicular to $\hat{e}_{1}$, which defines $\hat{e}_{3}$ as $\hat{e}_{1} \times \hat{e}_{2}$. In uniform rields we could substitute Eq. $(3.2 .2)$ Into Eq. (3.1.1) and evaluate the fields at either $\bar{r}$ or $\bar{R}$. In the guiding center approximation we approximate the flelds at $\bar{r}$ by expanding the fields at $\overline{\mathrm{R}}$ using the expansion parameter $\delta=\frac{\rho}{\mathrm{L}}$. Using this technique, the acceleration $\ddot{\bar{r}}=\ddot{\overline{\mathrm{R}}}+\ddot{\bar{\rho}}$ can be found to order $\delta$. The resulting equation can be averaged over a Larmor radius, neglecting terms of order $\delta^{2}$ or higher. The guiding center parallel and drift motion in the steady-state magnetic and potential fields of a tandem mirror are then glven by

$$
\begin{gathered}
\frac{d v_{\|}}{d t}=-\frac{\mu_{a}}{m} \hat{e}_{1} \cdot \nabla B_{a}+\frac{q}{m} E_{\|}, \\
\bar{v}_{D}=-\frac{\bar{B} \times \bar{E}}{B}+\frac{\mu_{a}}{q \bar{B}_{a}} \hat{e}_{1} \times \nabla_{a}+\frac{m v_{\|}^{2}}{q_{a}} \hat{e}_{1} \times\left(\hat{e}_{1} \cdot \nabla\right) \hat{e},
\end{gathered}
$$

The terms on the right side of Eq. (3.2.4) represent the drift motion caused by transverse electric flelds, magnetic fleld gradients, and magnetic curvature, respectively. Implicit in these equations is the assumption that gravitational ef fects are negligible and the electric field parallel to $B$ is of order $\delta$.

\subsection{Classical Drift Simulation}

In a uniform plasma, the slmulation of alpha guiding center motion depends on the plasma electrostatic potentials and the confining 
magnetic flelds. When the confining magnetic flelds are nonaxisymmetric, some of the confined particles exhibit large, perlodic, radial drift motions. Ryutov and stupakov ${ }^{28}$ were the first to show that particles in a nonaxisymmetric tandem mirror with azimuthal drifts per bounce equal to $\frac{n \pi}{2}$ when $n$ is $1,3,5, \ldots$. exhibit large radial resonant excursions which enhance radial transport. It can be shown that the resonant width is finite in $\mu$ and particles near resonant conditions also have large radial excursions. Therefore, the parallel and drift motion of resonant or near resonant alphas trapped in a nonaxisymmetric tandem mirror must be followed between collision sampling events. Alphas that are far from resonant conditions have azimuthal drift motions that can be approximated by axis-encircling guiding-center trajectorles whose bounce motion can be neglected. This is also true for alphas trapped in axisymmetric tandem fields; therefore, alphas with axis-encircling guiding-center trajectories are followed at the plasma midplane. Because mirrorconfinement magnetlo flelds are still evolving, we have includer both trajectory following schemes in this simulation.

Alphe particles in a nonsymmetric tandem mirror that are resonant or near resonant must be followed by integrating Eq. (3.2.3) and solving Eq. (3.2.4). This simulation is computed in the center cell, where the nonaxisymmetric fleld effects are negligible, by rotating the particle around the axis based on the drift veloolty from Eq. (3.2.4) for the approprlate center cell length. When the particle enters reglons where nonaxisymmetric flelds affect the radial drift 
motion, then the full solution of Eq. (3.2.3) and (3.2.4) is required. One method for solving these equations is to use conservation of energy to find $v_{\|}$and Eq. $(3.2 .4)$ to find $\bar{v}_{D}$. This technique has been used in a leap-frog predictor-corrector soheme in which the guiding center velocity is known at time $t_{1}$ and calculated at $t_{1+2}$, using the guiding center position and fleld information calculated at $t_{1+1} \cdot 41,42 \mathrm{Tr} . \mathrm{d}$ algorithm has been used to track ions in a tokamak reactor, but it does not accurately track all trajectories through a mirror bounce because the direction of $v_{\|}$is lost when conservation of energy is used. The direction of $v_{\|}$must be found by integrating Eq. (3.2.3). Therefore, the gulding center algorithm that has been used is a leap-frog predictor-corrector scheme that solves Eq. (3.2.3) by Euler Integration, calculates $\bar{v}_{p}$ from $(3.2 .4)$, and determines the guiding ceriter position by Euler integration of the parallel and drift valocitles. This new algorlthm is also accurate and tracks particles through a mirror bounce with one predictor and corrector step. Plots of alpha trajectories determined by this algorlthm are given in Ref's. 19 and 20 .

Particles that are nonresonant in nonaxisymmetric tandem mirrors, or all particles in an axisynimetrio tandem, can be followed at the plasma mldplane. By neglecting the bounce motion, the paraliel motion is ellininated so that only $\bar{v}_{D}$ from Eq. (3.2.4) is calculated. The orift motion produced by electric, grad B, and curvature drifts is azlmuthal, so the partiole is simply rotated around the plasria axis between collisions. This azlmuthal drift does not affect alpha 
transport and can be neglected. We have chosen to neglect the curvature drift, since it is generally small compared to the other drift terms, but we include these other drifts to make the calculation more general. By neglecting the curvature drift, we can neglect the bounce motion, which allows us to use relatively large time steps between collision sampling events.

\subsection{Radial Guiding Center Drift}

The guiding center equations were derived under the assumptions that there is no interaction between the particle and the background plasma. This approximation is statistically valid for thermal particles in a uniform Maxwellian plasma where individual interactions do not affect the distribution function. But Coulomb collisions afiect the alpha orbit and they also affect the thermalization process. Collisions can be simulated as a discrete process in which drag, angle scatter, and dispersion events occur (see Chapter 4). The angle scatter and dispersion events are modeled as hard sphere interactions, whereas the drag is treated as a continuous event. The drag force acting on an alpha can be defined cy

$$
\frac{d \bar{v}}{d t}=v \bar{v}
$$

where $\nu=1 / \tau_{D}$. Although drag is continuous, it is not uniform because of the plasma temperature and density gradients. Large orbits, which are typina of energetlc alphas, enable the particle to sample plasma reglons which have different drag characteristles. Figure 3.1 shows 


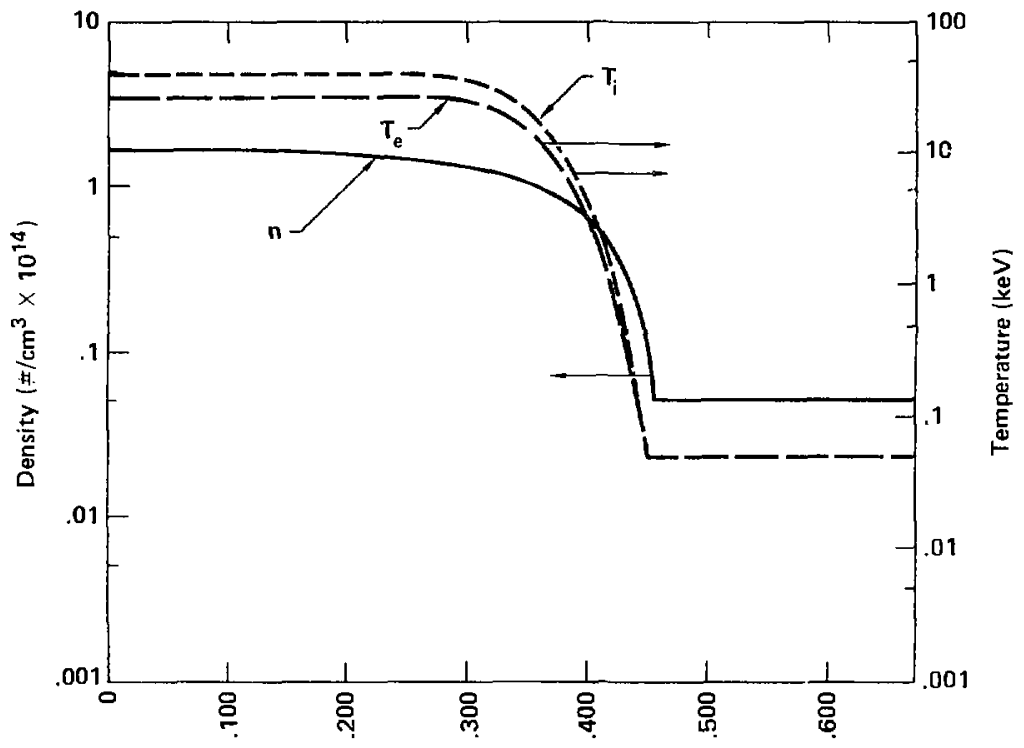

Plasma radius $(m)$

Figure 3.1 Assumed density and temperature profiles for a tandem mirror reactor with $r_{p}=0.46 \mathrm{~m}$, $n(0)=1.6 \times 10^{14} \mathrm{~cm}^{-3}, T_{i}(0)=40 \mathrm{keV}$, and $T_{\mathrm{e}}(0)=27 \mathrm{keV}$ 
assumed plasma density and temperature profiles for a tandem mirror reactor with a plasma radius of $0.46 \mathrm{~m}, \mathrm{n}=1.6 \times 10^{14} \mathrm{~cm}^{-3}$, $\mathrm{T}_{1}=40 \mathrm{keV}$, and $\mathrm{T}_{\mathrm{e}}=27 \mathrm{keV}$. Figure 3.2 shows the corresponding alpha drag time as a function of radius. The orag in the halo region, i.e. the region where the plasma density and temperature is significantly less than the corresponding values at $r=0$, is larger than the drag in the core because of the low halo temperature. The difference between the halo and core drag force that acts on an alpha orbit causes a radial guiding genter drift, and this drift can be derived using the formalism developed by Northrop. The radial drift occhrs in addition to the other guiding center drifts previously discussed.

The derivation of the radial drift starts with the equation of motion given by

$$
\frac{d \bar{v}}{d t}=\frac{q}{m}(\bar{v} \times \bar{B})-\frac{\bar{F}_{D}}{m}
$$

and neglects electrical, gravitational, and scattering collision forces. The drag rate across an orbit is not constant, so we expand $v$ in the small parameter $\delta$ and keep the flrst order terms

$$
v=v_{0}+\frac{\partial v}{\partial r} \rho+o(\delta)^{2}
$$

The equation of motion In terms of the radius vector $\bar{r}$ and $v$ is

$$
\ddot{\bar{r}}=\frac{q}{m} \dot{\bar{r}} \times \bar{\theta}-\left(v_{0}+\bar{\rho} \cdot \nabla \nu\right) \dot{\bar{r}}+o(\delta)^{2}
$$


56

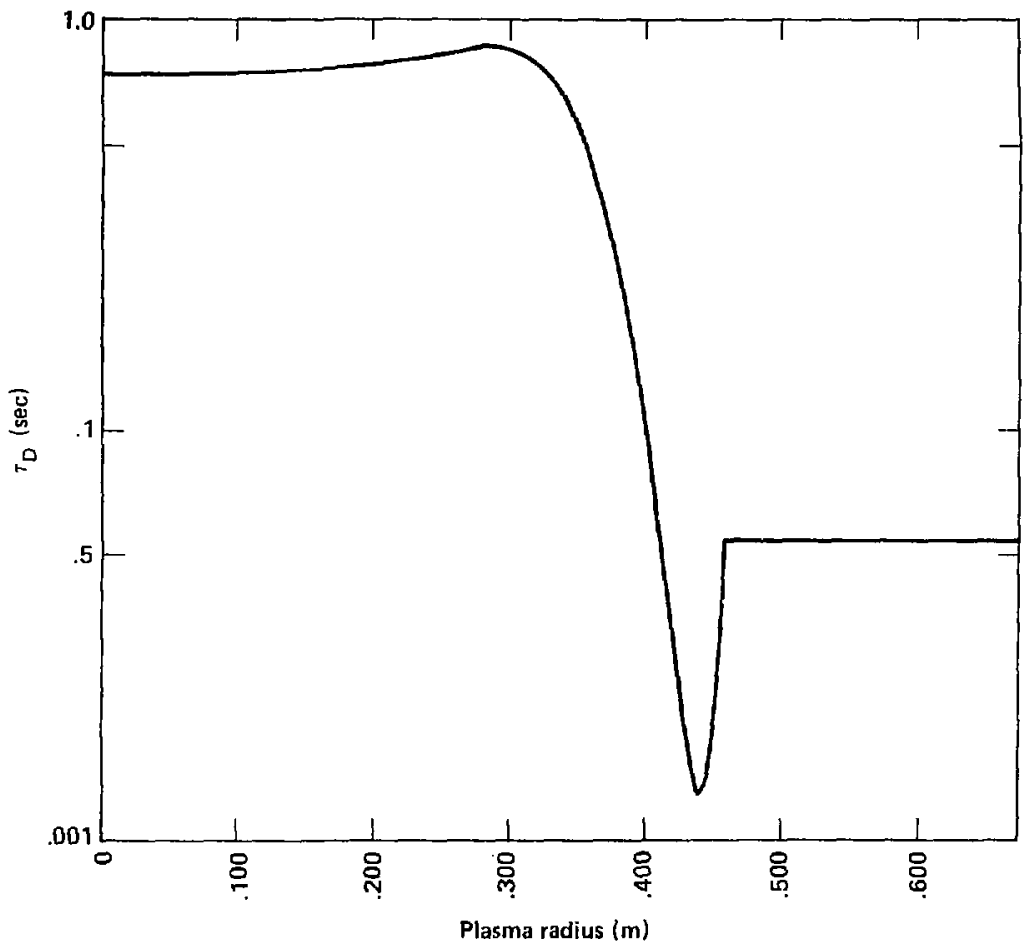

Figure 3.2 Drag profile for the density and temperature profile of Fig. 3.1 
The guiding center motion can be found by substituting $\bar{R}+\bar{\rho}$ for $\bar{r}$ and is

$\ddot{\vec{R}}+\ddot{\bar{\rho}}=\frac{q}{m}[(\dot{\bar{R}}+\dot{\bar{\rho}}) \times \vec{B}]-v_{0}(\dot{\bar{R}}+\dot{\bar{\rho}})-\rho \frac{\partial \nu}{\partial r} \dot{\vec{R}} \sin \phi-\beta \frac{\partial v}{\partial r} \dot{\bar{\rho}} \sin \phi+o(\delta)^{2}$

where $\bar{p}=\rho,\left(\hat{e}_{2} \sin \phi+\hat{e}_{3} \cos \phi\right)$. The terms involving $\sin \phi$ arise because the gradient of $v$ is in the radial direction parallel to the assumed $\hat{e}_{2}$ direction and therefore $\rho \cdot \nabla v$ results in terms containing only $\sin \phi$. The guiding center motion is derived by averaging Eq. (3.4.5) over a gyroperior. Terms cortaining $\sin \phi$ and $\cos \phi$ average to zero. Niner terms which are not functions of premain unchanged in the average. The guiding center equation now becomes

$$
\ddot{\bar{R}}=\frac{q}{m} \dot{\bar{R}} \times \bar{B}-\frac{p^{2} w_{c}}{2 \pi} \int_{0}^{2 \pi} \frac{d v}{d r} \sin ^{2} \phi d \phi \hat{e}_{3}
$$

In order to discuss the parallel and perpendicular motion caused by the irag gradierts, let the integral in Eq. $(3.4 .6)$ be equal to $\left\langle\dot{\bar{V}}_{D}\right\rangle$. The parallel motion is fornd by taking the scalar product of $\ddot{\bar{R}}$ with $\hat{e}_{1}$, i.e.

$$
\ddot{\bar{R}} \cdot \hat{e}_{1}=\frac{q}{m}(\dot{\bar{R}} \times \bar{B}) \cdot \hat{e}_{1}-\left\langle\dot{\bar{V}}_{D}\right\rangle \hat{e}_{3} \cdot \hat{e}_{1}=0 \cdot(3.4 .7)
$$


Therefore, a radial drag force doss not aiter the parallel motion of the alpha guiding center. The drift motion is found from the vector product of $\ddot{\bar{R}}$ with $\hat{e}_{i}$, i.e.

$$
\ddot{\bar{R}} \times \hat{e}_{1}=\frac{q}{m}(\dot{\bar{R}} \times \overline{\mathrm{B}}) \times \hat{e}_{1}-\left\langle\dot{\bar{V}}_{D}\right\rangle_{e_{3}} \times \hat{e}_{1} .
$$

The drift velocity then becomes

$$
\dot{\vec{R}}_{D}=\dot{\vec{R}}-\left(\dot{\bar{R}} \cdot \hat{e}_{1}\right) \hat{e}_{1}=-\frac{1}{\omega_{c}} \ddot{\bar{R}} \times \hat{e}_{1}-\frac{\dot{V}_{D}}{\omega_{c}} \hat{e}_{2}+O(\delta)^{2} \text {. }
$$

The first term on the right hand side of Ea. (3.4.9) is the radial acceleratic. of the guiding center. This acceleration, to zero order, can be produced by a radial electric field and has been considered in the guiding centar theory. There are no acceleration terms of order $\delta$ or lower introduced by drag, so that $\left(\frac{1}{w_{c}}\right) \ddot{\bar{R}} \times \hat{e}_{i}$ is $0(5)^{\hat{c}}$ or higher. The guiding center drift velocity is then reduced to

$$
\dot{\bar{R}}_{\mathrm{D}}=-\frac{\left\langle\dot{\bar{V}}_{\mathrm{D}}\right\rangle_{\mathrm{C}}}{\hat{\mathrm{e}}_{2}}
$$

The radial direction of the drift is dependent on $\frac{d v}{d r}$ which depends on the density and temperature profiles. 


\subsection{Radial Drift Simulation}

The radial guiding center drift occurs when there are gradients In drag over a Larmor orblt, as seen from Eq. (3.4.6). Besides this radial drift, the Larmor radius decreases due to the energy loss associated with the drag process. A sketch of the alpha orbits in the uniform core plasma and in the halo region is shown in Fig. 3.3. In simulating the alpha particle motion, the guiding center is moved radially between collision sampling events and the orblt shrinks at the collision event. The radial movement depends in evaluating the integral in Eq. (3.4.6), which is not well behaved since $\frac{d v}{d r}$ can have a large oscillation and the integrand is infinite at the end points. The end point singularity occurs whenever $\frac{d v}{d r}$ is functionally dependent on $r$. The deceleration $\ddot{\bar{B}}$ is continuous and non-zero over the orbit; therefore, even though there are no radial forces acting on the particle ven $\phi$ is $0, \pi, 2 \pi, .$. , there is a finite limit to $\frac{d v}{d r}$ $\sin ^{2} \phi$ as $\phi$ approaches these critical values. A plot of $\frac{d v}{d r}$ for the profiles in Figs. 3.1 and 3.2 is shown in Fig. 3.4. The particle position $\bar{r}$ is defined in terms of $\bar{R}$ and $\bar{\rho}$ in Fig. 3.5 and the magnitude of $\bar{r}$ is

$$
r=\left(p^{2}+\rho^{2}+2 R \rho \sin \phi\right)^{1 / 2} \text {. }
$$

The integral can now be transformed into 


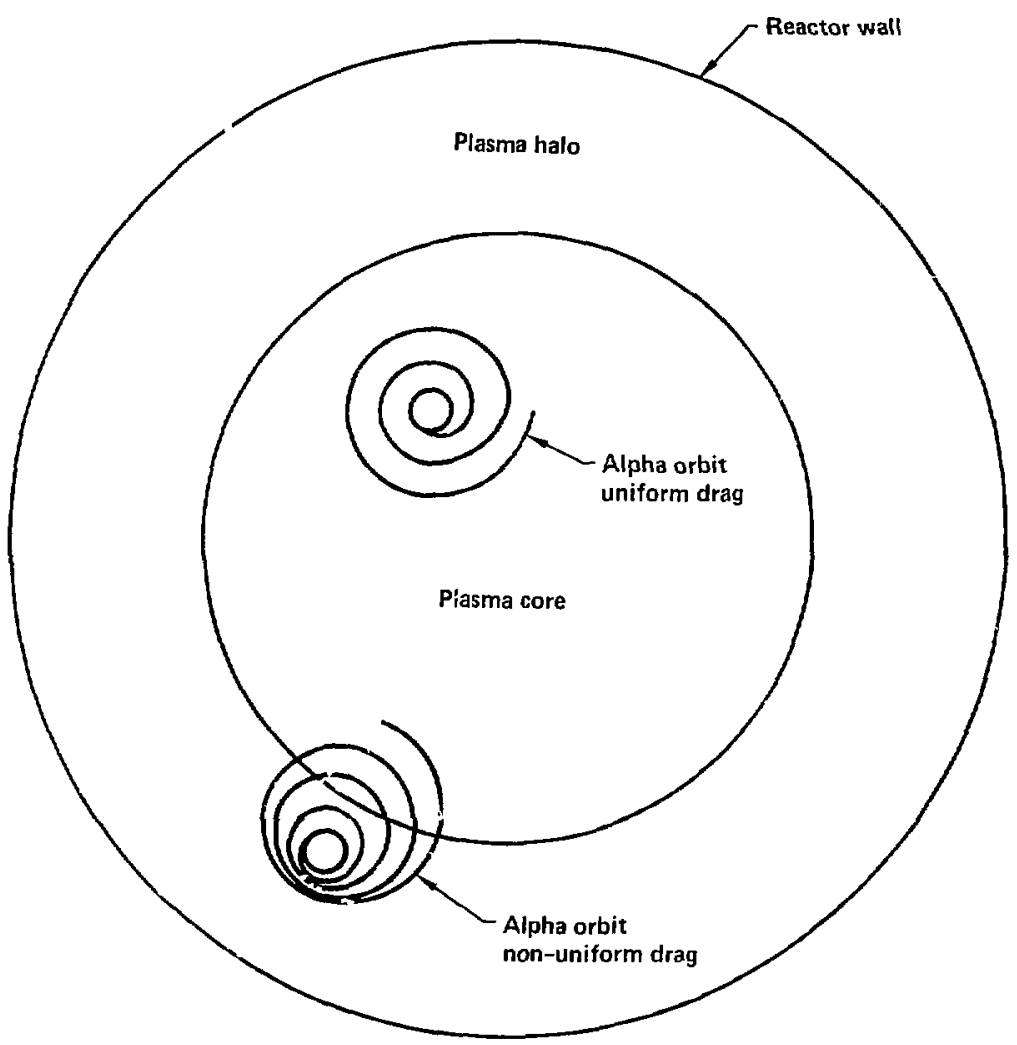

Figure 3.3 Alpha particle orbits in the uniform core plasma and in the drag gradient region between the core and halo 


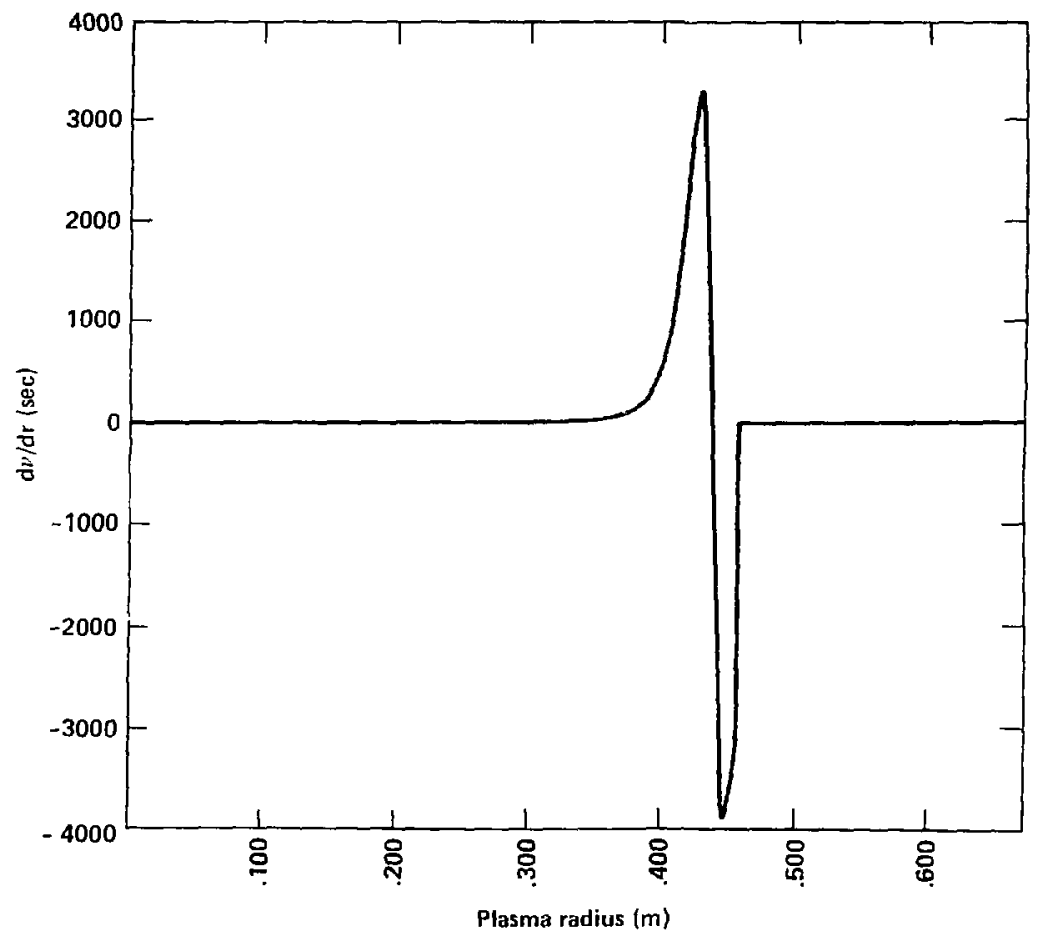

Figure 3.4 Drag gradient profile for the density and temperature profile of Fig. 3.1 


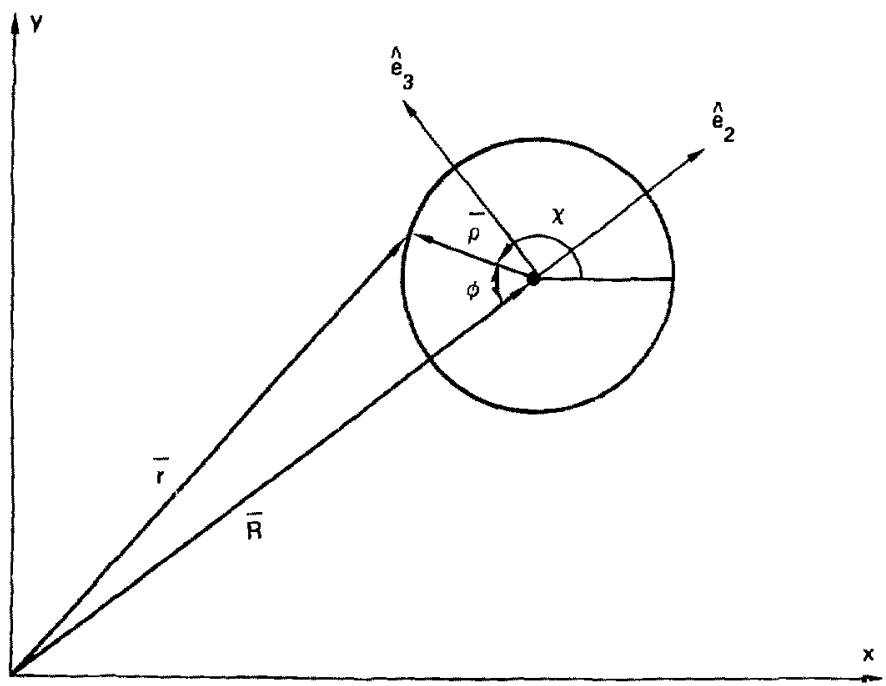

Figure 3.5 Relation between the particle position ector $(\bar{r})$ and the gulding center $(\overline{\mathrm{R}})$ and Larmor radius $(\overline{\mathrm{p}})$ vectors 
$\left\langle\dot{\bar{V}}_{D}\right\rangle=\frac{1}{2 \pi} \frac{\rho}{R} \int_{R-\rho}^{R+\rho} \frac{d v}{d r} \frac{\left[r^{2}-\left(R^{2}+p^{2}\right)\right]^{2}}{4 R^{2} p^{2}} \frac{r d r}{\left(1-\frac{\left[r^{2}-\left(R^{2}+p^{2}\right)\right]^{2}}{4 R^{2} \rho^{2}}\right)^{1 / 2}}$

Because the integrand is infinite at the I points, a Gauss-Cheryshrov numerical solution of $\mathrm{Eq} .(3.4 .12)$ can be obtained when the linits of the integral are changed to \pm 1 . Let the variable $t$ equal $\frac{r^{2}-\left(R^{2}+p^{2}\right)}{2 R \rho}$ and let $\frac{d v}{d t}=\frac{d v}{d r} \frac{d r}{d t}$. The guiding center drift becomes

$$
\begin{aligned}
\dot{\bar{R}}_{D} & =\frac{\left\langle\dot{\bar{V}}_{D}\right\rangle}{\omega} \\
& =\frac{\rho}{\pi} \int_{-1}^{1} \frac{d v}{d t} \frac{\left(R^{2}+\rho^{2}+2 R \rho t\right)^{1 / 2}}{i t} \frac{t^{2} d t}{\left(1-t^{2}\right)^{1 / 2}} .
\end{aligned}
$$

An exact evaluation of $\overline{\bar{H}}_{D}$ would raquire exact knowledge of $\frac{d v}{d t}$; but since a numerical scheme is used, the evaluation of $\frac{d v}{d t}$ is required at specified values of $t$. Therefore, $\frac{d v}{d r}$ can be evaluated for values of $r$ equal $\left(R^{2}+p^{2}+2 R \rho t\right)^{1 / 2}$. Using these relations, the above integrand can be evaluated if the function $\frac{d v}{d r}$ is well behaved.

The end point singularity in the integrand is compounded by a large swing in $\frac{d v}{d r}$ near the plasma boundary. This oscillation is caused by the assumed density and temperature proflles, whi wh are discussed in Chapter 5. If this swing occurs over a short distance cimpared with the alpha orbit diameter, then the integral given by Eq. (3.4.13) 
requires an excessive number of quadrature points. An example of the temperature and density profiles that produce large swings in $\frac{d v}{d r}$ is shown in Fig. 5.6. Large peaked swings indicate a sharp plasma halo boundary which may be physically impossible to maintaln. Therefore, by choosing reasonable radial density and temperature profiles, the alpha radial drag drift can be calculated using a relatively small number of points.

The solution to Eq. (3.4.13) depends on determining the number of quadrature points to be used in the Gauss-Chevyshev numerical integration. A number of experiments were performed using the radial temperature and density profiles shown in Fig. 3.2 to determine the number of polnts needed to accurately calculate the integral. It was found that in the plasma core region ( $r$ less than $0.7 r_{p}$ ) four quadrature points are needed to obtain a radial drift within one percent of the determined asymptotic value. At larger radii where $\frac{d v}{d r}$ is fluctuating, the number of quadrature points increased to 16 to obtain the same accuracy. 


\section{PART 4 \\ MONTE CARLO TECHNIQUE}

\subsection{Introduction}

The Monte-Carlo technique is applied to alpha particle Initialization and the alpha thermalization process with the plasma. Alpha particles are born in the plasma over a narrow energy band, and we assume that all alphas are born with the mean energy of $3.52 \mathrm{MeV}$. The initial alpha phase space coordinates are determined by Monte-Carlo techniques by sampling from appropriate distribution functions. This sampling technique is discussed in Sections 4.2 and 4.3 . As the alpha particle slows down, it diffuses in a random manner through pl: ise space. This random walk process, which changes the particles velocity and position coordinates, is modeled by the Boltzman equation using the Fokker-Planck collision operator. The collision operator contalns a continuous slowing down or drag term plus scattering terms. These terms are an accumulation of many interactions over a relatively long time period, but they can be simulated as one event occurring over an Infinitesimally short time. The detalls of modeling the collisions in a Monte-Carlo scheme are presented in s-ation 4.4 .

\subsection{Alpha Initialization}

Alpha particleg born in the plasma have their phase space positions which depend on the background plasma denslty, temperature, and the detalis of the D-T collision. The details of the fusion reactions are not needed to simulate the alpha birth position in phase 
space. The alpha birth coordinates are selected from appropriate probability distribution functions. For example, since we know the speed of the alpha, we only need to specify the pltch angle $\psi$ and an azlmuthal or phase angle $\phi$ to deternine the velocity space coordinates. There is no preferential birth direction, so the probability distribution for both pitch and phase angles is uniform. The pitch angle is chosen from 0 to $\pi$, and the phase angle is chosen from 0 to $2 \pi$. These velocity components lie on a velocity sphere of radius equal to $|\vec{v}|$, and the probablilty (P) that a parificle has a velocity component between 0 and $\psi$ and between 0 and $\phi$ is

$$
\mathrm{P}=\frac{\int_{0}^{\phi} \int_{0}^{\psi} v^{2} \sin \psi c \psi \mathrm{d} \phi}{\int_{0}^{2 \pi} \int_{0}^{\pi} v^{2} \sin \psi d \psi d \phi}
$$

Since the pitch and phase angles are independent of each other, we define the pitch probability $\left(\mathrm{P}_{\psi}\right)$ and the phase probability $\left(\mathrm{P}_{\phi}\right)$ as

$$
P_{\psi}=\frac{1}{2}(1-\cos \psi)
$$

and

$$
P_{\phi}=\frac{\phi}{2 \pi}
$$

Since neither $\psi$ nor $\phi$ are known, the value of $P_{\psi}$ and $P_{\phi}$ can be chosen from a uniform random distribution between 0 and 1 , and the values of $\psi$ and $\phi$ can be found from 


$$
\begin{aligned}
& \psi=\cos ^{-1}\left(1-2 P_{\psi}\right) \\
& \phi=2 \pi P_{\phi} .
\end{aligned}
$$

Therefore, by choosing two random numbers, the values of $\psi$ and $\phi$ can be determined.

Particle position in a reactor is determined by a similar process. A cylindrical coordinate system is used, in which z lies along the axis of the machine. The probability of the alpha being born at any location is given bj

$$
p=\frac{\int_{V} \dot{\mathrm{N}}_{a} d V}{\int_{V} d V}
$$

where the integral is performed over the volume and $\dot{N}_{\alpha}$ is the D-T reaction rate. The probability that the alpha is born in $\mathrm{dV}$ can be defined as the product of the probabilities of the alpha being born at any one of the three coordinate positions, 1.e.,

$$
P=P_{r} P_{z} P_{x} \text {, }
$$

where $P_{r}, P_{z}, P_{X}$ are the probabilities that the particle will be born between $r$ and $r+d r, z$ and $z+d z$, and $x$ and $x+d x$. The birth probabllity at any azimuthal position ( $x$ ) is simflar to Eq. (4.2.5) and is given by 


$$
X=2 \pi P_{X} .
$$

The alpha birth position along $z$ can be set to one since the particle bounce time is signiflcantly less than the collision saduj: $\mathrm{ng}_{\mathrm{B}} \mathrm{time}$, and the particle birth position along $\mathrm{z}$ is simulated at the plisma midplane.

The radial birth position is more complex because it is dependent on the $\mathrm{D}-\mathrm{T}$ reaction rate. The reaction rate, given by

$$
\dot{N}_{\alpha}=n_{D} n_{r}\langle\sigma v\rangle
$$

is a cunction of radius through the density and microscopic cross section 〈ov〉. The cross section is temperature dependent, w1th the temperature being a function of radius. The probability of finding a particle between 0 and $r$ is then

$$
P_{r}=\frac{\int_{0}^{r_{n} n_{D} n_{T}\langle\sigma v\rangle r d r}}{\int_{0}^{r_{p}} n_{D} n_{T}\langle\sigma v\rangle r d r} .
$$

By randomly choosing $P_{r}$ from a uniform distribution between 0 and 1 , we can solve for $r$ analytically after performing the integraclons. Solving for $r$ in thls manner is not trivlal, since the plasma density and temperature, and therefore the reaction rate, are complicated cunctions. We solve this problem by numericaliy integrating 


$$
\int_{r_{1}}^{r_{1}} 1+2 n_{D} n_{T}\langle\sigma v\rangle r d r
$$

uging simpson's rule, where $1=0,2,4,6, \ldots . N$, and $N$ is even and is equal to

$$
\frac{r_{N}}{r_{i+1}-r_{i}} \text {. }
$$

Using this technique, we develop a table in which values of the integral, given by

$$
s_{j+1}=s_{j}+\int_{r_{1}}^{r_{1+2} n_{D} n_{T}\langle\sigma v\rangle r d r},
$$

where $j$ is $i / 2$, are kept. The total integral,

$$
s_{j N}=\int_{0}^{r} p n_{D} n_{T}\langle\sigma v\rangle r d r
$$

where $j N=\frac{N}{2}$, is therefore the last value in this table. Values of $\mathrm{P}_{\mathrm{r}} \mathrm{S}_{\mathrm{jN}}$ can be compared with the table values, and $r$ can be found by linear interpolation. This technlque $1 \mathrm{~s}$ simple and accurate and only requires the numerical evaluation of the lntegral along with an interpolation scheme, whereas other root flnding schemes require function evaluations, 1terations, and the derivative of the function. 
A typical radial alpha birth profile found from this tecr.nique is shown in Fig. 4.1 for the base case reactor parameters given in Table 1.1. Thls Monte-Carlo birth distribution was made using 7000 alpha particles and is compared to a theoretical birth distribution determined from Eq. (4.2.10), where $P_{r}$ is calculated from $P_{r}=S_{j} / S_{j N}$. The results of numerical experiments show that simulations using 1000 particles produce energy deposition profiles that are smooth, but the particle birth distribution, as shown in Fig. 4.2, has more scatter than simulations using more alphas. This experiment also produced accurate thermal alpha particle distribution.3. Therefore, we chose to use 1000 alpha particles in the remaining alpha transport studies. The scatter that is seen In Eigs, 4.1 and 4.2 is a function of the radial bin size compared to the number of alpha particles started, and it is also dependent on the seed value $B 1$ ven to the random number generator. Both Figs. 4.1 and 4.2 show that the fraction of alpha particles born on axis is zero. This valie must be zero, since the probability density function is volume weighted and the reacting volume approaches zero as the radius goes to zero. The birth profile also goes to zero as the radius inereases because the temperature and density of the plasma lons decrease, which causes the reaction rate to approach zero. In order to check the birth distribution profile in velocity space, we can compare the expected number of particles born in the loss cone to that produced by the Monte-Carlo technique. By reducing the potential barrier to zero and malntaining high mirror ratlos, we can show that the expected loss rate for a mirror ratio of 8 is 


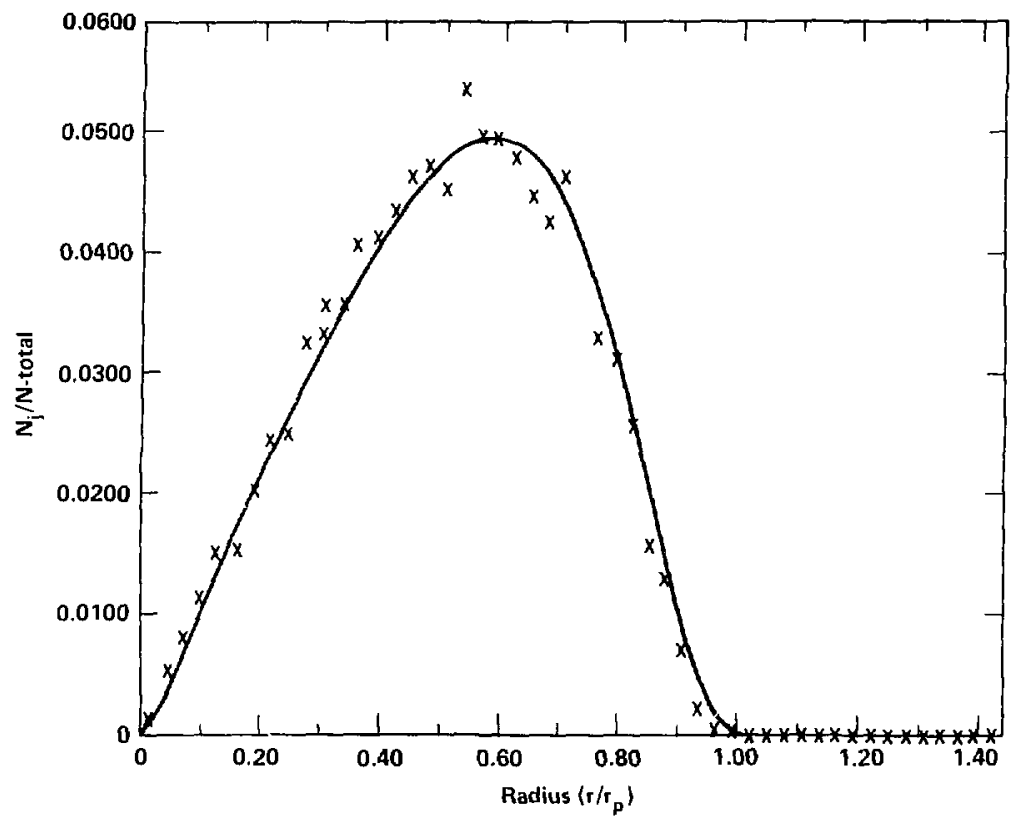

Figure 4.1 Alpha particle radial birth profile determined by 'Tonte-Carlo techniques for 7000 alpha particles $(x)$ and zompared with a theoretical birth distribution (solid line) 


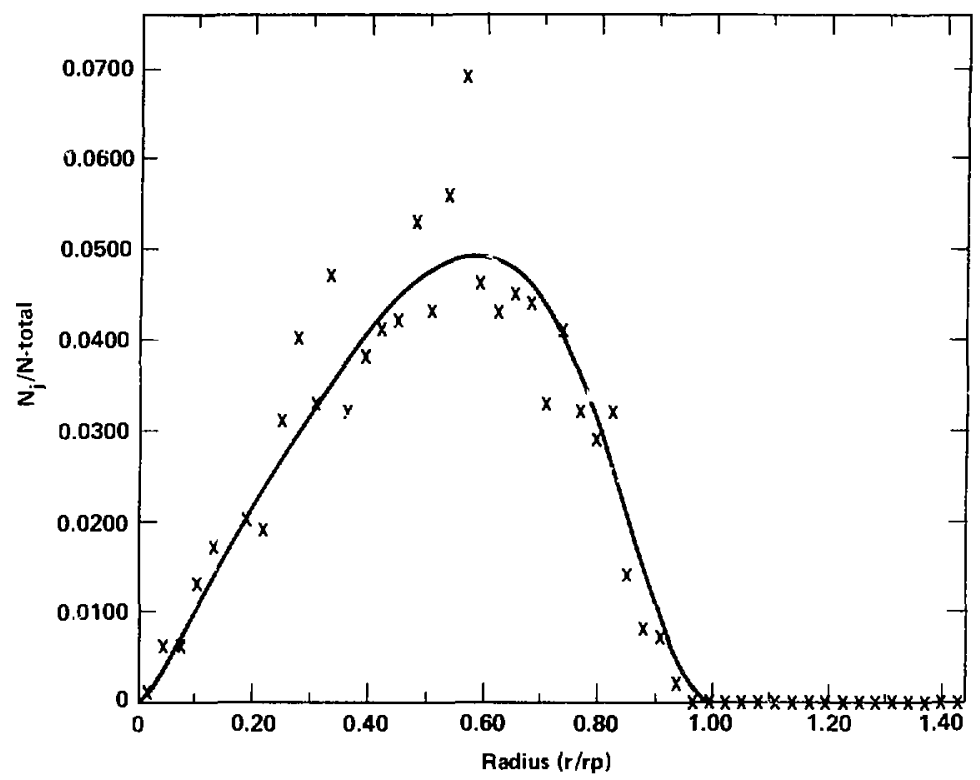

Flgure 4.2 Alpha particle radial birth profile determined by Monte-Carlo techniques for 1000 alpha particles $(x)$ and compared with a theoretical birth distribution (solid line) 
approximately equal to $1 / 2 \mathrm{R}$ or $6.25 \%$; this compares with the loss rate of $5.93 \%$ produced by the code using 1000 alpha particles.

\subsection{Alpha Particle Guiding Center and Drift}

Once the particle position is specified, its guiding center position and drift velocities must be determined. A sketch of the alpha motion along with the pertinent coordinate systems is shown in Fig. 4.3. The particle osition $(\bar{r})$ in the laboratory coordinate system is known along with the pitch and phase angle. The pitch angle in the laboratory system and in the gufding center (GC) system are equal at the plasma midplane since the flux lines are parailel to the z-axis. The velocity in the GC system is given by

$$
\vec{v}=v \sin \psi \cos \phi \hat{\mathbf{i}}+v \sin \psi \sin \phi \hat{\mathbf{j}}+v \cos \psi \hat{\mathrm{k}} \cdot(4.3 .1)
$$

This velocity is equal to the velocity in the laboratory system at the plasma midplane because the GC and laboratory systems are parallel. The velocity parallel to the magnetic field is found by taking the scalar product of $\bar{v}$ and $\bar{B} / \bar{B}$ as

$$
\bar{v}_{\|}=\left(\bar{v} \cdot \frac{\bar{B}}{B}\right) \frac{\bar{B}}{B}=\left(\bar{v} \cdot \hat{e}_{1}\right) \hat{e}_{1} \cdot
$$

The perpendicular velocity is then given by

$$
\bar{v}_{\perp}=\bar{v}-\bar{v}_{\|}=\bar{v}-\left(\bar{v} \cdot \hat{e}_{1}\right) \hat{e}_{1},
$$




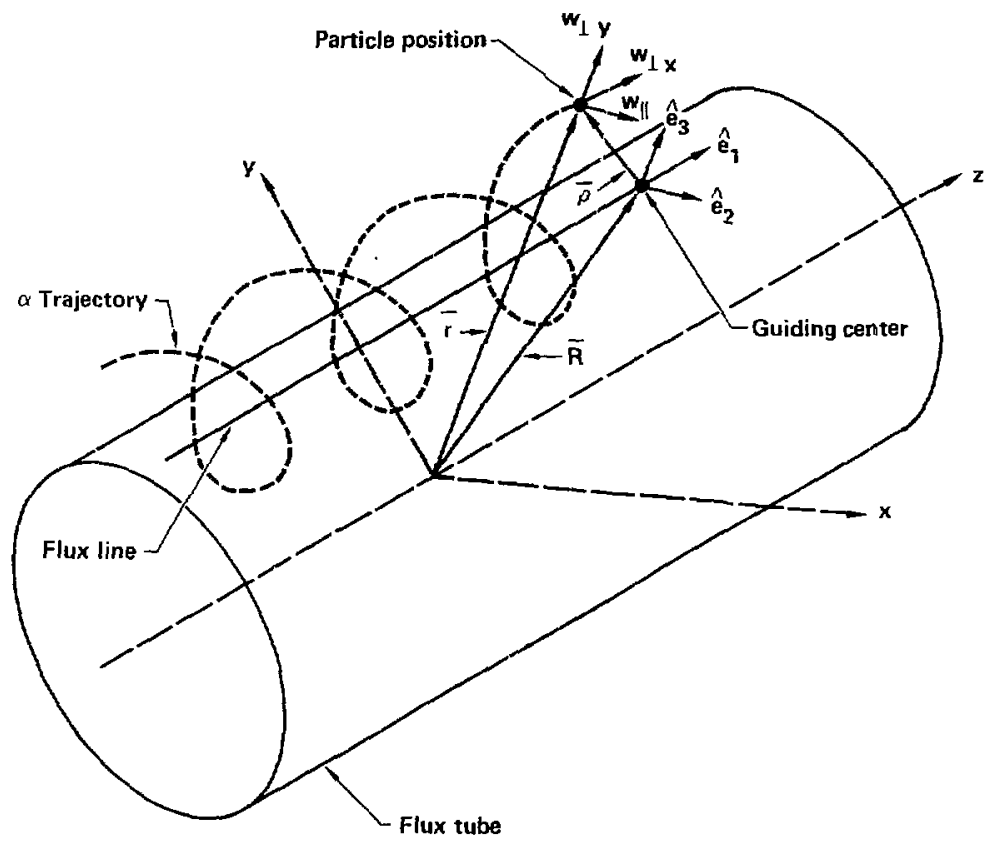

Figure 4.s Coordinate systems in the center cell of a tandem mirror reactor, Laboratory system $(x, y, z)$, Guiding Center system $\left(e_{1}, e_{2}, e_{3}\right)$, and Particle system $\left(w_{\|}, w_{\perp x}, w_{1 y}\right)$ 
and $\bar{v}_{1}$ can be used to find magnetic moment $\mu$, and the Larmor radius $p$. Equations (4.3.2) and (4.3.3) can be used to find the orfft velocity without drag effects, and these equations can be integrated to find the guiding center position,

$$
\overline{\mathrm{R}}=\overline{\mathrm{r}}-\bar{\rho} \text {. }
$$

The initial calculation of $\bar{R}$ is sensitive to the magnetic field changes over the large alpha orbit. This effect occurs because the initial value of $\rho$ is calculated using $\beta$ at $\bar{r}$, whereas the value of $\beta$ at $\bar{R}$ is needed to determine $p$. The velocities are not affected by $B$ because, as argued in Chapter 2, the plasma pressure has little effect on field line curvature and, therefore, vacuum values of $\bar{B} / \mathrm{B}$ accurately define the direction of the unit vector parallel to $\bar{B}$. Since $B$ can change over a Larmor orbit, a Newton iteration scheme 1s used to initially locate $\bar{R}$. This iteration is performed by defining the function fin as

$$
f n=|\bar{R}|-\left|\bar{r}-\frac{\vec{\rho}}{\left[1-B_{o} f\left(\frac{r}{r_{p D}}\right) f\left(\frac{r}{r_{p T}}\right)\right]^{1 / 2}}\right|,
$$

where $\bar{D}$ is defined in terms of $\bar{v}_{1}$ and $B_{v}(R)$. The iteration is performed on $|\bar{R}|-\left|\bar{r}-\bar{\rho}_{a}\right|$ because the magnitude of the orbit determines the effect of $B$ on the guiding center position. The iteration scheme is defined as 


$$
R_{n+1}=R_{n}-\frac{f n}{\frac{d f n}{d r}}
$$

This method converges within ten 1terations when an error criterion of $1 \times 10^{-4}$ is used.

\subsection{Particle Collisions}

\section{4 .1 Velocity Transformation}

After the guiding center position, parallel velocity, and drift velocity are known, the particle information is extraneous and is not kept except for statistical computations. The guiding center is tracked up to a collision sampling time, and the particle position is reconstructed using Monte-Carlo techniques. In this situation, $\bar{R}$ is known and we need to find $\bar{r}$. The particle phase angle, $\phi$, is chosen from a uniform distribution given by Eq. (4.2.5) to statistically compensate for sampling the collisions at the plasma midplane. The magnetic moment, $\mu$ is used to find $|\bar{v}|$ from Eq. $(1.1 .2)$ and the magnitude of the Larmor radius is found from Eq. (1.3.6). The particle position in laboratory coordinates can be found from $\bar{r}=\bar{k}+\rho_{0} \cos \phi \hat{e}_{2}+\rho_{0} \sin \phi \bar{e}_{3}$.

The velocity is transformed to the particle coordinate $(w)$ system in which the velocity before the collision sampling, defined as WH $(F i g, 4.4)$, is given by

$$
w_{\|}=\bar{v} \text {. }
$$




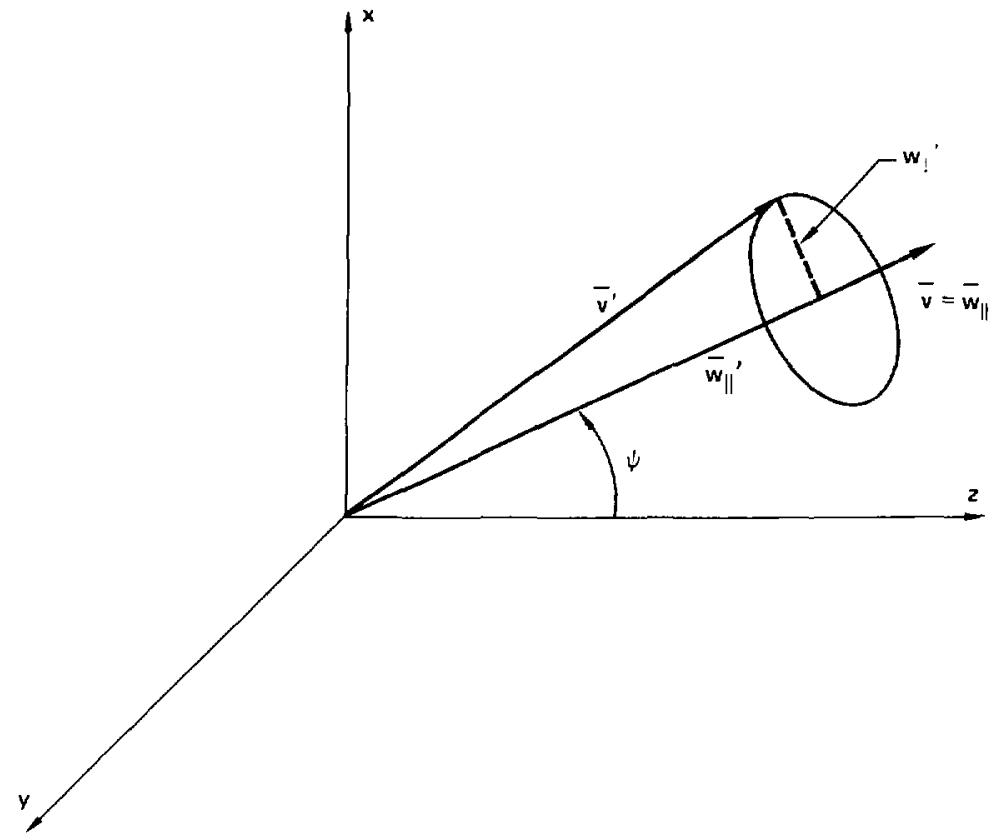

Figure 4.4 Relation between the Laboratory and Particle Coordinate system before and after (primed) a collision 
After the collision, the alpha has a parallel, $w_{\|}$, component and a component perpendicular to $w_{\|}$or $w_{1}$. The perpendicular direction is defined in such a way that the transformation from the $w$ to the laboratory system is computationally quick and accurate. Since we are at the plasma midplane, the laboratory and the guiding center systems differ only by the displacement $\bar{R}$. The perpendicular velocity $\left(v_{1}\right)$ to $\hat{e}_{1}$ has an angular direction of $90+\phi$. The parallel velocity before the collision in the laboratory coordinate system is

$$
\begin{aligned}
& v_{\| x}=w_{\|} \sin \psi \cos \phi, \\
& v_{\| y}=w_{\|} \sin \psi \sin \phi, \\
& v_{\| z}=w_{\|} \cos \psi .
\end{aligned}
$$

After the collision is sampled, the parallel velocity is changed to $w_{i}^{\prime}$ and the perpendicular components are $w_{i x}$ and $w_{i y}^{\prime}$. The angle from $w_{1}$ to the laboratory $z$-axis is $90+\psi$ where $\psi$ is the initial pitch angle. This gives the transformation of $w_{1 x}$ into the laboratory coordinate system as

$$
\begin{aligned}
& v_{I x x}=w_{I x}^{\prime} \sin (90+\psi) \cos \phi=w_{I x}^{\prime} \cos \psi \cos \phi, \\
& v_{I x y}=w_{I x}^{\prime} \sin (90+\phi) \sin \phi=w_{I x}^{\prime} \cos \psi \sin \phi,(1.4 .1 .3) \\
& v_{I x z}=w_{I x}^{\prime} \cos (90+\phi) \quad=w_{I x}^{\prime} \sin \psi .
\end{aligned}
$$


The direction of $w_{\perp y}^{\prime}$ is the defined by $w_{\perp y}^{\prime}=w_{\|}^{\prime} \times w_{\perp x}^{\prime}$ and the transformation to laboratory coordinates given by

$$
\begin{aligned}
& v_{\perp y x}=w_{\perp y} \sin \phi, \\
& v_{\perp y y}=w_{\perp y} \cos \phi, \\
& v_{\perp y z}=0 .
\end{aligned}
$$

This transformation in matrix form is then

$$
\left|\begin{array}{c}
v_{x} \\
v_{y} \\
v_{z}
\end{array}\right|=\left|\begin{array}{rrr}
v_{\perp x x}+v_{\perp y x}+v_{\| x} \\
v_{\perp x y}+v_{\perp y y}+v_{\| y} \\
v_{1 x z}+v_{\perp y z}+v_{\| z}
\end{array}\right|=\left|\begin{array}{rrr}
\cos \phi \cos \phi & -\sin \phi \sin \psi & \cos \phi \\
\cos \phi \sin \phi & -\cos \phi \sin \phi & \sin \phi \\
-\sin \phi & 0 & \cos \phi
\end{array}\right|\left|\begin{array}{c}
w_{1 x}^{1} \\
w_{1 y}^{\prime} \\
w_{\|}^{\prime}
\end{array}\right|
$$

This transformation allows collisions to be sampled in the particle coordinate system and the resulting velocities transformed into laboratory coordinates.

\subsubsection{Collision Sampling Time}

Collisions are sampled at intervals determined by the alpha particle drag, scatter and energy exchange times. These characteristic t.imes vary with alpha speed and plasma density and temperature, for example, $\tau_{D}$ in the core is more than ten times greater than $\tau_{D}$ in the halo. Since energetj.c alpha orbits can extend over large plasma radial cistances, the collision sampling time ( $\Delta t$.$) depends on characteristic$ 
times around the alpha orbit. The value of $\Delta t$ is computed using the smallest value of $T_{D}, T_{E}$, and $\tau_{\perp}$, calculated at the particle position when

$$
|\overline{\mathrm{R}}|+|\vec{\rho}| \leq \mathrm{r}_{\mathrm{p}}
$$

When Eq. (4.4.2.1) is not satisfied, the sampling time is computed from the smallest value of $\tau_{D}, \tau_{E}$ and $\tau_{\perp}$ calculated at $\bar{r}, \tau_{D}$ calculated in the halo, and $\tau_{E}$ and $\tau_{I}$ calculated at $\bar{r}=0$. The smallest

characteristic sampling time is then multiplied by .025 , which has been determined by numerical experiments to be an appropriate scaling factor, to determine $\Delta t$. This sampling time is then modified to colncide with the time the particle is at the plasma midplane by obtaining the integer value of $.025 v_{\|} \Delta t / L$ and then multiplying this integer by the bounce time $\left(L / v_{\|}\right)$.

\subsubsection{Mass ConsLderations}

The plasma is composed of electrons, fuel ions, and alpha particles. We assume that an average ion atomic mass of 2.5 a.m.u. can be used to represent the fuel luns, since the deuterium and tritium are similar in charge, number density, and temperature. This assumption Implies that the speed difference between the $D-T$ ions is negligible in determining alpha-ion interactions. This assumptioit becomes exact for the drag collision process when the D-T densities are equal and when their speeds are equal. By averaging the D-T mass, the reacting plasma becomes a two-component plasma made of electrons and ions; this reduces 
the computational time needed to follow alphas from birth to thermali zation.

The alpha mass is not included in the average lon specie that makes up the plasma. We assume that the alpha is a separate fluid that interacts only with the background ion and electron plasma. This assumption is justified becaise typical alpha particle concentrations are expected to be approximately .05 in present tandem mirror reactor studies. ${ }^{34}$ since the alpha concentration is kept low in these studies, we have assumed that a low alpha concentration will be maintained in the generic reactor deslgn. Therefore, the average plasma ion mass neglects the contribution due to the thermalized alpha particles.

Consistent with the assumption that the alpha concentration is low, alpha-alpha interactions are neglected. The alpha particle initially drags down on plasma electrons until it's energy is less than $15 \mathrm{~T}_{\mathrm{e}^{\cdot}} 7,43$ After this, the number of alpha-ion $(\alpha-i)$ and alpha-alpha $(\alpha-\alpha)$ interactions increases. The ratio of $\alpha-\alpha$ to $\alpha-i 90^{\circ}$ scattering times is approximated by

$$
\frac{\tau_{\alpha \alpha}}{\tau_{\alpha i}}=\frac{n_{i}}{z_{\alpha}{ }^{2} n_{\alpha}}
$$

Low alpha concentrations imply that the $\alpha-\alpha$ scattering times are much longer than the $\alpha-i$ scattering time. When $\tau_{\alpha \alpha}$ is long compared to $\tau_{\alpha 1}$ and since alpha-alpha Interactions have little effect on the equilibrium alpha distribution functions, we neglect the alpha-alpha collisions. 


\section{4 .4 Drag Collision Sinulation}

Alpha particles slow down by being dragged down in energy by the surrounding plasma and at the same time they suffer large angle scattering and parallel dispersion events. Spitzer ${ }^{32}$ has derlved the average drag rate $\left\langle\Delta w_{\|}\right\rangle$acting on a particle which is

$$
\left\langle\Delta w_{\|}\right\rangle=-\sum_{f=1, e} A^{A_{f}{ }^{2}}\left(1+\frac{m}{m_{f}}\right) G\left(l_{f} w\right)
$$

where the summation $1 \mathrm{~s}$ over the background plasma ions and electrons. The subscript $f$ refers to the field or plasma particles and \& $f$ is the reciprocal of the ion or electron velocity defined by

$$
\ell_{f}=\left(\frac{2 T_{f}}{m_{f}}\right)^{-1 / 2}
$$

In uniform plasmas, $A_{D}$, Biven by

$$
A_{D}=\frac{8 \pi q^{4} n_{f^{2} f^{2} z^{2} L n \Lambda_{f}}}{\epsilon_{o m^{2}}^{2}},
$$

is a constant relating the charges, density, and coulomb logarithm of the plasma and alpha test particle. The argument of the $G$ is a dimensionless ratio of alpha to field particle speed, and $G$ is 


$$
G(x)=\frac{\Phi(x)-x \Phi^{\prime}(x)}{2 x^{2}}
$$

where

$$
(4.4 .4 .4)
$$

$$
\Phi(x)=\frac{2}{\sqrt{\pi}} \int_{0}^{x} e^{-y^{2}} d y
$$

In the limit as $x$ goes to zero, 1.e. as the plazma partlole speed is much greater than the alpha particle speed, the value of $G$ can be approximated by

$$
G(x)_{x \rightarrow 0}=\frac{2}{3} \frac{x}{7 \pi}
$$

By substituting Eq. $(4.4 .4 .5)$ into Eq. $(4.4 .4 .1)$ and simplifying, it can be shown that the drag is proportional to $n_{f^{\prime}} m_{r^{\prime}} / T_{f}\left(m_{f^{\prime}} / m_{\alpha} \varepsilon_{\alpha} / T_{f}\right)^{1 / 2}$. Therefore, as $x$ approaches zero the drag becomes proportional to $n_{f} T_{f}^{-3 / 2}$.

As $x$ becomes large $\phi(x)$ goes to zero and $G$ is given by

$$
G(x)_{x>2}=\frac{1}{2 x^{2}}
$$

Substituting this result into Eq. (4.4.4.1) and simplifvins shows that the $d r a g$ is proportional to $n_{f^{m}}{ }^{\alpha} / \varepsilon_{\alpha}$. Arguments for $G$ along with the drag rate $\left\langle\Delta w_{\|}\right\rangle$for alphas at $3.52 \mathrm{MeV}$ and thermal energies of $60 \mathrm{keV}$ in the hot core, $\mathrm{T}_{\mathrm{i}}=40 \mathrm{keV}, \mathrm{T}_{\mathrm{e}}=30 \mathrm{keV}, \mathrm{n}=1.6 \times 10^{14} \mathrm{~cm}^{-3}$, and surrounding halo plasme, $T_{i}=T_{e}=50 \mathrm{eV}, \mathrm{n}=5 \times 10^{12} \mathrm{~cm}^{-3}$, are given in Table 4.1 . 
TABLE 4.1

VELOCITY CHANGES DUE TO DRAG FOR A $3.52 \mathrm{MeV}$ AND $60 \mathrm{kEV}$ ALPHA IN THE REACTOR CORE AND HALO

\begin{tabular}{|c|c|c|c|c|c|c|}
\hline \multirow{2}{*}{$\begin{array}{l}\text { PI asma } \\
\text { Specie }\end{array}$} & \multirow{2}{*}{$\begin{array}{l}\text { Alpha } \\
\text { Energy }\end{array}$} & \multicolumn{2}{|c|}{ Plasme Core } & \multicolumn{3}{|c|}{ Halo } \\
\hline & & $\overline{\left(\ell_{f} w\right)}$ & $\left\langle\Delta w_{\|}\right\rangle \mathrm{m} / \mathrm{sec}$ & $\overline{\left(l_{f} w\right)}$ & $\left(\Delta \mathrm{w}_{\|}\right)$ & $\mathrm{m} / \mathrm{sec}$ \\
\hline Ions & $3.52 \mathrm{MeV}$ & 7.41 & $5.4 \times 10^{6}$ & 209.7 & 6.5 & $\times 10^{4}$ \\
\hline Electrons & $3.52 \mathrm{MeV}$ & 0.13 & $1.9 \times 1.7$ & 3.1 & 1.8 & $\times 10^{8}$ \\
\hline Ions & $60 \mathrm{keV}$ & 0.97 & $1.3 \times 10^{8}$ & 27.4 & 3.8 & $\times 10^{6}$ \\
\hline Electrons & $60 \mathrm{keV}$ & 0.02 & $3.0 \times 10^{6}$ & 0.4 & 4.8 & $\times 10^{8}$ \\
\hline
\end{tabular}

The orag rate decreases as $\mathrm{x}$ approaches zero and as $\mathrm{x}$ becomes large. Intermediate values of $x$ produce a maximum drag rate for a given plasma specie. The values of $\left\langle\Delta \omega_{\|}\right\rangle$in Table 4.1 also show that the energetic alpha drags down primarliy on the background electrons, rather than on the ions. The alpha initially drags on electrons in the not core, and ion drag becomes dominant as it slows down. Whereas in the halo, the alpha drags down on the electrons. This can be seen from Table 4.1 and Fig. 1.2.

The drag is simulated as an independent process from the angle scattering and dispersion events. At the collision sampling time, the guiding center position is determined by the appropriate azimuthal and radial drifts which have acted on the particle since the last sampling time. When the collision is sampled, the alpha orbit is first shrunk due to the drag effects between collisions. In this process the guiding center location is fixed and the particle position is moved in 
toward the guiding center along the Larnor radius vector. The change in velocity in the particle coordinate system due to drag is given by

$$
w_{\| D}=w_{\|}+\left\langle\Delta w_{\|}\right\rangle \Delta t
$$

The drag rate $\left\langle\Delta w_{\|}\right\rangle$is calculated using plasma conditions at the alpha position. The alpha position is used because the average drag rate in a nonuniform plasma may not correspond to the gulding center position. Sampling the collision process a number of times during thermalizaicion at the alpha position will produce the same results as if the drag rate were computed with the average drag rate computed across the alpha orbit.

The justification that the alpha orbit shrinks in size with the pitch angle remalning constant can be shown when we solve the equations of motion in cylindrical coordinates. This analysis assumes that there is no motion along $z$ and the electric fields are zero. If the magnetic field is in the $z$-direction and if only magnetlc and drag forces act on the particle, then Eq. (3.1.1) can be written as

$$
\begin{aligned}
& \ddot{\rho}-\rho \ddot{\phi}=\omega_{c} \rho \dot{\phi}-v \dot{\rho} \text {-direction } \\
& \left.\ddot{\rho} \div 2 \ddot{\rho} \dot{\phi}=-\omega_{c} \dot{\rho}-v_{\rho} \dot{\phi} \phi \text {-direction } .4 .8\right)
\end{aligned}
$$

The angular velocity $(\dot{\phi})$ equals $\omega_{c}$ and, since there is no acceleration in the azimuthal jirection, $\ddot{\phi}$ is zero. The rate of change of the 
Larmor radius $(\rho)$ can be found from Eq. $(4.4 .4 .9)$ and is given by

$$
\dot{\rho}=-\frac{v}{3} \rho
$$

(4.4.4.10)

The solution of Eq. $(4.4 .4 .10)$ is a decaying exponential given by

$$
\rho=\rho_{0} e^{-\frac{v}{3} t}
$$

This result is valid for uniform and non-uniform plasmas since the coordinate system is attached to the guidlng center and the guiding center drift is not predicted by this analysis. This same result car. be found by arguing that the drag decreases $w_{\|}$and transforming this change into the Laboratory system does not change the ratio $v_{1} / v_{\|}$. The pitch angle is no: affected by drag besause $\omega_{c}$ is independent of the velocity and therefore only $\rho$ decreases.

\subsubsection{Angle Scatter and Dispersion Simulation}

The collision sampling also includes angle scattering and dispersion. These velocity changes are described in the w-system of coordinates with angle scattering changing the direction of the particle velocity and dispersion changing the parallel motion. Argle scatter and dispersion are modeled as independent events where the regulting alpha direction depends on the collisional cross section and the resulting energy can elther increase or decrease. Spitzer 32 derives the angle scatter as 


$$
\left\langle\left(\Delta w_{\perp}\right)^{2}\right\rangle=\frac{A_{D}}{w}\left\{\Phi\left(l_{f} w\right)-G\left(l_{f} w\right)\right\}
$$

and dispersion as

$$
\left.\left\langle\left(\Delta w_{\|}\right)^{2}\right\rangle=\frac{A_{D}}{W} \quad G\left(l_{f} w\right)\right\}
$$

These terms can be viewed as a local standard deviation of the velocity in a collision for an alpha particle where the mean velocity change is given by Eq. (4.4.4.1). This deviation from the mean velocity is the probability that a particle can be angle scattered or dispersed around the mean during one collision (FIg. 4.5). A method of applying the Monte-Carlo technigue to Eq. $(4.4 .5 .1)$ and $(4.4 .5 .2)$ was discussed by T. Rognlien ${ }^{44}$ and is given by

$$
\begin{aligned}
& w_{\|}^{\prime}=w_{\|}+\left\langle\Delta w_{\|}\right\rangle \Delta t+\left[3\left\langle\left(\Delta w_{\|}\right)^{2}\right\rangle \Delta t\right]^{1 / 2} R_{1}, \\
& w_{1 x, y}^{\prime}=\left[1.5\left\langle\left(\Delta w_{\perp}\right)^{2}\right\rangle \Delta t\right]^{1 / 2} R_{2}, R_{3} .
\end{aligned}
$$

In these equations $\Delta t$ is the collision sampling time, $R_{1}$ are random numbers picked unlformly from 0 to 1 , but transformed by

$$
R_{i}+2\left(\frac{1}{2}-R_{i}\right)
$$




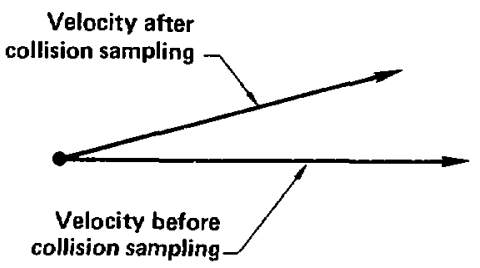

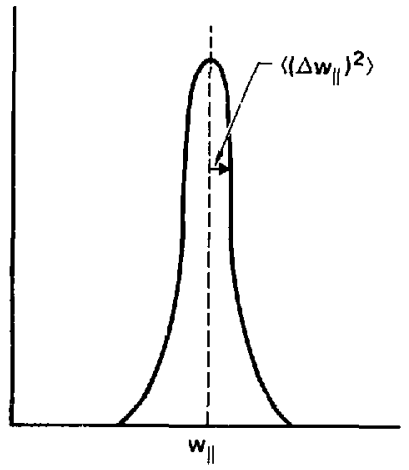

Parallel velocity probability distribution

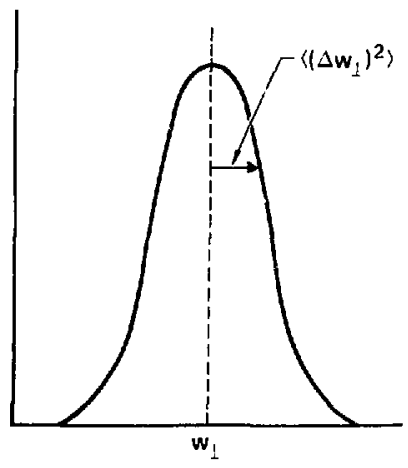

Perpendicular velocity probability distribution

Figure 4.5 Velocity changes at a collision sampling include magnitude and direction changes (A), which are determined by dispersion (B), and angle scatter, (C) about the mean velocity rinich is determined by drap 
to a uniform distribution from -1 to 1 , and the constants are necessary to obtain proper statistics.

\subsubsection{Collision Model}

Alpha particles initlally drag down in energy before large angle scattering events occur. Drag processes dominate to alpha energies of approximately $500 \mathrm{keV}$, whlch corresponds to $86 \%$ of a trapped alpha's energy being transferred to the plasma by drag. Therefore, it may be possible to retain most of the alpha energy in the plasma and then, through angle scattering losses, let the alphas escape from the trap. It is, therefore, important to model both the drag process and the angle scattering events accurately in phase space to determine the alpha energy exchange, energy deposition, velocity space losses, and radial diffusion 1osses.

As mentioned previously, the collision process is a two-step procedure. In the first step, the particle is dragged down in energy, which represents a decrease in the $w_{\|}$. Although the magnetic moment changes, the pitch and phase angle of the veloclty components remain unchanged and the particle is moved from it's present radial position to a new location along the same radius (FIg. 4.6). The new values of Larmor radius, particle position, magnetic moment, and energy can be determined by calculating $w_{\| \mathrm{D}}$ from Eq. (4.4.4.7).

After the new particle position is found, the angle scatter and dlspersion events are applied using

$$
w_{\|}^{\prime}=w_{\| D}+\left[3<\left(\Delta w_{\|}\right)^{2}>\Delta t\right]^{1 / 2} R_{1}
$$


and $\mathrm{Eq} .(4.4 .5 .4)$. In this event, the particle remajns fixed since the collision is simulated as an instantaneous event and the guiding center is allowed to move. Transforming the velocities from the w-system to the Laboratory system defines $v_{\|}$and $v_{1}$. These velocities along with the particle position are used to find a new energy, magnetic moment, guiding center position, energy, and drift velocity (Fig. 4.6). The drag results cannot be affected by the scattering and dispersion events. It is important therefore to follow a two-step collision process to ensure that these processes remain separate.

This calculation was checked by simulating a collision process in which the angle scatter and dispersion were set to zero. In this drag dominated process, the guiding center remained $f i x e d$ and the Larmor radius, $v_{\|}$, and $v_{\perp}$ chas ged to correspond to the new alpha energy. The slowing down rate has been compared with theoretical predictions, ${ }^{43}$ and the results of these comnarisons are discussed in Chapter 6 (Fig. 6.4).

Angle scattering results have been checked by following an alpha close to thermal energies and determining if final plasma average energy is $3 / 2 \mathrm{~T} e$. That is when a $40 \mathrm{keV}$ alpha was started in a plasma with uniform ion and electron temperatures of $40 \mathrm{keV}$ and when there are no loss mechanisms, then the average alpha energy becomes $60 \mathrm{kev}$, which is expected. These checks verified the accuracy of the angle scatter and collision operators.

The impiementation of this simulation into a working code is shown by the block diagram of FIg. 4.7. The guiding center position is 

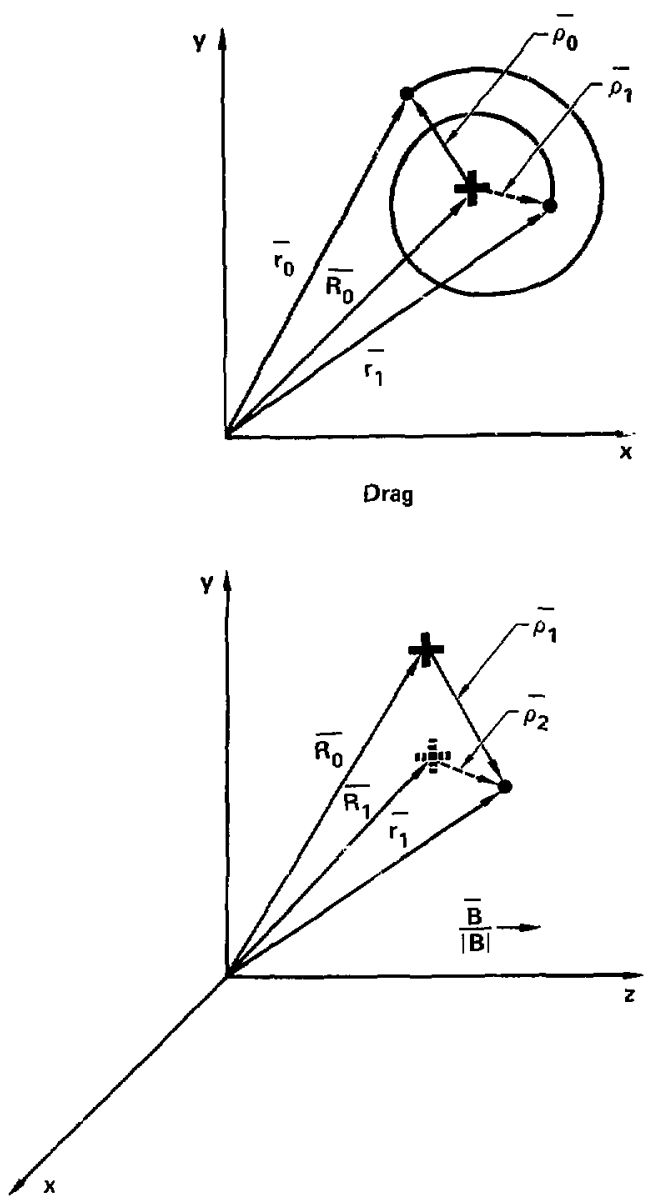

Angle scatter and dispersion

Figure 4.6 The two step collision process first reduces the Larmor radius because of drag (A) and then incorporates angle scatter and dispersion (B) 


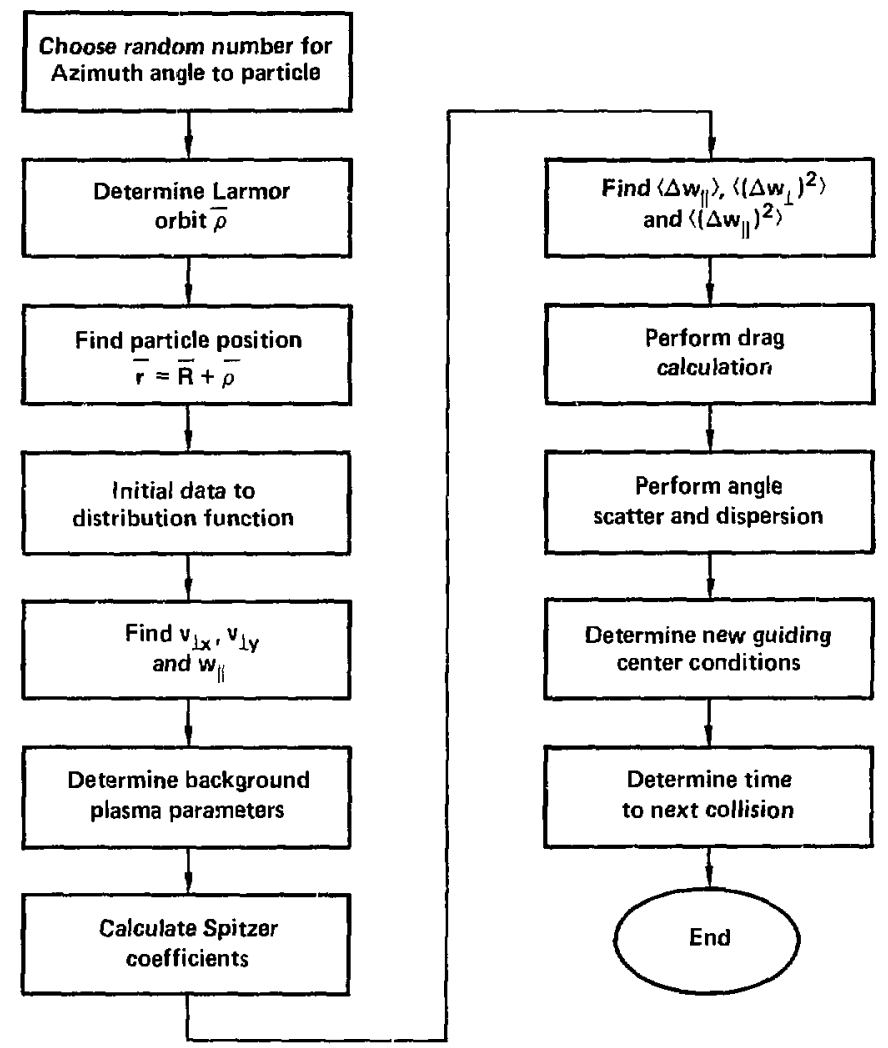

Figure 4.7 Block diagram of the collision subroutine used in the Monte-Carlo simulation 
known, so the initial step determines the particle position and velocities in the Laboratory and $w$-system. In the next step, the local density and temperature is determined at the particle position which is needed as input into the Spitzer collision terms. The change in particle velocity due to drag is computed using Eq. (4.4.4.7), and a new particle position, magnetic moment, energy, etc., are calculated. The angle scatter and dispersion terms are then calculated using the particle position found in the drag step. This step leads to a new guiding center location, Larmor radius, magnetic moment, and energy. Finally, a new collision interval is calculated. During the collision calculation certain valikes are stored for future analysis. The velocity distribution functión requires the parallel and perpendicular velocities before and after the collision. The energy change of the alpha for both the drag, and scattering process is also needed. The energy given to lons and electrons is stored along with particle position information at each collision. This information is tabulated and stored for plotting and statistical analysis. 


\section{PART 5}

\section{PLASMA DENSITY AND TEMPERATURE PROFILES AND DRAG DRIFT}

\subsection{Introduction}

Plasma radial density and temperature profiles are specified at the plasma midplane and are made consigtent with profiles from present reactor studies. These profiles must be continuous in $r$ since the radial drift velocity depends on $d v / d r$ where $v$ is the drag rate defined by $\left\langle\Delta w_{\|}\right\rangle / w$ and $\left\langle\Delta w_{\|}\right\rangle$is dens1ty and temperature dependent. Even though the radial drift rate is profile dependent, relevant plasma design Information can be obtalned from the general form of $d v / d r$ and by studying several drag profiles.

\subsection{Density Prof $11 \mathrm{e}$}

Radial density profiles can be modeled by either Gaussian, parabolic, quartic, or higher power curves. The quartic prcfile is used in many reactor designs because 1 t is flatter in the piasma core region, which produces higher fusion reaction rates. These density profiles are modeled by

$$
n(r)=n_{0}\left[1-\left(\frac{r}{r_{p}}\right)^{\gamma}\right]
$$

where $r_{p}$ is the plasma radius, $n_{0}$ is the on-axis density, and $Y$ can take on any value. The power profiles fit experimental data near the axis, but they are zero when $r$ equals $r_{p}$ and become negative when 
$r>r_{p}$. Values of $n(r)$ for $r>r_{p}$ are therefore defined by another equation. One way of modeling $n(r)$ is to define

$$
\begin{array}{ll}
n(r)=n_{0}^{*}\left[1-\left(\frac{r}{r_{p}}\right)^{\gamma}\right]+n_{n}\left(\frac{r}{r_{p}}\right)^{\gamma} ; r \leq r_{p} \\
n(r)=n_{n} ; & r>r_{p}
\end{array}
$$

where $n_{0}^{*}$ is equal to $n_{0}+n_{h}$. In this model there is a discontinuity In the slope of the density at $r_{p}$.

A Gaussian profile can also be used to model the density profile, and it does not have a discontinuous slope at the plasma edge. The Gaussian, however, does not accurately model the halo region where the density is approximately constant, because $n(r)$ continues to decrease for $r>r_{p}$. The halo can be modeled by adding a flat density profile to the generalized form of the Gaussian given by

$$
n(r)=A \exp \left[-x^{Y}\right]+B
$$

The value of $x$, which is proportional to $r / r_{p}$, and $Y$ are chosen so that $n(0)$ is the on-axis density and $n\left(r_{p}\right)$ is the halo density, which is several orders of magnitude smaller than $n(0)$. Th1s Gaussian shape is very peaked and narrow, which is not consistent with present reactor design density profiles. Therefore, it is difficult to satisfy all the necessary conditions for the density profile using the simple Gaussian of $\mathrm{Eq} .(5,2,3)$. 
The density profile model that is used is similar to Eq. (5.2.3) for the core region, but it has a smooth transition at $r=r_{p}$ where the density profile is flat and becomes equal to the halo density. The general form of this model is given by

$$
\begin{array}{ll}
n(r)=\left(n_{0}-n_{h}\right)\left\{1-\exp \left[-\left(\frac{1-\frac{r}{r_{p D}}}{1-r_{D}}\right)^{Y}\right]\right\}+n_{n} ; r \leq r_{p} \\
n(r)=n_{h} ; & r>r_{p}
\end{array}
$$

The density fails of at a rate determined by a scale length $r_{p D}$ and an arbitrary function $\left(1-f_{D}\right)$ where $f_{D}$ is less than one. The value of $Y_{D}$ is greater than one and also determines the profile shape. When $Y_{D}$ equals 1.15 and $f_{D}$ is 0.75 , the density profile is very similar to a quartic profile, as shown in Fig. 5.1. The advantage of using Eq. $(5.2 .5)$ is that the first and second derivatives of the density are zero when $r$ equals $r p$. Therefore, this profile eliminates the discontinuity at the plasma halo interface and malntains core and halo density profiles consistent with present models.

\subsection{Temperature Profiles}

The temperature profile is modeled using the same functional form as Eq. $(5.2 .5)$ and is given as 


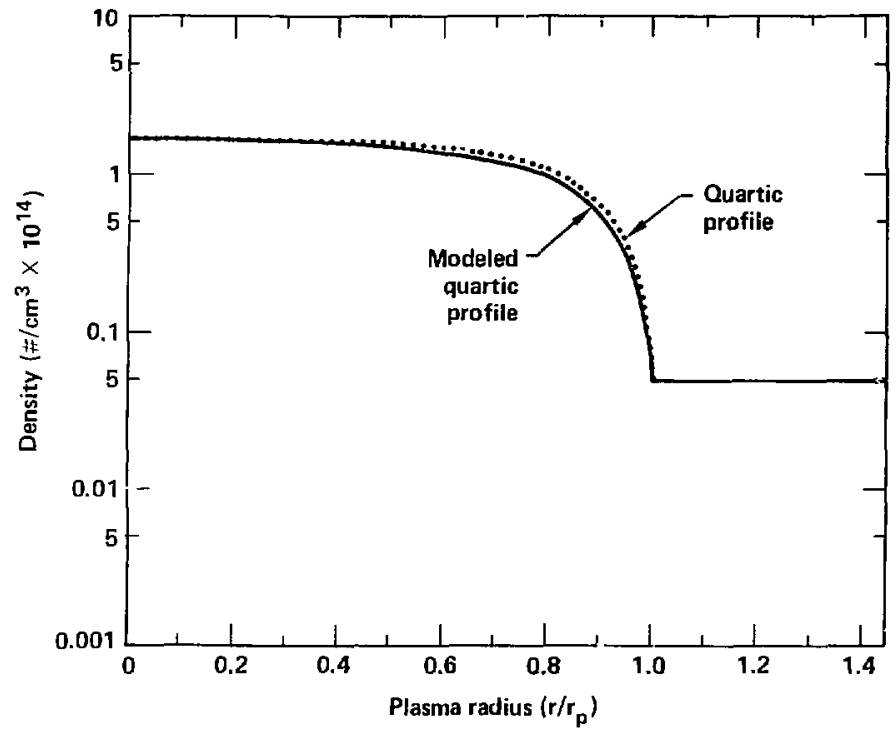

Figure 5.1 Quarije density profile and the modeled quartic profile used in the Monte-Carlo calculation for the generic reactor destgn 


$$
\begin{aligned}
& T_{i}=T_{o i}\left\{1-\exp \left[-\left(\frac{1-\frac{r}{r_{p T}}}{1-\hat{r}_{T}}\right)^{\gamma_{T}}\right]\right\}+T_{h} ; r \leq r_{p T} \\
& T_{1}=T_{h} ; \quad r>r_{p T}
\end{aligned}
$$

where $\mathrm{T}_{01}$ is the on axis ion or electron temperature. The exact temperature profile is unknown; therefore, the constants $r_{\mathrm{pT}}, f_{\mathrm{T}}$, and $r_{T}$ can be chosen arbitrarily. We initially chose $r_{p T}$ to equal $r_{p D}$ because during initial plasma operation the temperature $\left(r_{p T}\right)$ and density $\left(r_{p D}\right)$ scale lengths should equal $r_{p^{*}}$ This scale length relation implies that the plasma temperature and density become equal to the constant halo values at the edge of the plasna. We also know from present experimental data that the plasma temperature has a flatter core profile than the density profile. It is expected that this condition will persist in reactor plasmas; therefore, $\gamma_{T}$ and $f_{T}$ are chosen so that the temperature profile is flatter than the density profile in the plasma core. These profiles are also chosen so that the gradient of the drag force across the alpha orbit is reasonable for alpha adiabaticity considerations. 39 Although small temperature gradients across an alpha orbit are desirable, this is not possible since plasma temperatures vary by three orderg of magnitude over an approximate $50-\mathrm{cm}$ radius. The drag gradient is therefore limited to occur over $30 \%$ length of an alpha Larmor radius when the beta corrected $p$ is calculated with $\epsilon_{\alpha}=3.52 \mathrm{MeV}$. The alpha is assumed to be adiabatic because the axial fields are designed for mimror confinerient, 
the value of $r_{p} / \rho$ is on the order of unity, and the density and temperature gradients occur over a relatively large fraction of a Larmor orbit.

The temperature and density profiles must be chosen so that a plasma temperature greater than $10 \mathrm{keV}$ coupled w1th high plasma density is malntained over a significant portion of the core plasma. The high density and temperatures are needed to ignite the fusion reaction. Because of these considerations, the value of $\gamma_{T}$ was selected to be 3.0 and $f_{T}$ to be 0.75 for the reactor designs used in this study. The corresponding temperature profile along with the density profile is shown in Fig. 5.2 .

The temperature and density profiles may not keep the same scale length characteristics, i.e., $r_{p D} \neq r_{p T}$. For example, if a large fraction of alpha energy is deposited in the halo because of the halo electron drag, then this will shift the thermal distribution of alphas to be weighted toward the plasma-halo boundary and the alpha energy will be concentrated in this region. This heating will Increase the ion and electron temperature in the halo and therefore cause a shift in the scale lengths so that $r_{\mathrm{pD}}$ may not equal $r_{\mathrm{pT}}$. When $r_{\mathrm{pD}} \neq \mathrm{r}_{\mathrm{pT}}$, this is referred to as the two-scale-length problem. An example of the twoscale-length density and temperature profiles is shown in Fig. 5.9.

5.4 Drag Drift

The radial drag gradient is given by 


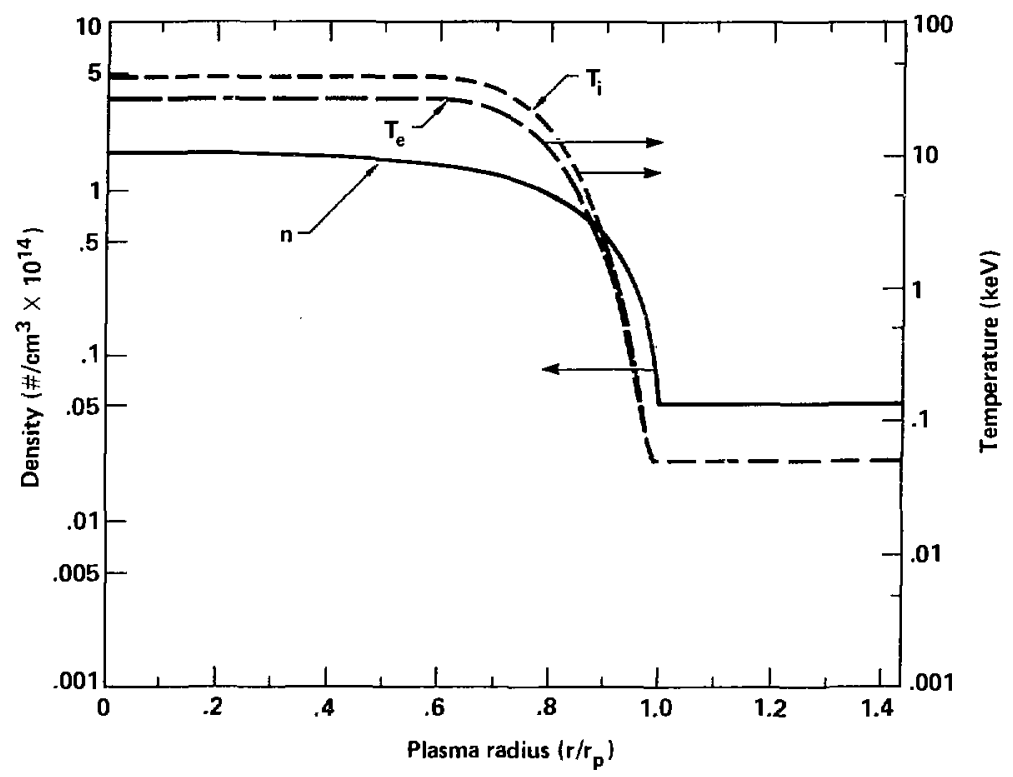

Figure 5.2 Modeled density and temperature profiles for the single-scale-length generic reactor design used for the Monte-Carlo simulation 


$$
\frac{d v}{d r}=A_{v}\left\{\left[\phi(x)-x \Phi^{\prime}(x)\right] \frac{d n_{f}}{d r}-\frac{n_{f}}{T_{f}} x^{3} \phi^{\prime}(x) \frac{d T_{f}}{d r}\right\}
$$

where $A_{V}$ is a constant for a given alpha energy and is given by

$$
A_{v}=\frac{8 \pi q^{4} z_{f}{ }^{2} z^{2} L n \Lambda_{f}}{2{ }_{o}^{2} m_{\alpha} w^{3}}\left(1+\frac{m_{\alpha}}{m_{f}}\right) .
$$

There is no drag drift when $d v / d r$ is zero, and this occurs whenever the term enclosed in square brackets 19 zero. It is readily apparent that this condltion is satisfied whenever $\mathrm{dn}_{\mathrm{f}} / \mathrm{dr}=\mathrm{dT}_{\mathrm{f}} / \mathrm{dr}=0$, which occurs on the plasma axis due to symmetry conditions and in the halo where the Censity and temperature are constant. However, the general condition for zero drag drift is given by

$$
\frac{d n_{f} / n_{f}}{d T_{f} T_{f}}=\frac{x^{3} \phi^{\prime}(x)}{\Phi(x)-x \Phi^{\prime}(x)}
$$

where the left side is the ratio of the density-to-temperature scale lengths and the right side is determined by the error function, its derivative, and the ratio of alpha speed to plasma thermal speed given by $x$. It is highly unlikely that the plasma density and temperature profiles will relax to a condition in which Eq. (5.4.3) is satisfied everywhere. Therefore, we expect $d v / d r$ to take on positive or negative values, and this will lead to alpha particle radial drag drifts. 
It has been argued that there will be at least two reglons along the plasma radius where the drag drift is zero. The number of radial locations with $d v / d r=0$ can be determined by solving Eq. (5.4.3) for the zero points or by plotting the left and right side of Eq. (5.4.3) as a function of $r$. In elther method, it can be shown that the right side of this equation is well behaved with limits of 1.5 as $x$ goes to zero and zero as $x$ goes to infinity. The left side of the equation is not well behaved, being undefined at $r=0$ and $r \geq r_{p}$. The density and temperature profiles, although smooth, determine whether there are two or more radial locations with $d v / d r=0$.

The fact that there can be more than two radial locations of zero drag drift is important because this implies that there are different alpha radial drift zones within the plasma. For example, the drag profile for Fig. 5.2 is shown in FiB. 5.3, and the drag gradient is shown in Fig. 5.4. These two profiles show that there are three drag drift regions inside this piasma. That is, when $r / r_{p}<0.63$ and for $0.97 \leq r / r_{p}<1.0$, the value of $d v / d r$ is negative and the radial drift is inward. When $0.63 \leq r / r_{p} \leq 0.97$, the value of $d v / d r$ is positive and the drift is outward. There 1s, therefore, a possibllity of having radial zones that are rioh in themmal alphas and other zones that are poor in thermal alphas. If the radial drift for given plasma proflles causes an excessive separation of thermal alpha particles from one radial zone to another, then the inftial temperature and density profiles produce a nonequilibrium alpha distribution. The plasma density and temperature profile in this case will relax so that the 


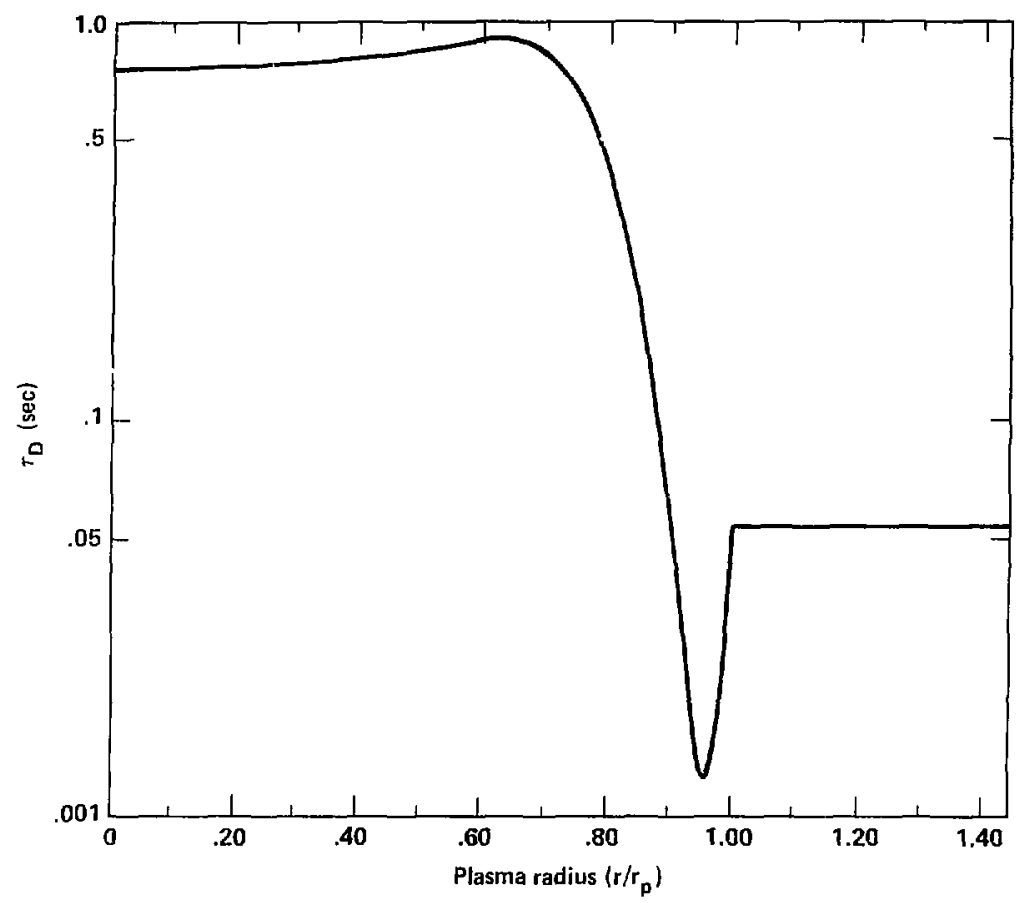

Figure 5.3 Drag profile for the density and temperature profiles of Fig. 5.2 


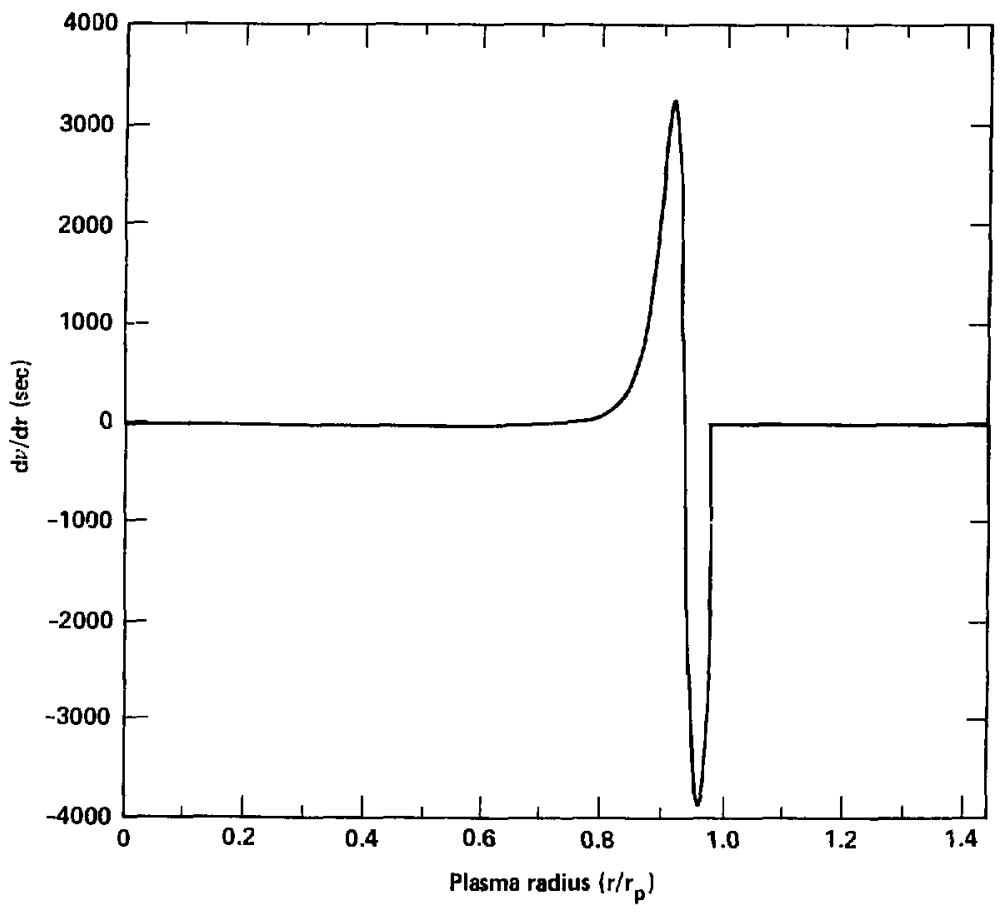

Eigure 5.4 Drag gradient for the drag profile of Fig. 5.3 
plasma ions, electrons, and the thermal alphas are in an equiliorium distribution.

The relaxed plasma profiles cannot be obtained selfconsistently, since the problem is too complex. Therefore, an 1terative process is used in which density and temperature profiles consistent with present reactor designs are used to generate drag and drag gradient profiles. For example, the quartic density profile and step temperature profile shown in Fig. 5.5 are used in many reactor studies. Since the step temperature profile leads to a discontinuity in $d v / d r$, the step can be modeled using Eq. (5.3.1) with a continuous curve as shown in Fig. 5.6. This profile produces the drag and drag gradient profiles of Figs. 5.7 and 5.8, respectively. The sharp temperature boundary of Fig. 5.6 produces a large drug over a small radial region. In this case we felt that the sharp temperature boundary could not be maintained by the plasma and the temperature profile was relaxed to the profile shown in Fig. 5.2. These profiles with equal density and temperature scale lengths were then used to evaluate alpha transport in a tandem reactor (see chapter 6).

The essential results of these alpha transport studies can be related to the drag drift, which is a function of the drag gradient profile of Fig. 5.4. In these studies, the drag drift in the varlous radial zones across the plasma caused a large fraction of alphas to thermalize at the plasma edge. This nonequilibrium distribution would eventually relax and cause the temperature and denslty scale lengths to change. This observation lead to the two-scale-length concept in which 


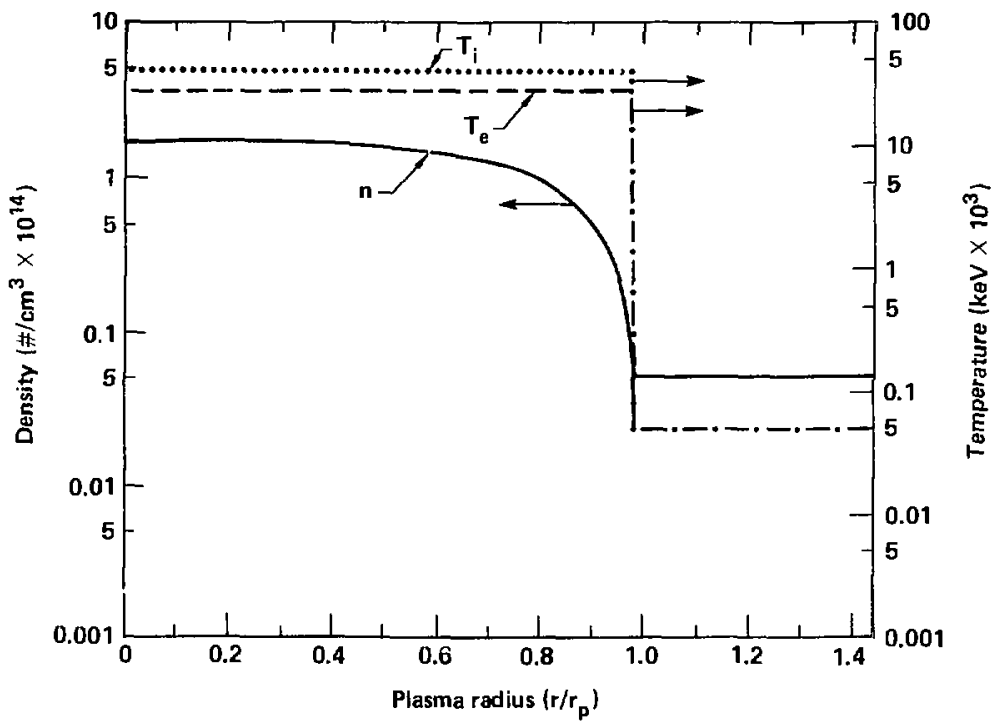

Figure 5.5 Quartio density and step temperature profile used for some reactor designs 


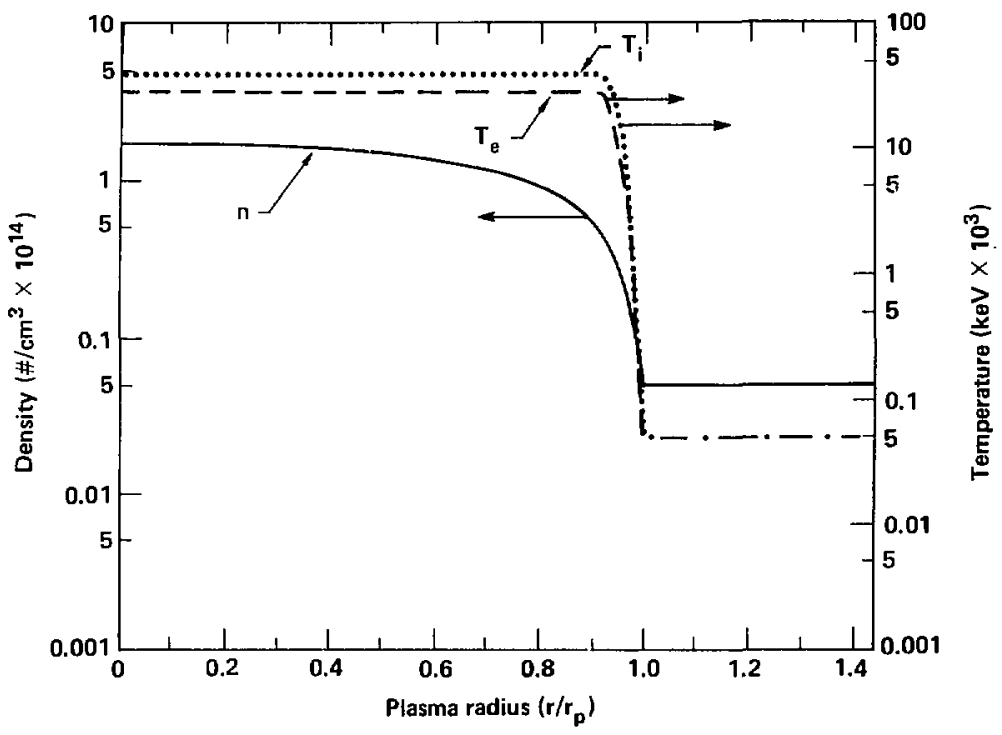

Figure 5.6 Modeled quartic density and it, ?p-temperature profile using Eq. 5.2.4 and 5.3.2, respectively 


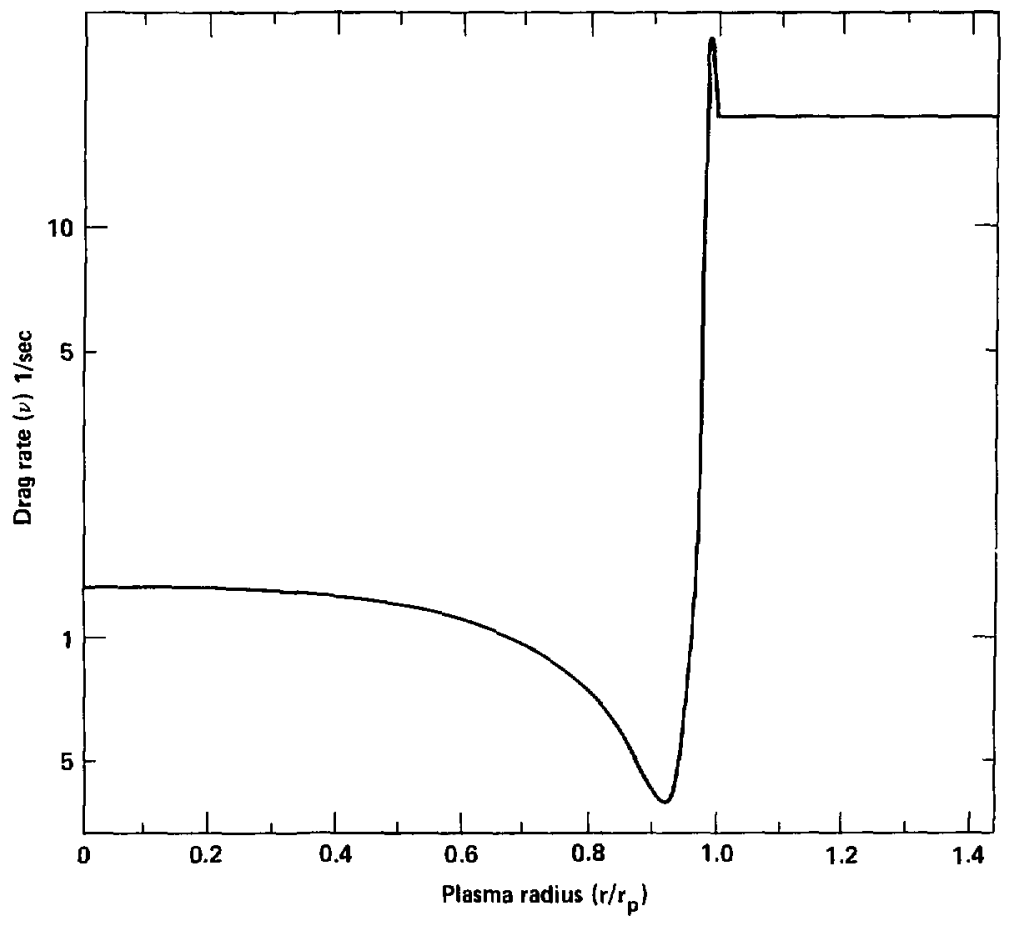

Figure 5.7 Drag profile for the density and temperature profiles of $\mathrm{Flg} .5 .6$ 


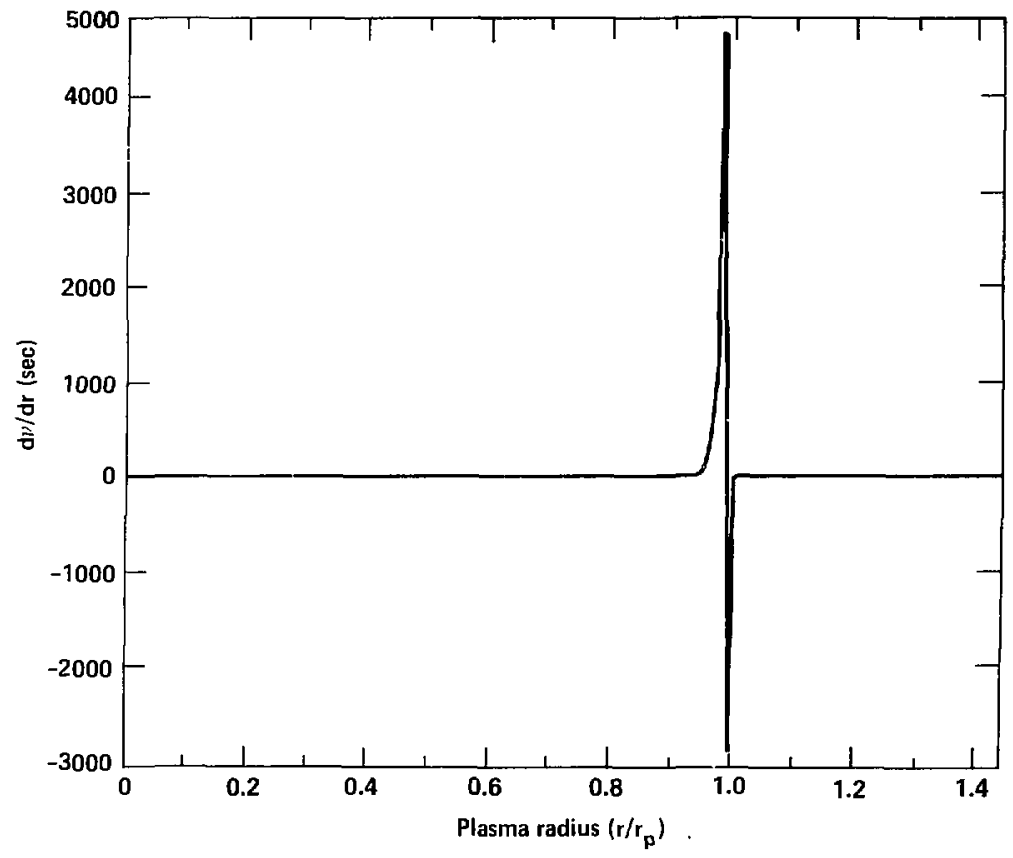

Figure 5.8 Drag gradient for the drag profile of Fig. 5.7 
the quartic density profile remained unchanged with the plasma density becoming equal to the halo density at $r_{p}$. The temperature scale length, however, was increased to correspond to a flatter temperature profile in the reactor core $\left(r<r_{p}\right)$. By adjusting $r_{p T} / r_{p D}$ to take on yalues greater than one, we can adjust the relation between the density and temperature profiles without changing the plasma axis parameters. An example of the two-scale-length density and temperature profiles is shown in Fig. 5.9. The assoclated drag and drag gradient profile is shown in Figs. 5.10 and 5.11. The drag gradient of Fig. 5.10 is significantly different from that of Fig. 5.4 in that the extreme values of du/dr for the two-scale length problem are approximately five times smaller than that of the single-scale-length problem. This implies that the radial drag orift is much smaller for the two-scalelength problem. The next difference is that the largz positive drift in the two-scale-length pi iblem occurs for $r>r_{p}$. Therefore only those alphas that have orbits extending beyond $r_{p}$ wll have large positive radial drifts. Because of these observations, we can expect thermal alpha distributions that are closer to equilibrium from the two-scale-length profiles.

\subsection{Conclusion}

We have shown that continuous radial density and temperature profiles can be modeled using Eq. $(5.2 .5)$ and $(5.3 .1)$. These profiles are used to determine the radial drag and drag gradient profiles across the plasma. The drag gradient is used to determine the alpha transport and appropriate changes to the density, and temperature profiles are 


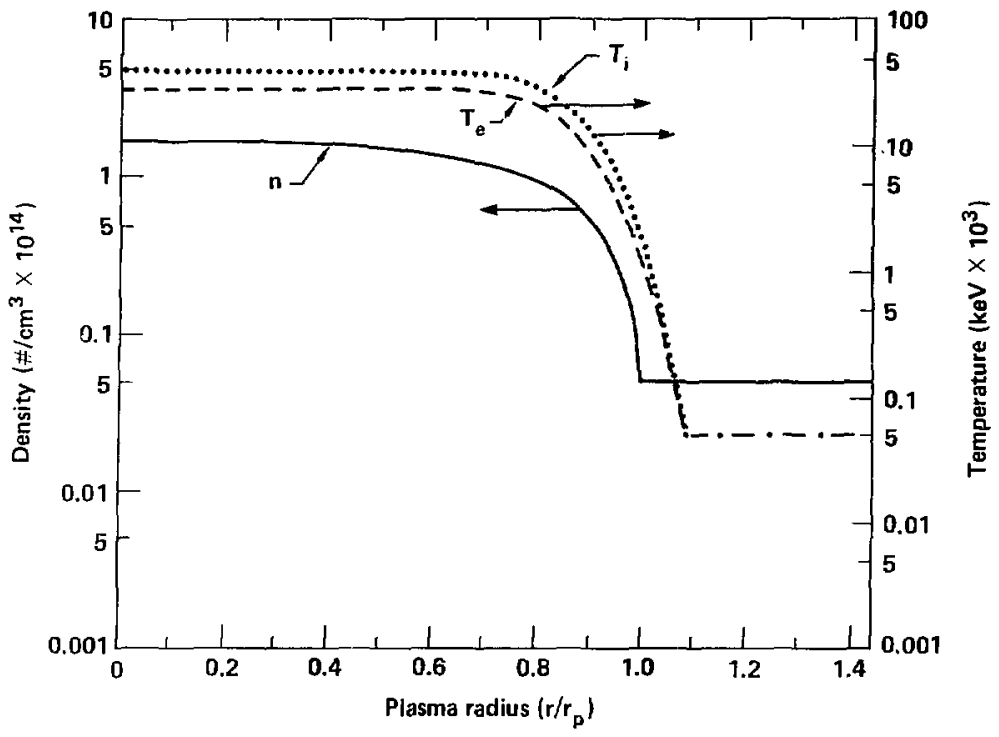

Figure 5.9 Density and temperature profile for the two-scalelength problem 


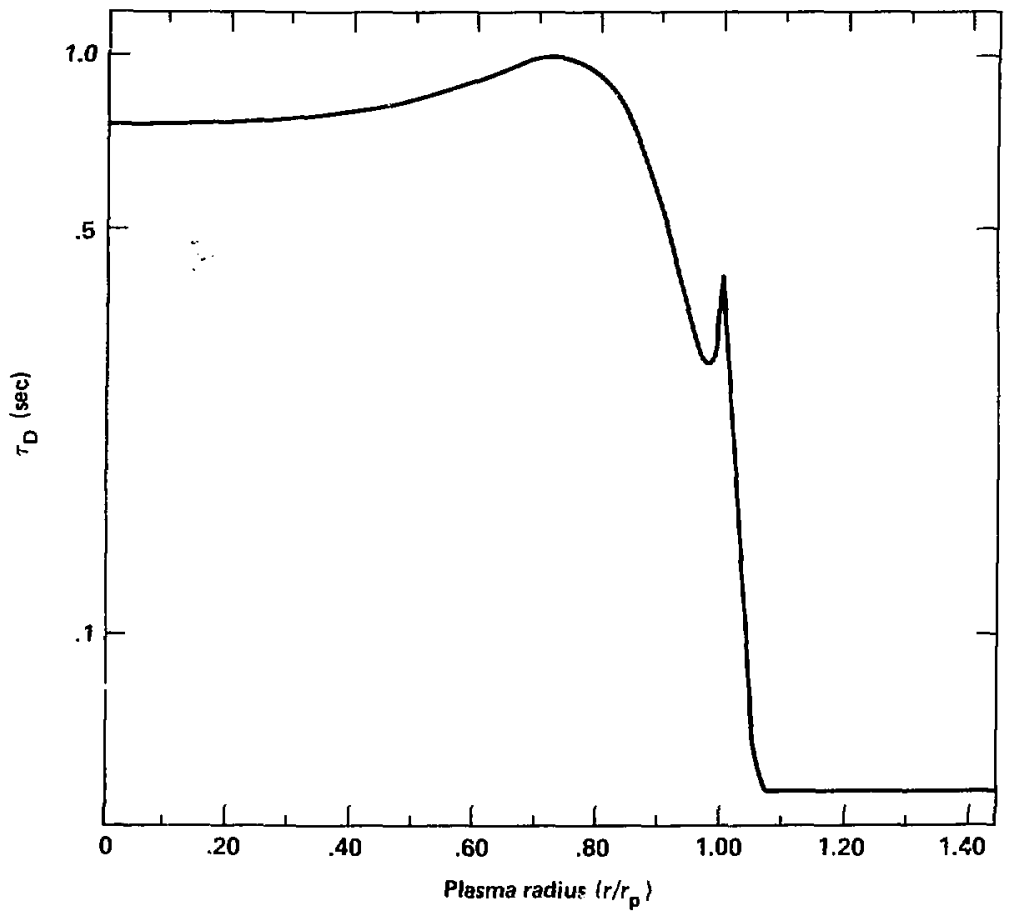

Figure 5.10 Drag profile for the density and temperature profiles of F'ig. 5.9 




Figure 5.11 Drag gradient for the drag profile of Fig. 5.10 
made when alpha transport calculations lead to nonequilibrium distributions. 


\section{PART 6}

\section{ALPHA PARTICLE THERMALIZATION STUDIES}

\subsection{Introduction}

The preceeding chapters described the ingredients needed to study alpha particle transport in tandem mirror reactors. These Ingredients were used to produce steady state information about the alpha thermalization process. Because there are many ways errors can be mixed into the calculation with the ingredients, there are several diagnostics which are used to verify the accuracy of the computed results. In the following section, the major diagnostics which are included as part of the calculation are discussed.

After discussing the diaghostics, a description of alpha particle thermalization results for the generic reactor design are presented. This includes a description of the alpha thermalization rates, along with energy deposition profiles and alpha power distribution profiles. The technique of converting the Monte-carlo sums in radial bins to thermalization rates and power profiles is discussed. Pertinent statistical information is also presented. The generic reactor results are too narrow and specific to generate generalized reactor design criteria. As shown in Chapter 5, the radial orift rate is dependent upon the plasma temperature and density profiles. Changes in the magnetic field $\left(B_{v}\right)$, plasma radius $\left(r_{p}\right)$, and $B$ can also affect the alpha thermalization process. In order to systematically study these effents, a heurlstic argument is given in 
which the alpha energy deposition depends on $\frac{\rho}{r_{p}}$. This parameter can be varled for a given density and temperature proflles by yarying $r_{p}$ ' peak beta $(\hat{\beta})$, or $B_{v}$. The results of varying $\frac{\rho}{r_{p}}$ for the generic density and temperature profiles are presented. A small perturbation in the density profile changes the value of $\frac{\rho}{r_{p}}$, and this effect, along with its implications, is discussed.

The single-scale-length temperature and density profiles limit the average plasma beta $\langle\beta\rangle$ to low values. Separating the scale lengths increases $\langle\beta\rangle$ and allows for more flexplilty in studying temperature and density profile effects on alpha confinement. Results of varying these profiles and their associated scale lengths are also presented.

\subsection{Calculation Yertification}

The calculations have been verffied by including several diagnostics within the Monte Carlo slmulation. Plots of energy decay of individual alpha particles as a function of time are made and compared with theory. Also included in this diagnostic is the fraction of alpha energy given to plasma electrons and ions. A second diagnostic is the velocity space distrlbution which shows velocity space information as the alpha particles slow to thermal energies. The velocity distribution is used to verify the slowing in distribution of alphas and to verify the loss cone calculation. The alpha energy distribution function is also computed, and the results are plotted and 
compared with theory. Plots of alpha radial position as a function of the number of collision sampling events are also generated.

\subsubsection{Alpha Energy Decay}

Alpha particles slow to thermal energies by transferring energy to plasma ions and electrons. Alpha velocity changes and the corresponding energy changes $(\Delta E)$ are calculated at every collision sampling. The total velocity change is made up of contributions from alpha-ion and alpha-electron interactions, which are computed individually. These individual velocity changes are then used to calculate the alpha energy given to plasma ions and electrons, which is used as a diagnostic tool.

The collision model is applied at discrete times, and the velocity changes are randomized by the Monte-Carlo technique. The alpha energy given to plasma lons $\left(\Delta \varepsilon_{1}\right)$ and electrons $\left(\Delta \varepsilon_{e}\right)$, divided by the total alpha energy, can be plotted as a function of time for individual alpha particles. We can compare these Monte-Carlo results with slowing down theory which has been derived by several authors. $7,43,45,46$ In these derivations, the average rate of alpha enengy exchange with plasma ions and electrons is determined using appropriate drag cross-sections. We use the theoretical energy loss rate derived by Sivukhin 43 and given by

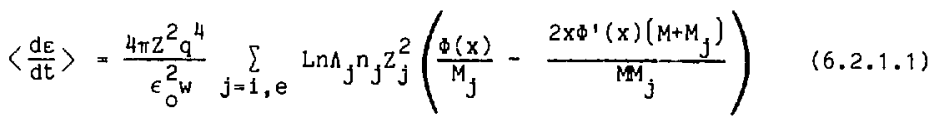


where the subsoript $\mathrm{J}$ refers to ef ther the electron or ton species. In Eq. (6.2.1.1), the background plasma density and temperature are assumed constant, and the energy change $(\Delta E)$ can be found by a simple Euler integration scheme when $\Delta t$ is small. The result of this integration determines the total amount of alpha energy given to the plasma. Each $\Delta t$ interval also determines the incremental amount of alpha energy that goes to the plasma lons and electrons, and this Information ia used to plot the theoretical values of $\frac{\Delta E_{1}}{E_{\alpha}}$ and $\frac{\Delta E_{e}}{E_{\alpha}}$ as a function of time.

The comparison between the Monte-Carlo and theoretical energy loss is shown in Fig. 6-1(a) through (d). A perfect agreement between the Monte-Carlo simulation and theory cannc be expected. The difference occurs because the theory only inwudes drag in the collision model and assumes that angle scatter and disporsion are negliglble until the alpha energy is less than $15 \mathrm{Te}$. This, however, does not mean that angle scatter and dispersion can be neglected at higher energies, and when they are included, the individual alpha energy loss w1ll vary from theory. Some alphas decay more slowly than theory [Fig. 6-!(a)] suffering up-scattering in energy as the alpha decays to thermal energles, whlle others decay faster [Fig. 6-1(b)], and st11l others compare favorably with theory [F1g. 6-1(c)].

Both the theory and the Monte-Carlo polnts in Fig. 6.1 (a through c) were generated in a uniform plasma with constant density and temperature. In general, the plasma is not uniform, and alphas which sample the halo see a large variation in background plasma temperatures 
(a)

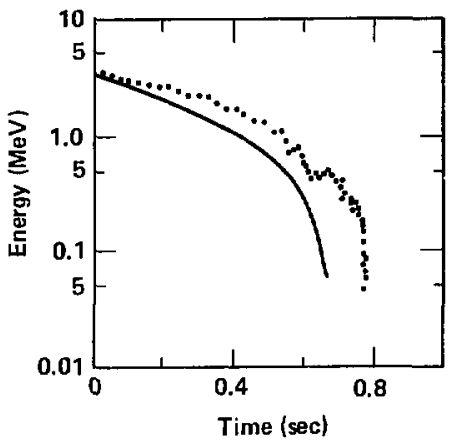

(c)

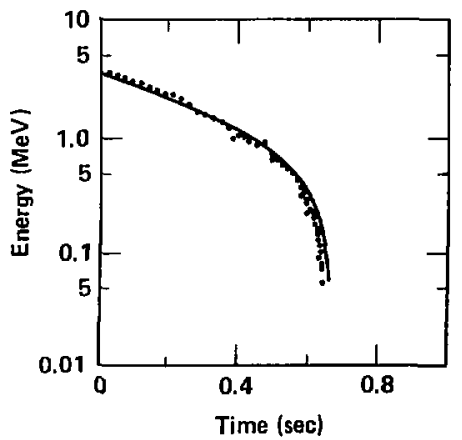

(b)

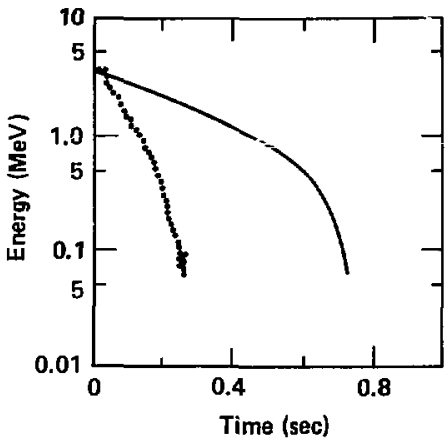

(d)

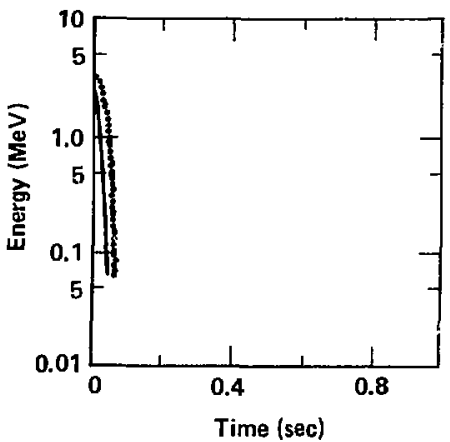

Figure 6.1 Alpha particle energy decay in the hot reacting core plasma(a-c) and in the halo(d). Solld curve is a theoretical prediction given by Sivukhin 43 
and densities. The energy decay for an alpha that samples the halo is approxlmately ten times faster than alpha energy decay in the core plasma, as shown in F1g. 6-1(d). Alphas that sample the halo drag down primarily on halo electrons and, as a consequence, more alpha energy is transferred to electrons than uniform plasma theory predicts.

Therefore, the theoretical predictions for $\frac{\Delta \varepsilon_{e}}{\varepsilon_{\alpha}}$ and $\frac{\Delta \varepsilon_{i}}{\varepsilon_{\alpha}}$ represent limfting valueg for alpha energy transfer in actual plasmas.

Although individual alphas w11l not always agree with theory, it is expected that the average energy given to the lons and electrons from all the alphas will match theoretical predictions. This numerical experiment has been performed, and the average energy to the plasma ions and electrons calculated from the code differs from Sivukhin's theory by less than $10 \%$ at plasma temperatures of $60 \mathrm{keV}$ and less than $4 \%$ at plasma temperatures of $5 \mathrm{keV}$ for 1000 alpha particles. A comparison betweer the calculation's average fraction to lons $\left(\frac{\Delta \varepsilon_{i}}{\varepsilon_{\alpha}}\right)$ and to electrons $\left(\frac{\Delta E_{e}}{E_{\alpha}}\right)$ as a function of electron temperature is shown in F1g. 6.2, where the solid 1 ine is derived from Slvukhin. We can compare the solid curve in FIg. 6.2 with the Rose approximation of Fig. 1.2. The difference between these curves occurs because Rose uses an approximation to the error function, whereas Sivukhin does not. The Monte-Carlo algorlthm uses Spitzer's collision model, which can be used as a starting polnt to dem'. Eq. $(6.2 .1 .1)$; therefore, we expect to have good agreement betw + $t$ Monte-Carlo results and the theoretical 


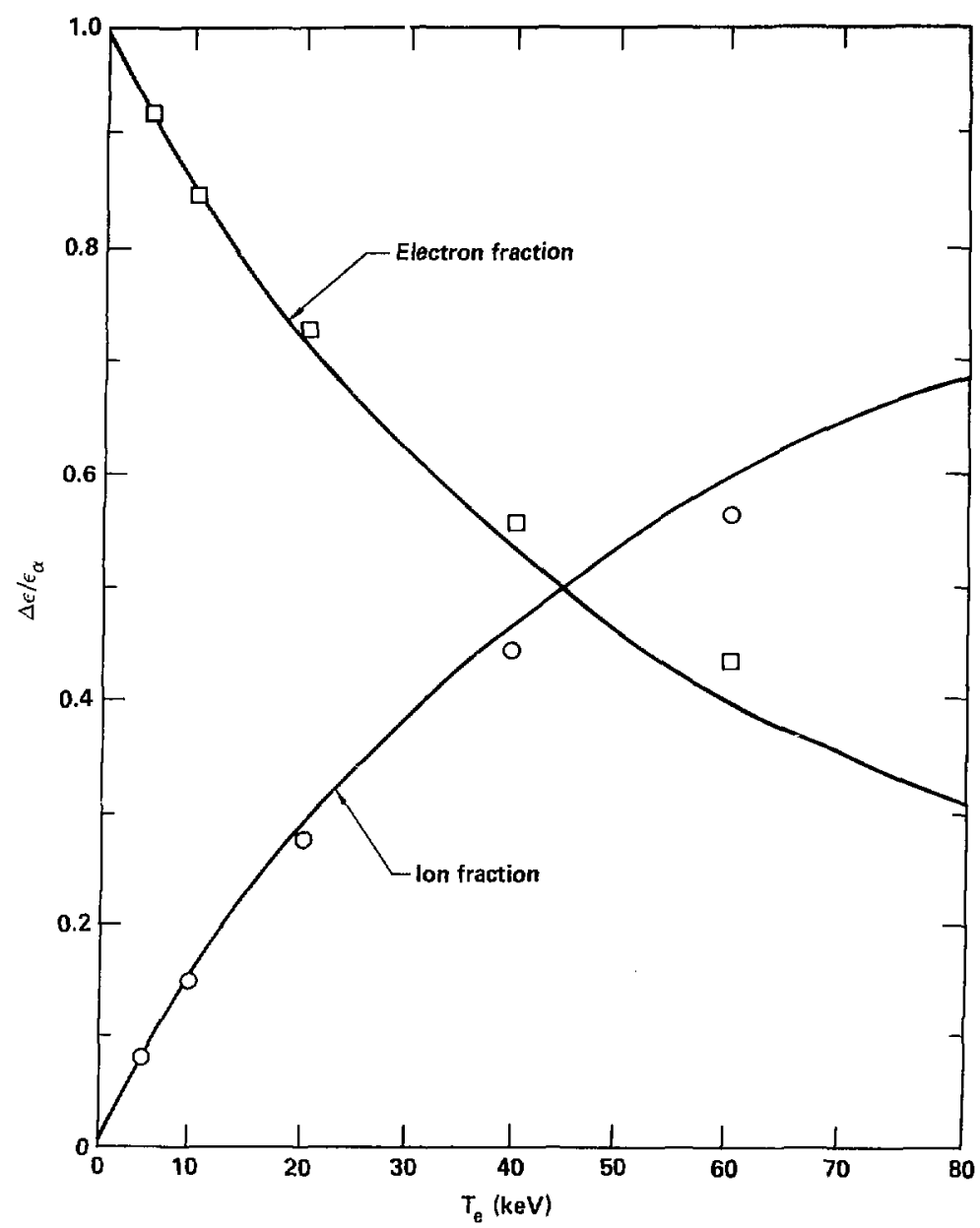

Figure 6.2 Fraction of alpha energy given to lons(o) and electrons (1) as a function of plasma electron temperature and compared with Sivukhin ${ }^{43}(-)$ 
results predicted by Sivukhin. The small difference between the MonteCarlo slmulation and theory occurs because we include angle scatter and dispersion in the simulation, we use a low-order intagration scheme to integrate Eq. (6.2.1.1), and the Monte-Carlo simulation produces an approximation to the exact fractional values.

\subsubsection{Velocity Distribution Function}

The velocity distribution function $f(\bar{r}, \bar{v})$, defines the steady-state phase space distribution of particles. The particle densities can be obtained by integrating the velocity distribution function as

$$
n(\bar{r})=\int_{\bar{v}} f(\bar{r}, \bar{v}) d \bar{v}
$$

where the velocities are glven in cylindrical coordinates by $v_{1}$ and $v_{\|}$. In order to find the distribution function, we divide velocity space into small volumes, with dinensions $\Delta v_{\|}, \Delta v_{1}$, and $\Delta \Theta$. The alpha distribution is isotropic in $\theta$, and when $\Delta v_{\|}$and $\Delta v_{\perp}$ are small, the distribution function is constant in the area $\Delta v_{\|} \times \Delta v_{1}$. Then Eq. $(6.2 .2 .1)$ can be approximated over $\Delta v_{\|}$and $\Delta v_{\perp}$ by

$$
n(1, j)=2 \pi f(i, j) v_{1 j} \Delta v_{1} \Delta v_{\|}
$$

The $i$ and $j$ refer to a bin location in velocity space where 1 corresponds to the $x$ or $v_{\|}$direction and $j$ is in the $v_{\perp}$ direction. The total particle density (N) is found by summing the $n(i, j)$ densities as 


$$
N=\sum_{1} \sum_{j} n(1, j)
$$

Next, the normalized distribution is found from

$$
\hat{f}(1, j)=\frac{1}{\pi^{2} v_{\perp j} \Delta v_{\perp} \Delta v_{\|}} \frac{n(1, j)}{N}
$$

where the ratio $\frac{n(i, j)}{N}$ is assumed to be proportional to the amount of time the alpha spends in each $b 1 n, 1 . e . \frac{\Delta t(1, j)}{\Delta T}$ and $\Delta T$ is the total time required to follow all the alphas from blrth to thermallzation. The calculation of $\Delta t(i, j)$ for one particle can be found by knowing the time between sampling a collision event and the velocity space coordinates before and after the sampling. This information, shown schematicallv in Fig. 6.3, is used to mark the end points of the alpla's velocity space trajectory. This trajectory is assumed to be linear because the sampling time is small compared to the alpha drag and $90^{\circ}$ scattering time. Thls assumption allows us to use linear interpolation to deternine the amount of time the particle spends in each velocity bin. The total value of $\Delta t(1, j)$ is found by suming the contrioutions from all alpha trajectories that pass through bin $(i, j)$ as they slow down. The value of $\Delta t(i, j)$ is associated with the coordinates at the center of the bin.

A velocity distribution function is actually associated with every point in plasma velocity space. In determining $f\left(\bar{r}_{1} v_{\|}, v_{1}\right)$ particle information is generated at many radial locations, but there 


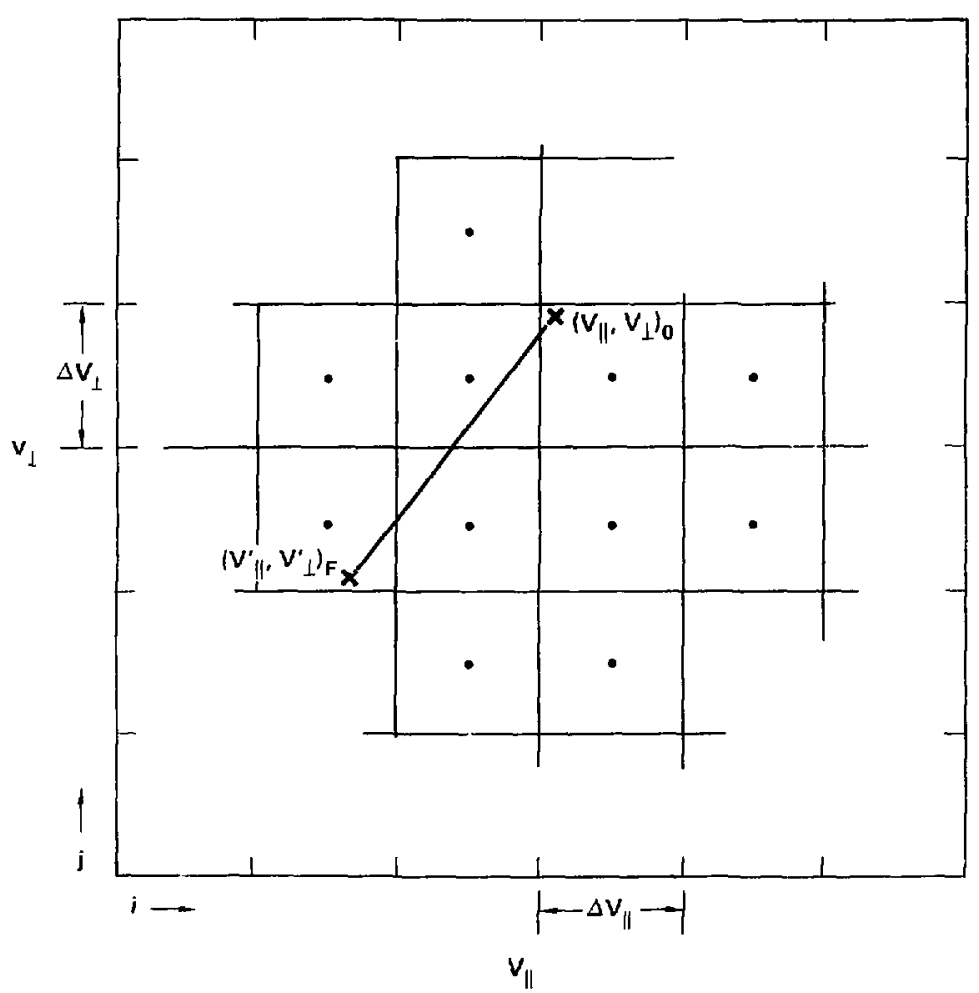

Figure 6.3 Model of alpha velocity space trajectory thriugh one collision sampling 
's not enough information at any one point to generate a distribution function for that point. In uniform plasmas, the radial position does not affect the velocity distribution, and an accurate distribution can be generated using information from collision sampling events occurring at different radial locations. Unfortunately, reactor plasmas are nonuniform and, in order to compensate for this problem, the plasma is divided into several radial zones. The plasma in each radial zone is assumed to be uniform, and a distribution function is generated for each zone. Tnis assumption is especially ralid for the innermost radial zones where temperature and density profiles are constant. Before presenting the full velocity distribution function, it is informative to ouserve a single particle as it slows in velocity space. The simulated alpha velocity space trajectories are not straight, as shown in Figs. 6.4 and 6.5 . Tlie particle in Fig. 6.4 was born with a $90^{\circ}$ pitch angle, while the particle in Fig. 6.5 was born on the loss cone with a $13^{\circ} \mathrm{pitch}$ angle. In both cases, the loss corie $1 \mathrm{~s}$ neglected and the random motion is due to angle scatter and dispersion. We can compare the calculated ratios of $\frac{\tau_{1}}{\tau_{D}}=5.9$, and $\frac{\tau_{\|}}{\tau}=129.0$ where $\tau_{\|}$is the dispersion time, with analytic approximations of 6 and 140 , respectively, These ratios show that dispersion and scattering are negligible in the Monte-Carlo slmulation for the high-energy alpha. But when we compare typical velocity changes, given by 


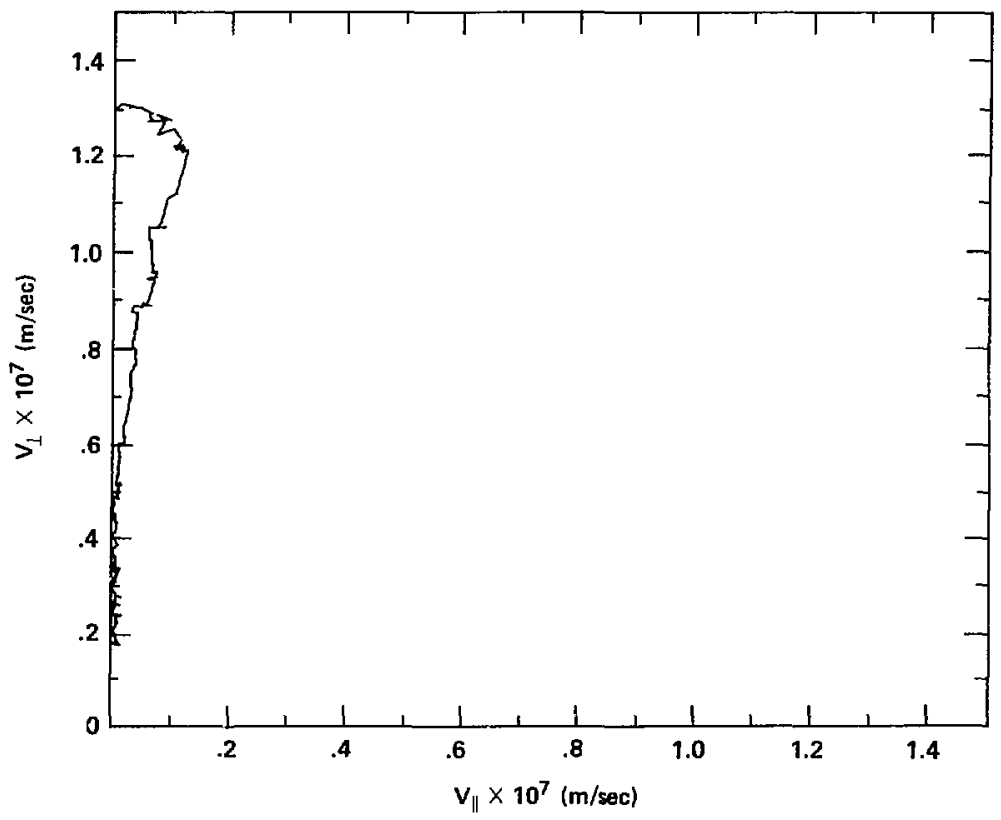

Figure 6.4 Velocity space trajectory from birth to thermalization for an alpha with an intial pitch angle of $90^{\circ}$. Loss cone angle 13 set to $0^{\circ}$ 


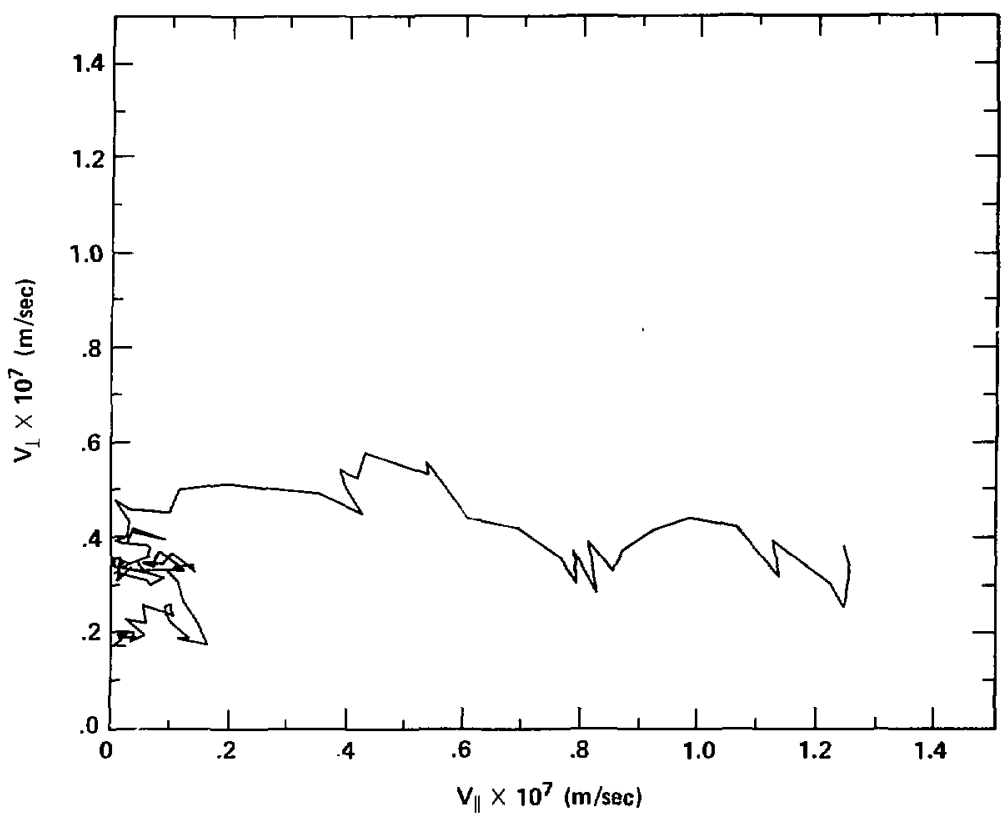

Figure 6.5 Velocity space trajectory from birth to thermalization for an alpha with an Initial pitch angle of , $13^{\circ}$. Loss cone angle is set to $0^{\circ}$ 


$$
\begin{aligned}
& \Delta w_{\| D}=\left\langle\Delta w_{\|}\right\rangle \Delta t=1.7 \times 10^{4} \mathrm{~m} / \sec \\
& \Delta w_{\|}=\left(3\left\langle\left(\Delta w_{\|}\right)^{2}\right\rangle \Delta t\right)^{1 / 2} R_{1}=7.1 \times 10^{4} \mathrm{~m} / \sec R_{1} \quad(6.2 .2 . \\
& \Delta w_{\perp}=\left(1.5\left\langle\left(\Delta w_{1}\right)^{2}\right\rangle \Delta t\right)^{1 / 2} R_{2}, R_{3}=2.3 \times 10^{5} \mathrm{~m} / \sec R_{2}, R_{3}
\end{aligned}
$$

for a collision sampling time $(\Delta t)$ of $\mathbf{i} \times 10^{-3}$ sec and a $3.52 \mathrm{MeV}$ alpha, it is seen that the dispersion and angle scatter changes depend on the random numbers $R_{1}, R_{2}$, and $R_{3}$, When a random number is large, i.e. approximately \pm 1 , then the scatter or dispersion can be equal to the drag; this causes the scatter in Fig. 6.4 and 6.5 . However, since $R_{1}, R_{2}$, and $R_{3}$ vary from -1 to +1 , their average value 1 s 0 ; therefore, the drag, in an average sense, dominates the slowing down process. Because of this average quality, we expect the particle trajectories, shown in Figs. 6.4 and 6.5 , to show general movement towar-d lower velocities, but they wlll not follow a radial line folning their velocity space birth position to the orlgin.

Individual particle trajectories, such as those shown in Figs. 6.4 and 6.5 , are summed to give a velocity distribution, shown in Figs. 6.6, 6.7, and 6.8. These distributions were made for the generic reactor parameters, and the distributions correspond to three radial zones across the plasma. The loss cone calculations have been included in these distributions, and the loss cone boundary is shown as the dashed line in the flgures. Note that the loss cone varies from one radial zone to the next because the plugging electrostatlc potential is proportional to $T_{1}$, which varjes with radius. As the potential 


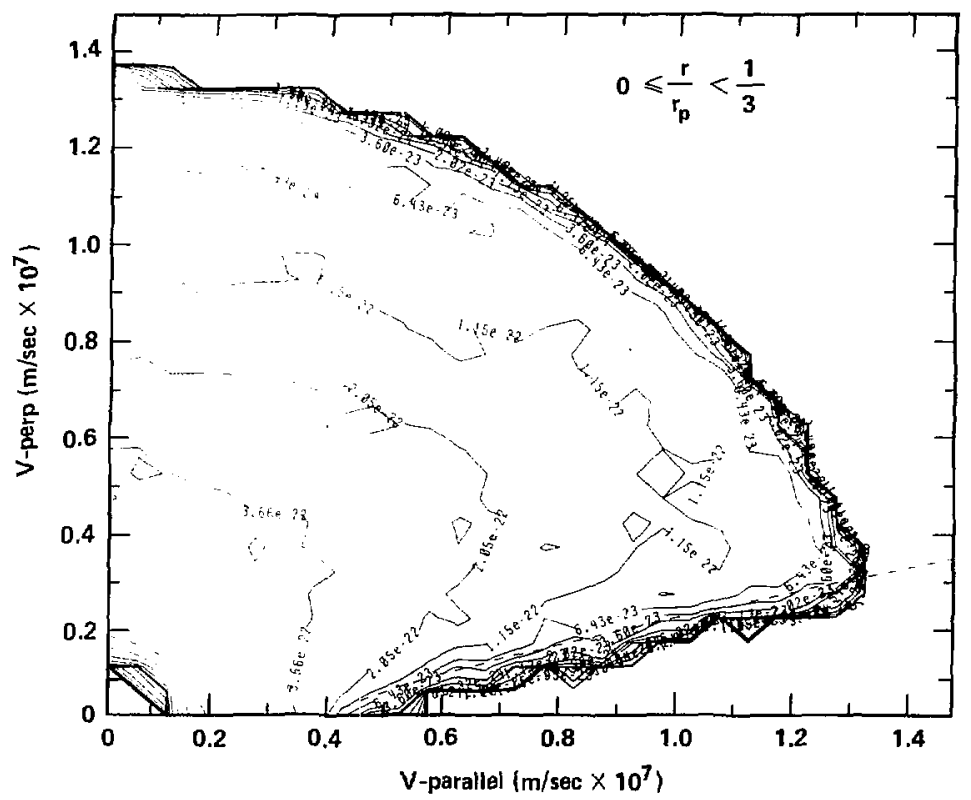

Figure 6.6 Alpha particle normalized distribution function for the generic reactor design. The distribution was produced by 1000 alphas followed from $3.52 \mathrm{MeV}$ to $60 \mathrm{keV}$ for $0 \leqslant r, r_{p}<1 / 3$ 


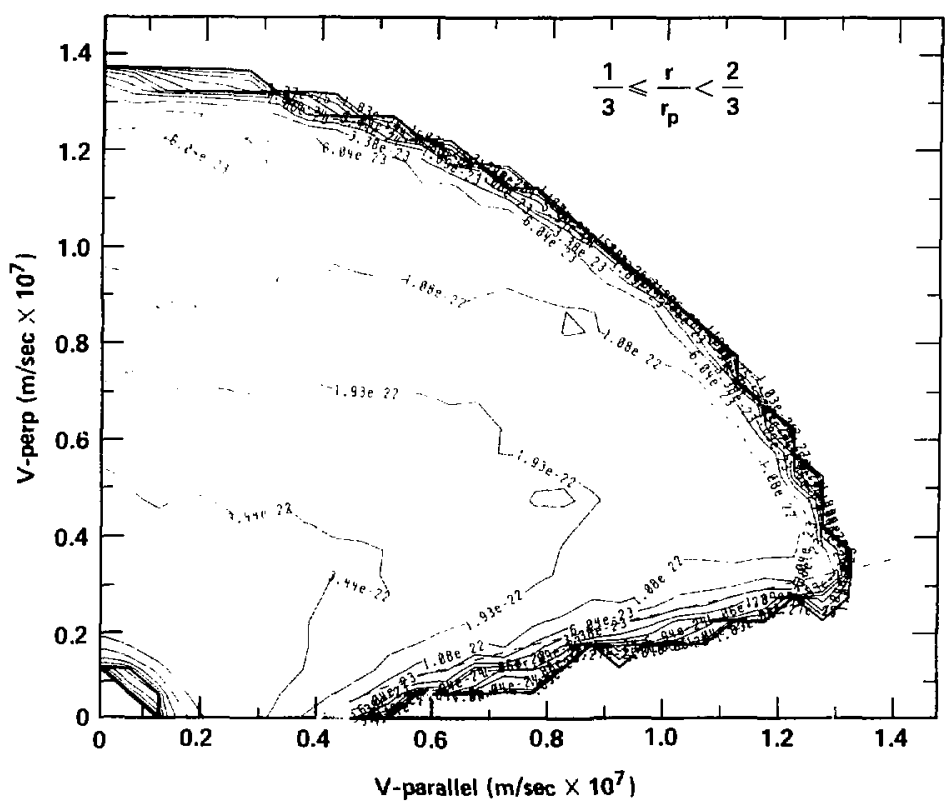

Figure 6.7 Alpha particle normalized distribution function for the generic reactor design. The distribution was produced by 1000 alphas followed from $3.52 \mathrm{MeV}$ to $60 \mathrm{keV}$ for $1 / 3 \leq r / r_{p}<2 / 3$ 


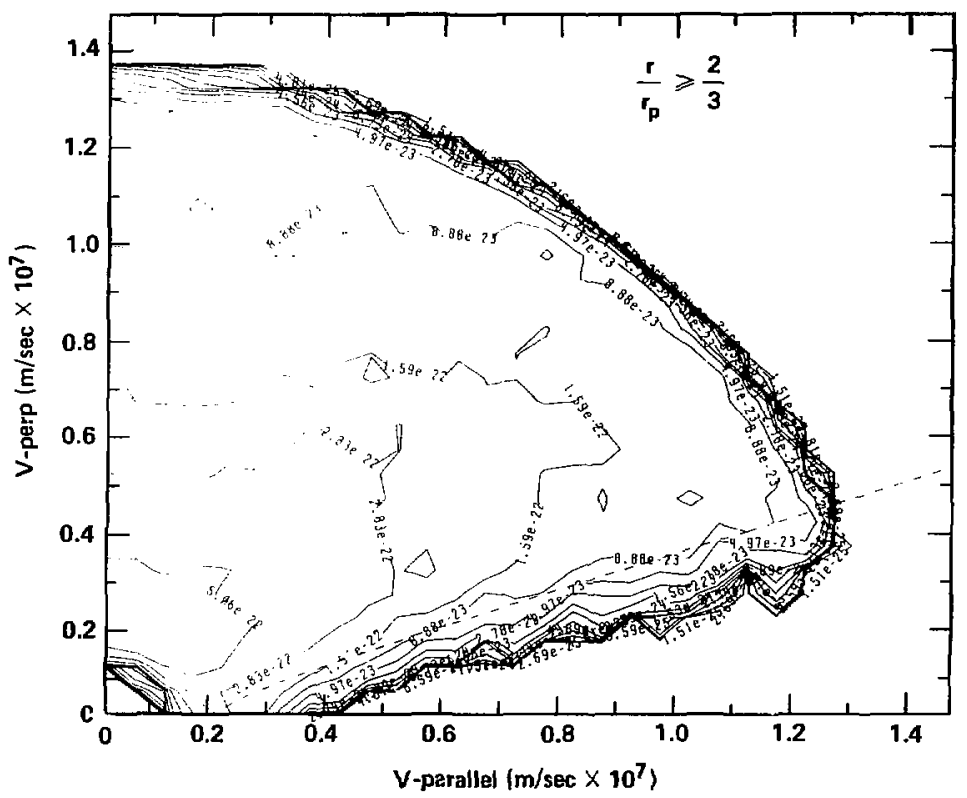

Figure 6.8 Alpha particle normallzed distribution function for the generic reactor design. The distribution was produced by 1000 alphas followed from $3.52 \mathrm{MeV}$ to $60 \mathrm{keV}$ for $2 / 3 \leq r / r_{p}<r_{w} / r_{p}$ 
decreases near the plasma edge, beta also goes to zero and the loss cone increases. The overlap of the distribution into the loss cone is caused by three factors. First, the loss cone boundary is determined for an average radial position inslde each radial zone. Because the plasma is nonuniform, the velocity distribution in each radial zone is an approximation to the distribution function at the average radial position and, therefore, there will be some coniribution to the distribution function that lies outside the calculated loss boundary. Second, the velocity bins desoribed in $\mathrm{Flg} .6 .3$ do not colncide with the loss cone boundary. This, coupled with a general conformal plotting routine, places some contours in the loss cone region. Finally, the method of calculation first samples a collision event, then determines the contribution of this event to the distribution function, and then checks to see if the particle is in the loss cone. This method will contribste information to the distribution function for that part of the particle trajectory in the loss cone. All of these problems can be corrected at the expense of a significant amount of additional computer time, but have not been Incorporated in this calculation,

Although the distribution functions shown in Figs. 6.6 through 6.8 are only approximations, they are a useful dlagnostic. Flrst, they show that there is a plateau in the distribution at velocities corresponding to $3.52 \mathrm{MeV}$. This plateau rapidly drops to zero for higher velocities. There is also a peak in the distribution at thermal energies where the alpha ash accumulates. The contour lines suggest 
that this peak gradually decreases to the high veloclty plateau. Finally, the circular contour lines above the loss cone boundary suggest that drag is the dominant energy loss process.

\subsubsection{Energy Distribution Function}

The energy distribution, defined as $f(\bar{r}, \varepsilon)$, defines the number of partfcles per unit volume, per unit energy, at some position in phase space. This distribution is calculated in the Monte-Carlo code by defining the distribution function in spherical coordinates as

$$
n(\bar{r})=\int_{\bar{v}} f(\bar{r}, v, \psi, \phi) v^{2} d v \sin \psi d \Psi d \Phi
$$

The integration over $\psi$ and $\phi$ is performed and the speed is transformed into energy coordinates to give

$$
\operatorname{dn}(\bar{r})=f(\bar{r}, \varepsilon) \pi\left(\frac{2}{m}\right)^{3 / 2} \varepsilon^{1 / 2} d \varepsilon
$$

The energy distribution is then defined as

$$
f(\bar{r}, \varepsilon)=\frac{\Delta n\left(r_{i}\right)}{\pi\left(\frac{2}{m}\right)^{3 / 2} \varepsilon_{1}^{1 / 2} \Delta \varepsilon^{*}}
$$

where Eq. (6.2.3.2) has been dlscretlzed Into small changes in density corresponding to respectlve energy changes. The alpha energy is 
divided into radial zones $\Delta \varepsilon^{*}$ wide with a mean energy of $\varepsilon_{i}$ in each zone. The density of particles $\Delta n\left(r_{1}\right)$ in each energy zone is proportional to the time the alpha spends in each zone. A Iinear interpolation technique, similar to the one used for the velocity distribution, is used to determine the time the alpha spends in each zone as it slows down during one collislonal sampling time. The total accumulated time in each energy bin 19 proportional to $\Delta n\left(r_{1}\right)$. The normalized distribucion function is then found from

$$
\hat{f}(\bar{r}, \varepsilon)=\frac{f(\bar{r}, E)}{N(r)}
$$

where $N(\bar{r})$ is proportional to $\Sigma_{i} \Delta t_{1}$ and the sum 19 the total thermalization time for all the particles making up the distribution. In terms of Eq. (6.8 and substituting $\Delta t_{i}$ for $\Delta n\left(\bar{r}_{i}\right)$, Eq. $(6.2 .3 .4)$ becomes

$$
\hat{f}(\bar{r}, \epsilon)=\frac{1}{\pi\left(\frac{2}{m^{\varepsilon} \varepsilon_{\alpha}}\right)^{3 / 2}\left(\frac{\varepsilon_{i}}{E_{\alpha}}\right)^{1 / 2} \frac{\Delta E^{*}}{\varepsilon_{\alpha}}} \frac{\Delta t_{i}}{\Delta T^{\prime}}
$$

where $\Delta T=I_{i} \Delta t_{1}$. A plot of $\pi\left(\frac{2}{m} \varepsilon_{\alpha}\right)^{3 / 2} \hat{f}(\bar{r}, \varepsilon)$ versus $\frac{\varepsilon_{1}}{\varepsilon_{\alpha}}$ for three radial zones of the plasma is shown in Figs. 6.9 through 6.11, where the Monte-Carlo calculated points are shown as crosses and the solid curve is a theoretical result. 


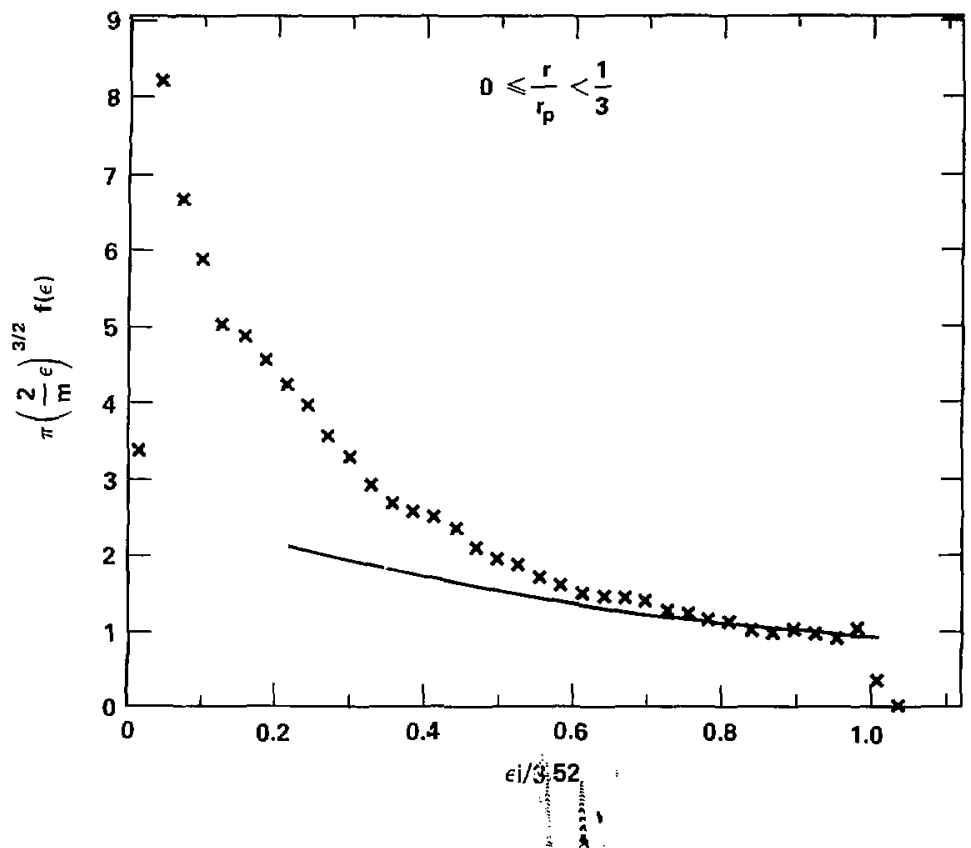

Figure 6.9 Energy distribution fund ich for the generic reaction design for 1000 alphas fillowed from $3.52 \mathrm{MeV}$ to $60 \mathrm{keV}$ for $0 \leq \mathrm{r} / \mathrm{r}_{\mathrm{p}}<1 / \mathrm{s}$ ( Solfd line is a theoretical approximatiof 16 


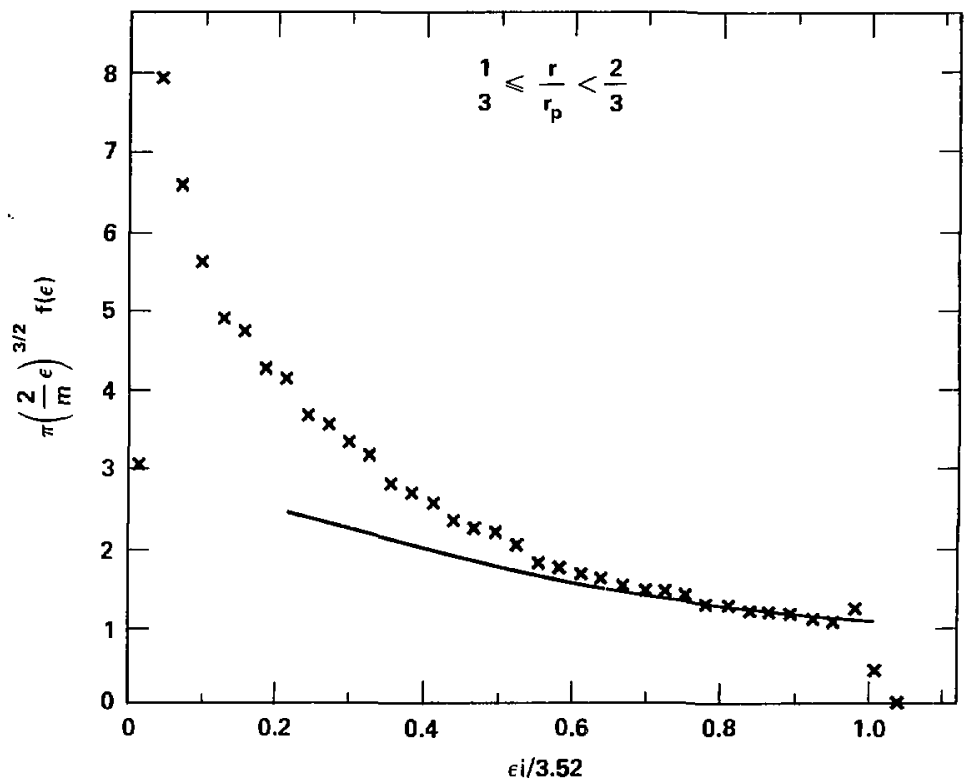

Figure 6.10 Energy distribution function for the generic reactor design for 1000 alphas followed from $3.52 \mathrm{MeV}$ to $60 \mathrm{keV}$ for $1 / 3 \leqslant \mathrm{r} / \mathrm{r}_{\mathrm{p}}<2 / 3(\mathrm{x})$. Solld line is a theoretical approximation ${ }^{16}$ 


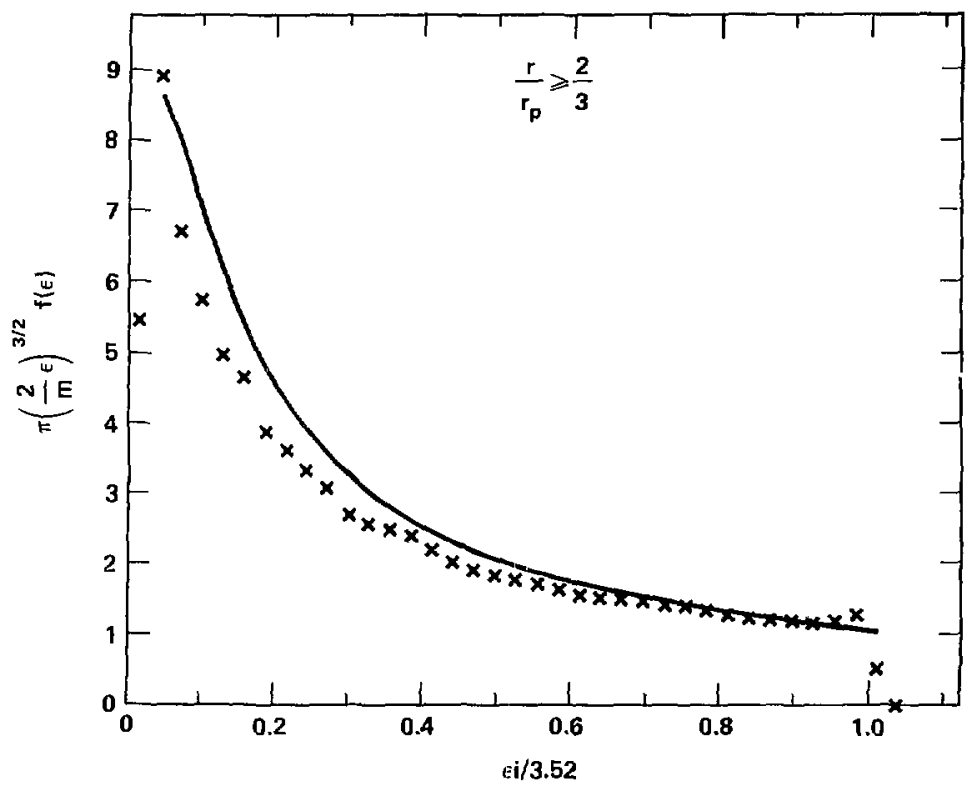

Ffgure 6.11 Energy distribution function for the generic reactor design for 1000 alphas followed from $3.52 \mathrm{MeV}$ to $60 \mathrm{keV}$ for $2 / 3 \leq \mathrm{r} / \mathrm{r}_{\mathrm{p}} \leq \mathrm{r}_{\mathrm{w}} / \mathrm{r}_{\mathrm{p}}(\mathrm{x})$. Solld line is a theoretical approximation 16 
A theoretical velocity distribution function for high energy neutral particles injected into a plasma has been derived by Cordey and Houghton. 47 This derivation was done assuming that injected particles are dragBed down to thermal energies in a uniform plasma by ions and electrons. Large angle scattering events are neglected and the derivation is valid in the region where $v>v_{c}$, where $v_{c}$ is the velocity at which the energy transfer from hot ions to cold electrons and to cold ions is equal. The source function used in this derlvation is typical of neutral injection systems. Driemeyer ${ }^{16}$ has transformed this distribution into an energy distribution function and incorporated an alpha particle source term. This normalized distribution function is given by

$$
\hat{f}(\bar{r}, \varepsilon)=\frac{C o}{N(r) E_{\alpha}} \frac{\left(\frac{E}{E_{\alpha}}\right)^{1 / 2}}{\left(\frac{\varepsilon}{\varepsilon_{\alpha}}\right)^{3 / 2+}\left(\frac{\varepsilon_{c}}{\varepsilon_{\alpha}}\right)^{3 / 2}}
$$

where $\frac{\varepsilon_{c}}{\varepsilon_{\alpha}}$ is $\left(\frac{3 \pi^{1 / 2}}{4} \frac{m_{e}}{m_{1}}\right)^{2 / 3} \frac{m_{\alpha}}{m_{e}} \frac{T_{e}}{\varepsilon_{\alpha}}$ and $\frac{\text { Co }}{N(r) \varepsilon_{\alpha}}$ is the normalization constant. The theoretical and Monte-Carlo energy distributions given by $E q .(6.2 .3 .5)$ and $(6.2 .3 .6)$ are equal at energies near $3.52 \mathrm{MeV}$, and therefore the normalization constant is found from equating Eq. $(6.2 \cdot 3.5)$ to $(6.2 .3 .6)$ and is given $r y$

$$
\frac{C_{0}}{N(\bar{r}) \Sigma_{\alpha}}=\frac{1}{\pi\left(\frac{2}{m} \varepsilon_{\alpha}\right)^{3 / 2}} \frac{\left(\varepsilon_{\alpha} / \varepsilon_{\alpha}\right)^{3 / 2}+\left(\varepsilon_{\alpha / \alpha}\right)^{3 / 2}}{\left(\varepsilon_{\alpha} / \varepsilon_{\alpha}\right)\left(\Delta \varepsilon^{* / \varepsilon_{\alpha}}\right)} \frac{\Delta t^{*}}{\Delta T}
$$


Where $\Delta t_{1}^{*}$ is proportional to the alpha density in the energy bin, where the constant is evaluated. To reduce statistical variations, we averaged the normalization constant over ten Monte-Carlo energy bins near $3.52 \mathrm{MeV}$.

A comparison between the energy distribution generated by the Monte-Carlo simulation and the theoretical distribution is shown in Fig. 6.9 through 6.11. From these plots, we see that the Monte-Carlo generated distribution has the same slope at high alpha energles, but varies from theory as the alpha thermalizes. The difference between the Monte-Carlo calculation and theory occurs for several reasons. First, the theory is valld for the case where $v_{i}<\ll v_{\alpha} \ll<v_{e}$. For alpha particles in a plasma with ion temperatures of $40 \mathrm{keV}$ and electron temperatures of $28 \mathrm{keV}$, the $\operatorname{rat10} \frac{v_{i}}{v_{\alpha}}=0.14$ and $\frac{v_{e}}{v_{\alpha}}=7.6$. These ratios do not explicitiy satisfy the velocity condition given above. Another difference oc surs because the Monte-Carlo calculation includes velocity dispersion and angle scatter. Although these processes become dominant for alpha energins less than $500 \mathrm{keV}$, they do affect alpha thermalization at hlgher energies. This is evident from the fact that velocity space collisionally lost alphas leave with an average energy of approximately 1.5 MeV (see Table 6.1). Finally, a simple drag model for the plasma shows that the energy distribution function is proportional to $\mathrm{E}^{-1}$, whereas the theoretical distribution function $1 \mathrm{~s}$ proportional to $\varepsilon^{-1}\left[1+\left(\frac{\varepsilon_{c}}{\varepsilon_{\alpha}}\right)^{3 / 2}\right]^{-1}$. The effect of $\varepsilon_{c}$ on the distribution function is shown in F1g. 6.12, where the alphas are 
TABLE 6.1.

STATISTICAL ANALYSIS OF THE RESULTS FOR THE GENERIC KEACTOR DESIGN

\section{Description}

$\mathrm{N}_{\mathrm{c}} / \mathrm{N}_{\mathrm{T}} \quad 0 \leq \frac{r}{r_{\mathrm{p}}} \leq 0.864$

$\mathrm{N}_{\mathrm{h}} / \mathrm{N}_{\mathrm{T}} \quad \frac{\mathrm{r}}{\mathrm{r}_{\mathrm{p}}}>0.864$

$\mathrm{N}_{\mathrm{L}} / \mathrm{N}_{\mathrm{T}}$ Lost immeriately

$\mathrm{N}_{\mathrm{CL}} / \mathrm{N}_{\mathrm{T}}$ Collisionally lost

$\varepsilon_{\mathrm{c}^{\prime}} \varepsilon_{\mathrm{T}} \quad 0 \leq \frac{\mathrm{r}}{\mathrm{r}_{\mathrm{p}}} \leq 0.864$

$\varepsilon_{h} / \varepsilon_{T} \circ \frac{r}{r_{p}}>0.864$

$\varepsilon_{\mathrm{L}} / \varepsilon_{\mathrm{T}} \quad$ Lost immediately

${ }^{E_{C L}}{ }^{\prime \varepsilon_{T}}$ Collisionally lost

$\varepsilon_{t h} / E_{\tau}$ Thermal energy

〈B⿱ $\rangle$

$\langle\beta\rangle$

$\mathrm{B}_{\mathrm{v}}$

$r_{p}$

$r_{c}$

$p / r_{e}$

(ii) Collistonally lost a's (MeV)

$\Delta \varepsilon_{i} / \varepsilon_{\alpha}$

0.21

0.79

0.51

0.03

0.8

0.4

3.0

0.5

1.4
7000-Particle Run Standard

Deviation

Mean Deviation

0.01

0.51

0.35

0.01

0.35

0.03

0.01

0.03

0.10

0.01

0.11

0.58

0.01

0.58

0.32

0.01

0.33

0.01

0.03

0.04

0.05

0.02

0.02

0.8

0.4

3.0

0.5

0.43

0.43

0.27

0.27

1.6

$\Delta \varepsilon e^{\prime \varepsilon}{ }_{\alpha}$
0.21

0.79 


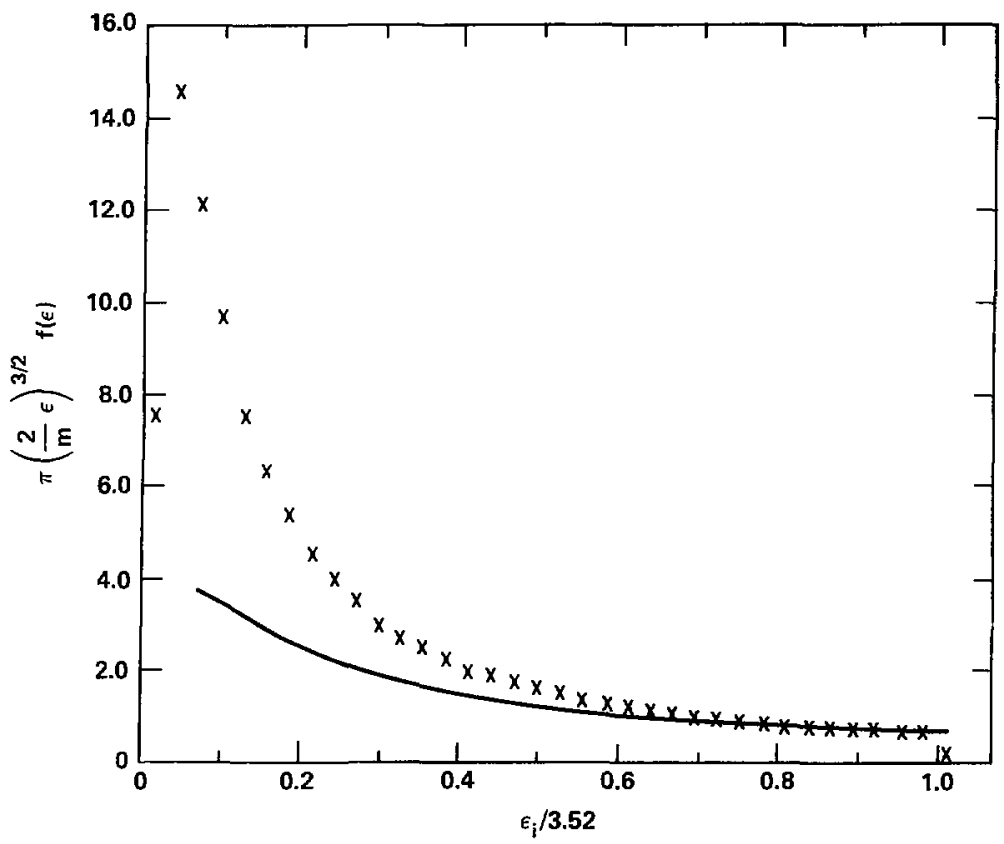

Eigure 6.12 Energy distribution function predicted by Monte-Carlo teahniques $(x)$ and by Drlemeyer ${ }^{16}$ (-) for an alpha in a uniform plasma at $10 \mathrm{keV}$ 
slowing down in a uniform plasma at $10 \mathrm{kev}$. Th1s $1 \mathrm{~s}$ similar to F1g. 6.11 where the alphas are near the edge of the plasma and the plasma temperature used in determining $\varepsilon_{c}$ is $7 \mathrm{keV}$.

\subsubsection{Guiding Center Drift Plotg}

This final diagnostic plots the absolute value of the guiding center coordinates at the plasma midplane after a precietermined number of collision sampling times have occurred. These plots have been Incorporated as a visual picture of the guiding center movement under the influence of the gradient drag drift. Figures 6.13 through 6.15 show the guiding center radial movement in the generic reactor design after .25 , and 50 collision sampling times. Thermal particle positions are not shown on these plots, nor are the particles that are lost out the loss cone between plots. The important fact that these plots show is that the radial guiding center drift can be large and that alpha particles drift out to the plasma edge in less than 25 sampling times, which is on the order of milliseconds for this model. Therefore, for the generic reactor model a signiflcant amount of alpha eriergy and ash accumulates at the edge of the plasma. other temperature and density models can change this drift and will be considered in Section 6.4.

\subsection{Equal Density and Temperature Scale Length Results}

The reactor model that was first studied required the plasma density and temperature profiles to reach their halo values at the $r_{p}$ ' the edge of the plasma. The plasma radius thesefore defined the 


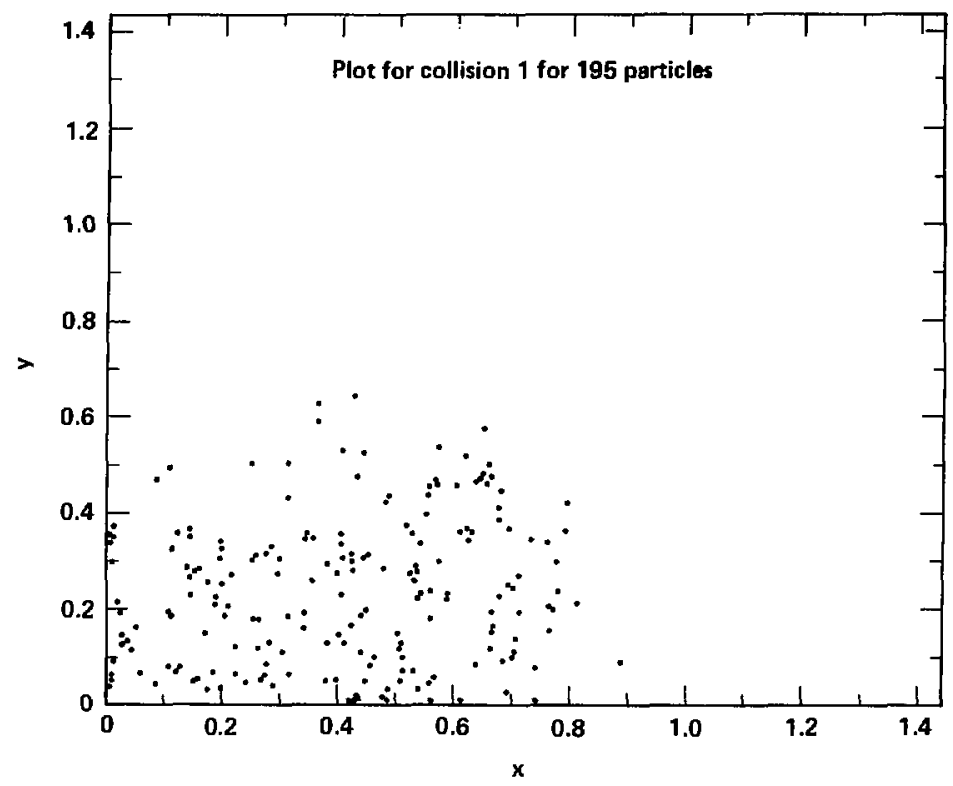

Figure 6.13 Alpha guiding center position after one collision sampling time for the generic reactor design 


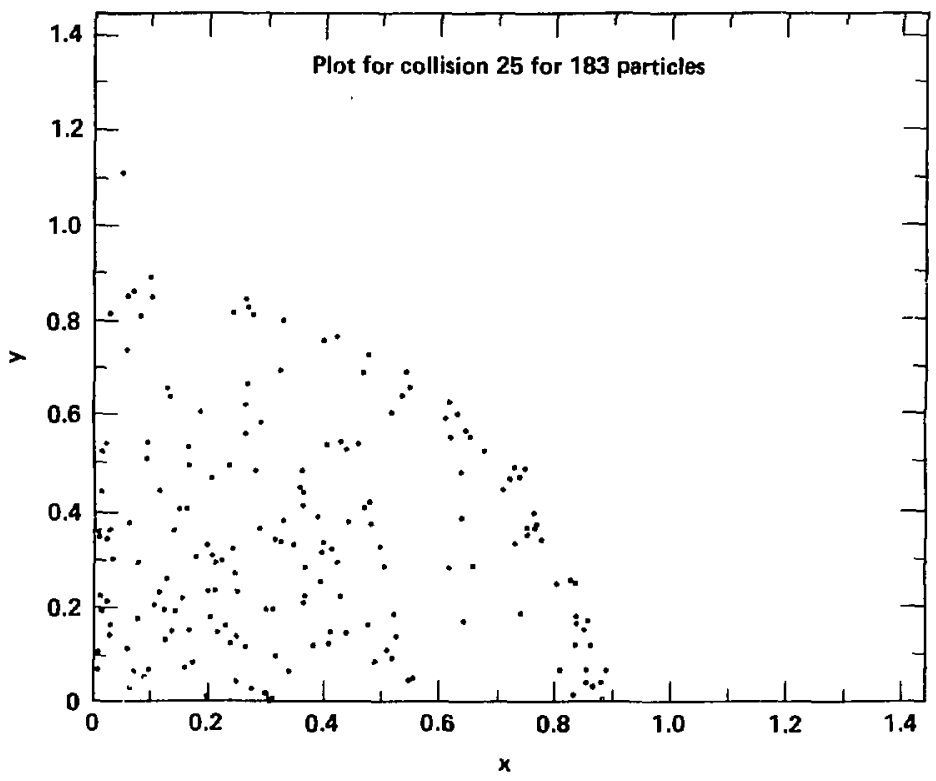

Figure 6.14 Alpha guiding center position after 25 collisions sampling times for the generic reactor design 


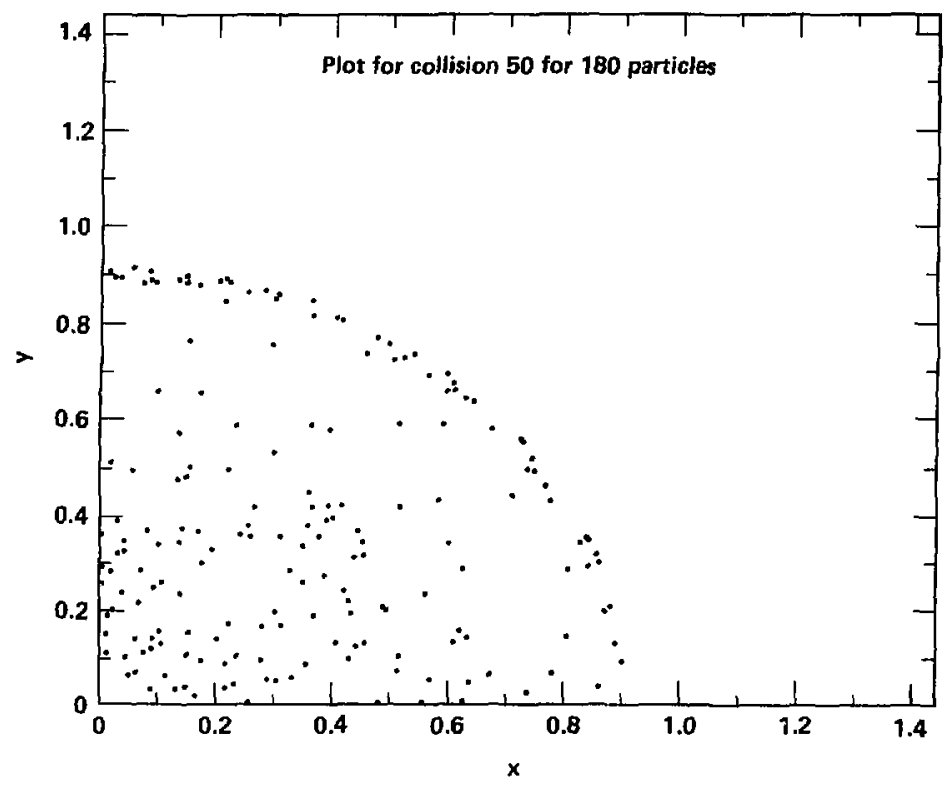

Figure 6.15 Alpha guiding center positior after 50 collisions sampling times for the generic reactor design 
density and temperature scale length. This single-scale-length problem attempted to model reactor studles that place a sharp boundary between the plasma core and halo, with the core plasma belng defined for $\frac{r}{r_{p}}<1$. In this study, the sharp boundary is replaced by a smooth transition which requires the temperature and density to decrease in the core plasma. When the temperature and density decrease in the core, the alpha reaction rate $\left(\dot{N}_{\alpha}\right)$ becomes negliglble inside the plasma near $\frac{r}{r_{p}}=1$.

\subsubsection{Generic Reactor Des1 gn}

Parameters for a generic reactor have been given in Table 1.1 and the density and temperature profiles are shown in Fig. 5.2. The alpha particle birth distribution was shown in Fig. 4.2 and 4.3 . The radial guiding center drift causes particles to accumulate near the plasma edge, as shown in Fig. 6.13 through 6.15. The detailed MonteCarlo results for this generic design are described in the following sections.

\subsubsection{Alpha Partiole Thermalization}

The Monte-Carlo calculation follows particles from birth to thermalization and stores the thermal alpha location in a radial bin. As the number of alpha particles increases, the number in each bin increases and the radial distribution attains some distinct profile, By normalizing this distribution with the total number of particles that are started, a steady state thermalized distribution is found (Fig. 6.16). The ratio is zero at the origin since the bin size goes 


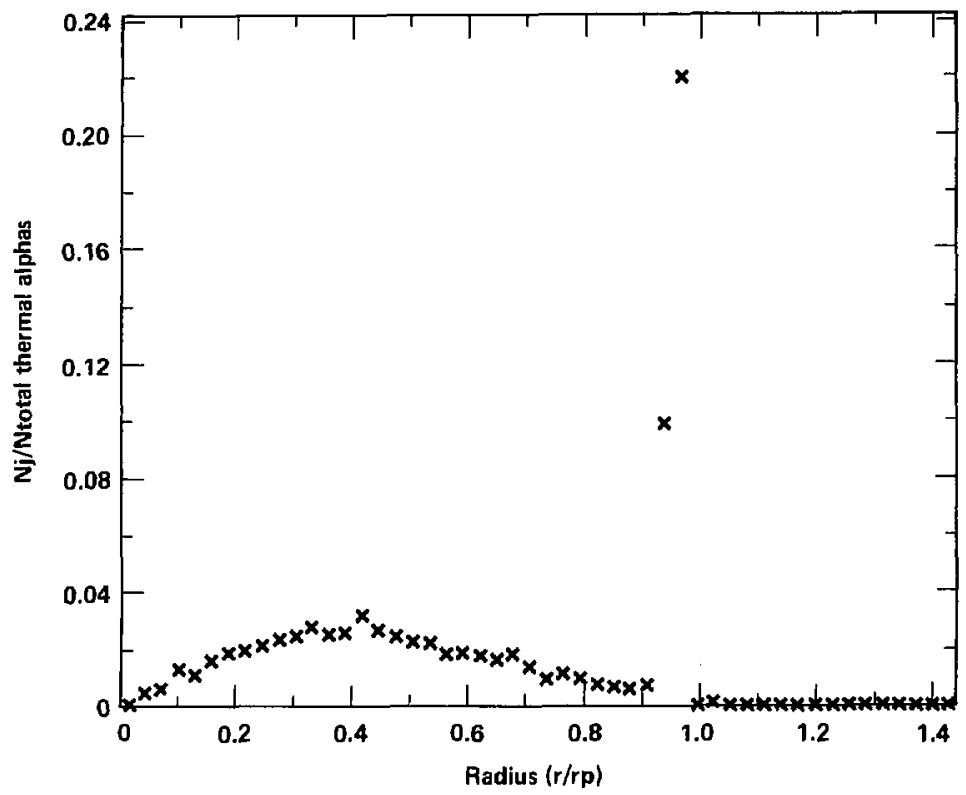

Figure 6.16 Thermal alpha particle radial profile normalized to the total number of alphas followed, for the generic reactor design 
to zero, and the ratio is zero at large values of $\frac{r}{r_{p}}$ since the $\hat{N}_{\alpha}$ goes to zero near $r_{p}$, and the alphas are limited in their radial diffusion. The alpha density in each bin is given by

$$
n_{j}=\frac{N_{j}}{\Delta V_{j}}
$$

where $\left.\Delta V_{j}=\pi\left(r_{j+1}^{2}-r_{j}^{2}\right)\right) L$ and $L$ is the plasma length. The density ratio $\frac{n_{j}}{n_{T}}$ can be computed and is plotted in FiB. 6.17 . The scatter near the origin is due to the $f$ act that small deviations in the caloulated distribution will cause large variations in the density since the bin volume is small. Note that the profile has a negative slope for $\frac{r}{r_{p}} \leq 0.7$, indicating an outward radial diffusion of the themal particles, which should follow classical diffusion rates. There is also a large density splke at the edge of the plasma caused by the radlal guiding center drift.

A particle balance on a radial bin can be used to find the thermallzation ratio in each bin. The balance equation can be stated as

$$
\left|\begin{array}{l}
\text { Rate at which } \\
\text { alphas thermalize } \\
\text { in bin } J
\end{array}\right|=\left|\begin{array}{l}
\text { Rate at which } \\
\text { particleg are } \\
\text { accumulated in } \mathrm{J}
\end{array}\right|-\left|\begin{array}{l}
\text { Rate at which } \\
\text { particles are } \\
\text { lost from } \mathrm{J}
\end{array}\right|
$$

Alphas are inltially born in bin $j$ at the rate of $n_{D J} n_{T j}\left\langle a v{ }_{j}\right.$, and their trajectories enable thern to sample many bins before they 


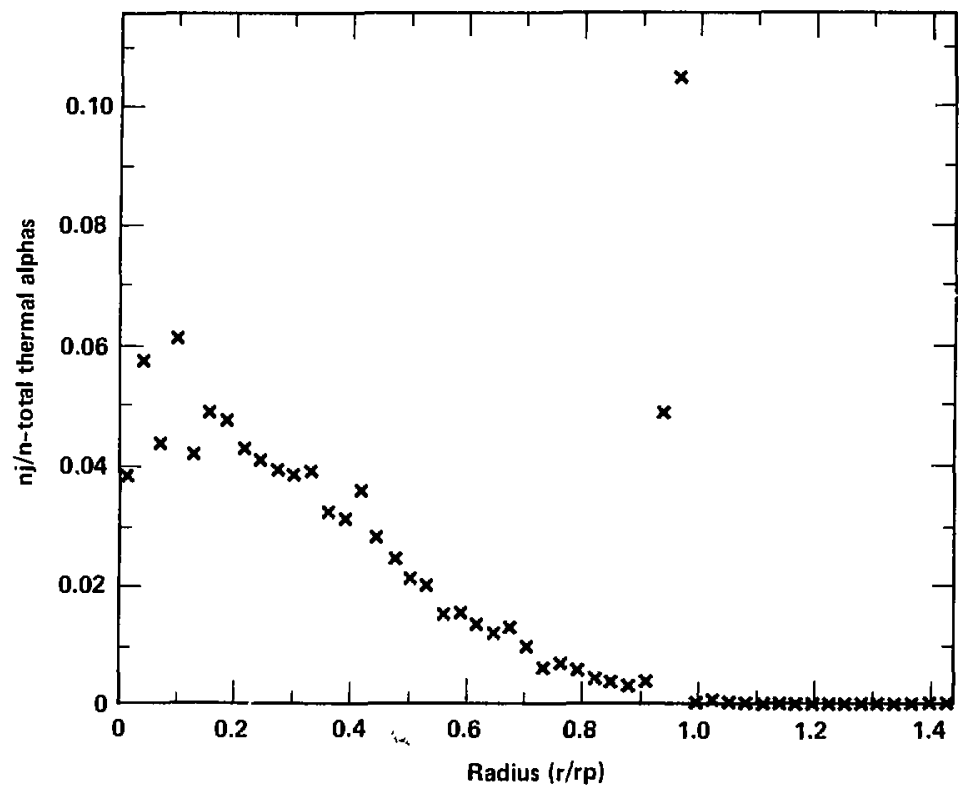

F1gure 6.17 Thermal alpha radial density profile normalized to the total number of alphas followed divided by the plasma volume, for the generic reactor design 
thermalize. After an alpha is born in bin $j$, it can leave this bin because of its trajectory or because of velocity space losses. The flux of alphas through any bin can be written as $\left\langle\bar{v}_{\alpha}\right\rangle \cdot \nabla n_{\alpha}$ where $\left\langle\bar{v}_{\alpha}\right\rangle$ is the alpha fluid speed. The velocity space losses occur when the alpha is elther born in the loss cone or when it is pitch angle scattered into the loss cone. The prompt or birth losses are given by $\frac{1}{2 R_{j}} n_{D j} n_{T j}\langle\sigma V\rangle_{j}$ where $R_{j}$ is the mirror ratio for bin $j$. By collecting these terms the thermalization rate in bin $j$ becomes

$$
\frac{\partial n_{\alpha j}}{\partial t}+\left\langle\bar{v}_{a}\right\rangle \cdot \nabla n_{\alpha j}=n_{D j} n_{T j}\langle\sigma v)_{j}\left(1-\frac{1}{2 R_{j}}\right)-C 1
$$

where $C l$ represents the collislonally lost partioles. By integrating this equation with respect to time, the thermalized alpha density in bin $j$ can be found and the total number lensity is the sum of the bin densities.

$$
n_{\alpha}=\sum_{j} n_{\alpha j}
$$

The Monte-Carlo calculation integrates Eq, (6.3.1.1.2) by following alpha trajectorles which produce the bin number densities, $n_{j}$. The thermalization rate for the $j^{\text {th }}$ bin must be ealoulated using the solution to the integrated equation. We do this by assuming that the thermalization rate is proportional to the total alpha birth rate and Is defined as 


$$
\frac{d n_{a j}}{d t}=\frac{n_{a J}}{n_{\alpha T}}\left(n_{D} n_{T}\langle\sigma v\rangle\right)_{T}
$$

where the subscript $T$ refers to an average value defined by $\frac{\int_{V} g(v) d v}{\int_{V} d v}$. A plot of the alpha thermalization rate as a function of radius is shown in Fig. 6.18 .

Finally, we observe that the thermalization rate for the generic design has a sharp spike near the edge of the plasma. The number of alphas that move from their birth position to the edge can be shown by plotting the alpha birth position mirus the thermal position (F1g. 6.19). The majority of thermal alphas near the plasma edge come from near the cencer of the plasma $\left(\frac{r}{r p}-0.6\right)$ where $B$ is still large and the average Larmor orbit extends into the halo. The alpha thermal minus birth position is essentially zero for $\frac{r}{r p}<0.2$, which implies that the particles born near the axis see a uniform plasma and experience little radial motion during the thermalization pr scess. This large spike in the thermal alpha density suggests that the plasma density ar. 1 temperature proflle models need changing so that a uniform thernal profile can be obtalned.

\subsubsection{Alpha Particle Guiding Center Thermalization}

Guir'ng center distributions and thermalization rates are determined in the same manner as the alpha density distributions. A comparison between the gulding center and particle density distributions for thermal alphas is shown in Fig. $6 \approx 0$. The alpha Larmor thermal radius is small compared to the characteristic sale 




Figure 6.18 Alpha particle thermalization rate for the generic reactor design 


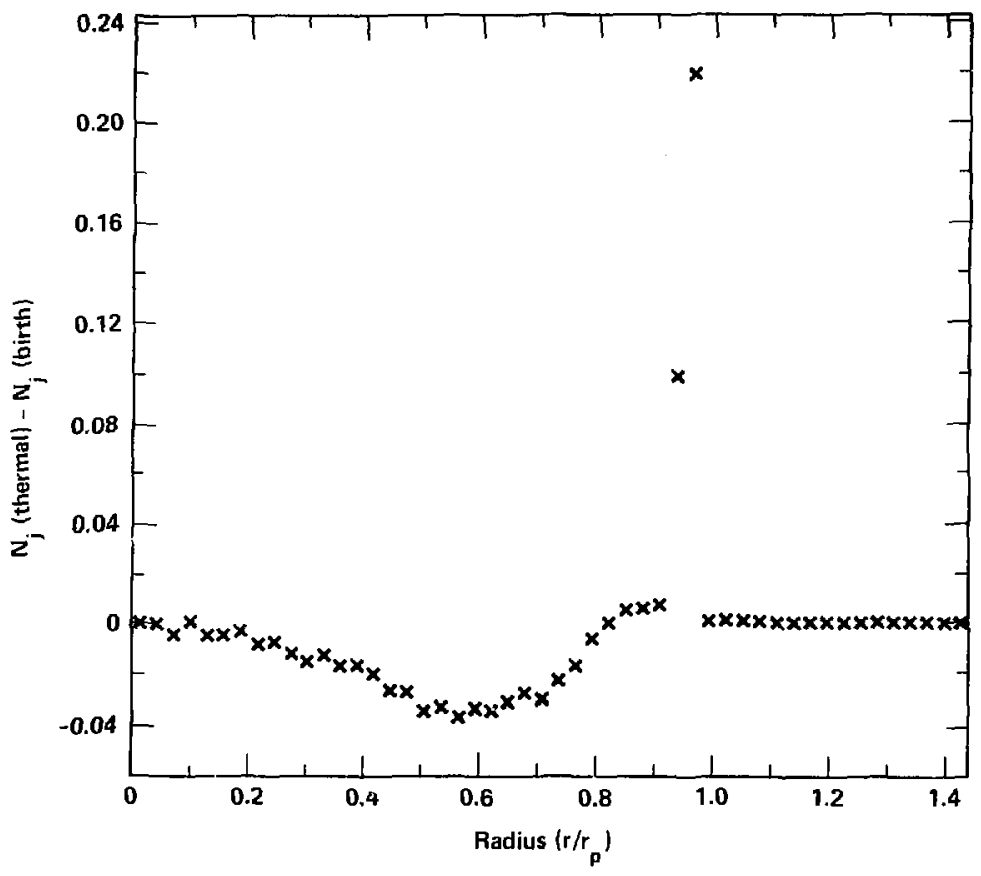

Figure 6.19 Number of alpha particzes born minus the number of alpha particles thermalizing in $r_{j}$ 


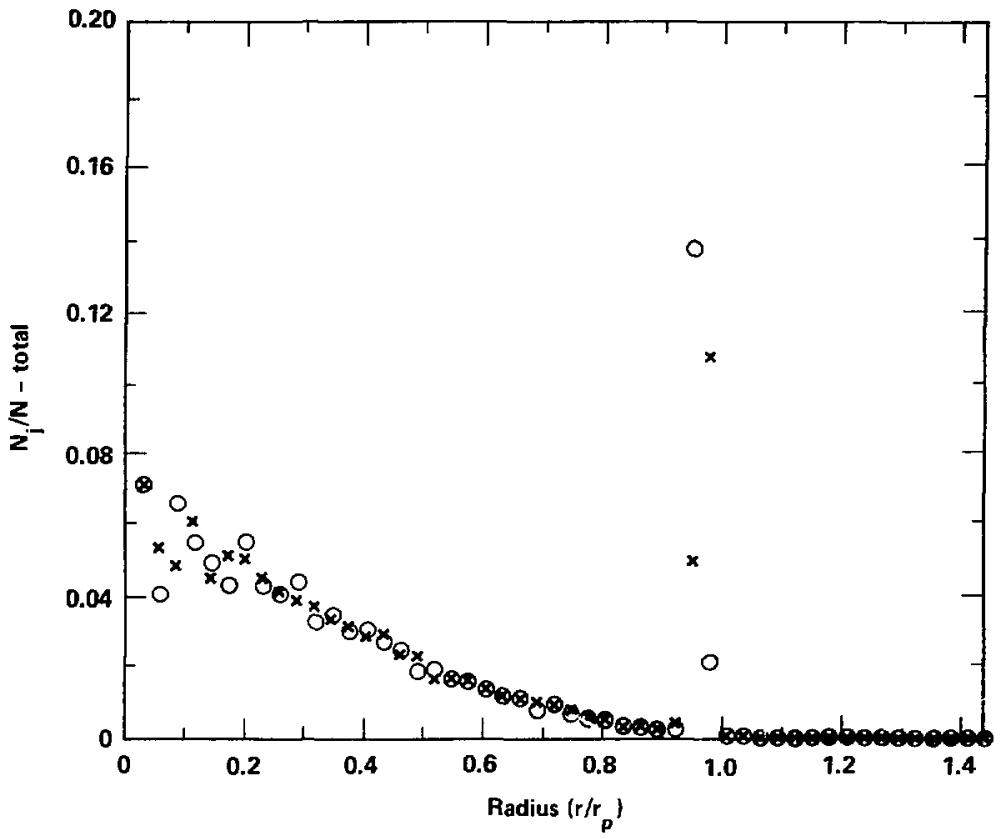

Figure 6.20 Alpha particle( $x$ ) and guiding center(0) normalized thermal densities as a function of radius 
length (L) in the reactor core $\left(\frac{r}{r p} \leq 0.864\right)$, but becomes comparable to the scale lengths at the plasma edge $\left(0.864<\frac{r}{r p} \leq 1\right)$.

In the region where $\frac{\rho}{L} \sim 1$, which occurs near the plasma edge, the relation between particle density and guiding center density $1 \mathrm{~s}$ given by 48

$$
n(r)=\frac{2}{\pi} \int_{r-\rho}^{r+\rho} \frac{N(R) R d R}{\left[4 R^{2} r^{2}-\left(R^{2}+r^{2}-\rho^{2}\right)^{2}\right]^{1 / 2}}
$$

where $N(R)$ is the guiding center density at $R$ and $r$ is the particle radius. This is a local relation whlch determines $n(r)$ over a Larmor diameter. If $N(R)$ equals a constant $N_{0^{\prime}}$ then $n(r)=N_{0^{\circ}}$ If $N(R)$ is quadratic, such that $N(R)=N_{0}\left(1-\frac{R^{2}}{r_{p}^{2}}\right)$, then the above integral becomes

$$
n(r)=N_{0}\left(1-\frac{r^{2}+\beta^{2}}{r_{p}^{2}}\right)
$$

which can be changed to

$$
n(r)=N(R)+N_{0}\left(\frac{R^{2}-\left(r^{2}+\rho^{2}\right)}{r_{p}^{2}}\right)
$$

Note that $n(r)$ is less than the $N(R)$ when $R^{2}>r^{2}+\rho^{2}$ and $n(r)$ is greater than $N(R)$ when $R^{2}>r^{2}+\rho^{2}$. A slmilar phenomenon occurs near the plasma edge in the generic design and can be seen in Fig. 6.20 for 
$0.9<\frac{r}{r_{p}}<1$. The difference between guiding center density and particle density when $\frac{\rho}{\mathrm{L}} \sim 1$ can also be seen by comparing the alpha birth densities (Fig. 6.21).

When $\frac{\rho}{L}$ is small, the relation between particle density and guiding center density can be appraximated by 49

$$
n(r)=N(r)+\frac{\rho}{4} \nabla^{2} N(r)+\ldots
$$

where $n(r)$ is the particle density, $N(r)$ is the guiding center density, and $\nabla^{2}$ is the Laplaciin perpendicular to $\bar{B}$. In Fig. 6.20, for $\frac{r}{r_{p}}<0.864$, the guiding center density is nearly linear, and $\nabla^{2} N(r)-0 ;$ therefore, $n(r)=N(r)$.

\subsubsection{Alpha Karticle Energy Distribution}

The amount of energy given to each radial bin is determined as the alpha slows down. The total energy deposited in each bin $\left(\varepsilon_{j}\right)$, divided by the total alpha energy avallable $\left(\varepsilon_{\alpha} \mathrm{N}_{T}\right)$, is shown in F1g. 6.22 for the generic reactor design. This energy ratio is similar to the thermal alpha particle ratio since $\frac{\varepsilon_{j}}{\varepsilon_{\alpha} N_{T}}$ goes to zero at $\frac{r}{r_{p}}=0$, and it is also zaro for $\frac{r+2 \rho}{r_{p}} \geq 0$. The radial distribution of $\frac{\varepsilon_{j}}{\varepsilon_{\alpha} N_{T}}$ for tho generlo design has a peak near the f? zsma edge, caused by the radial guiding cerier orlft. 


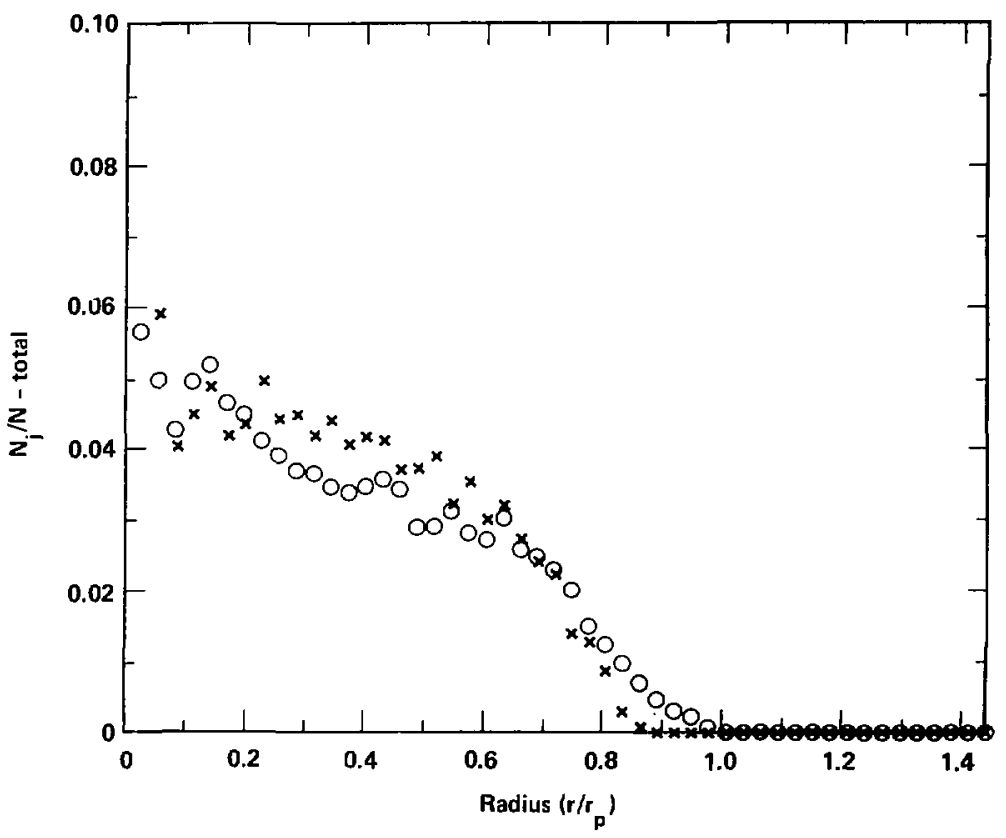

Figure 6.21 Alpha particle(x) and guiding center(o) normalized birth densities as a function of radius 


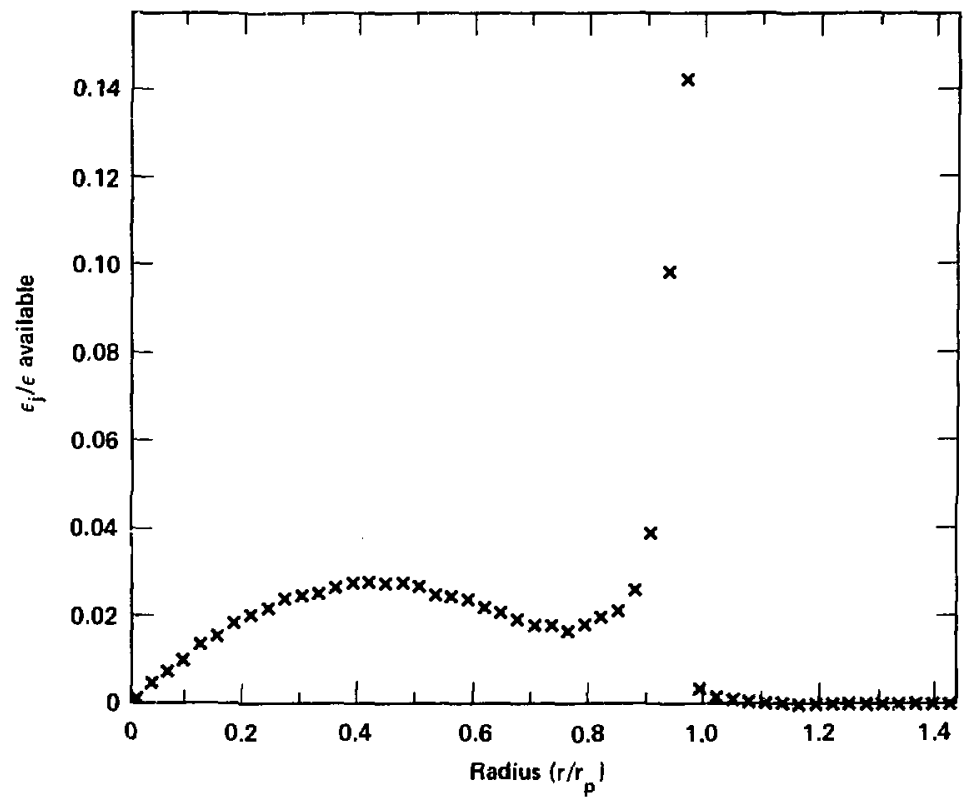

Figure 6.22 Normalized alpha energ; deposition profile for $t$ generic reactor design 
The energy transferred per unit volume of the plasma, 1.e., $e_{j}=\frac{\varepsilon_{j}}{\Delta V_{j}}$ can be computed, and the ratio of $\frac{e_{j}}{e_{T}}$, where $e_{T}=\frac{\varepsilon_{\alpha} N_{T}}{V}$, can be determined and is plotted in Fig. 6.23.

The ratio of $\frac{e_{j}}{e_{T}}$ can be used to determine the alpha power into each radial bin. This can be calculated in a similar mant ir as the particle thermalization rate, with the result given by

$$
P_{j}=\frac{e_{j}}{e_{T}} \varepsilon_{\alpha}\left(n_{D} n_{T}\langle\sigma v\rangle\right)_{T}
$$

and shown in Fig. 6.24. The alpha power to the lons and electrons is shown in Fig. 6.25, and the fraction of energy to each species is given in Table 6.1. The alpha power to the plasma along with the alpha energy fraction to the plasma fons and electrons 13 necessary for designing future fusion reactors.

\subsubsection{Stat1st1cal Analys 1s}

The data and figures that have been presented are results from the generic reactor design. These $\mathrm{r}$ esults were generated using 7000 alphas and checked by using nine separate calculations consisting of 1000 particles per run, in which the seed given to the random number generator was changed in each run. In analyzing these results, we have divided the plasma into two regions. The first region is the not reacting plasma core, $0 \leq \frac{r}{r_{p}} \leq 0.864$, which corresponds to the radial length over which the alphas are born. The second region, $0.864<\frac{r}{r_{p}}$, 


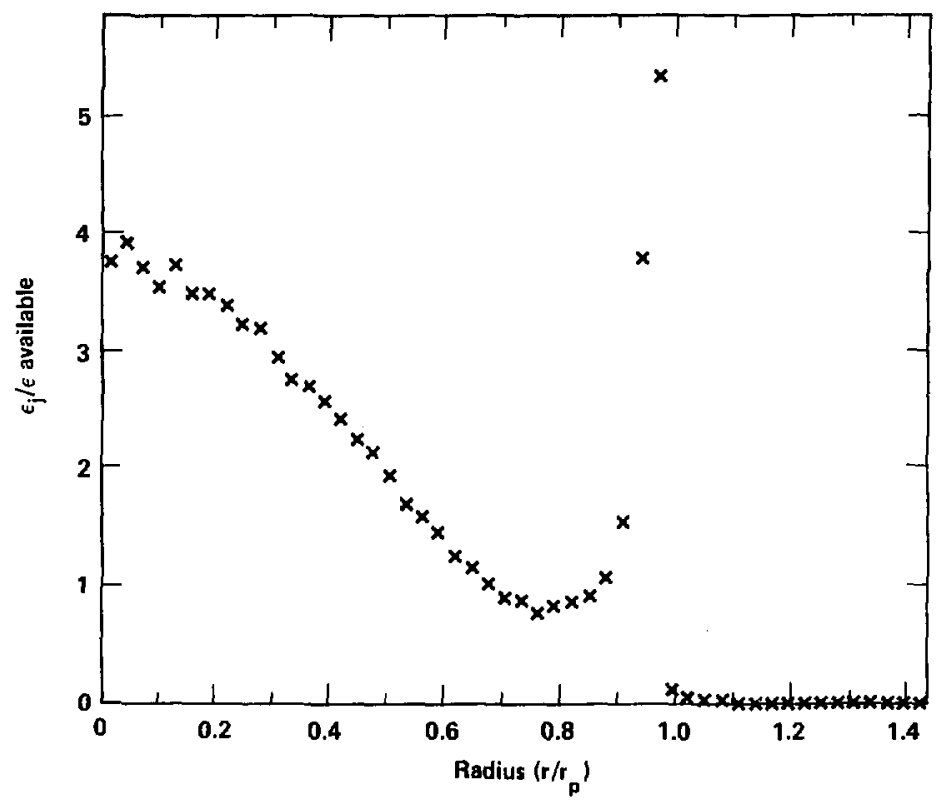

Figure 6.23 Alpha energy deposition profile per unit volume normallzed to the total energy deposited in the total plasma volume 




Figure 6.24 Alpha power given to the plasma in the generic reactor design 


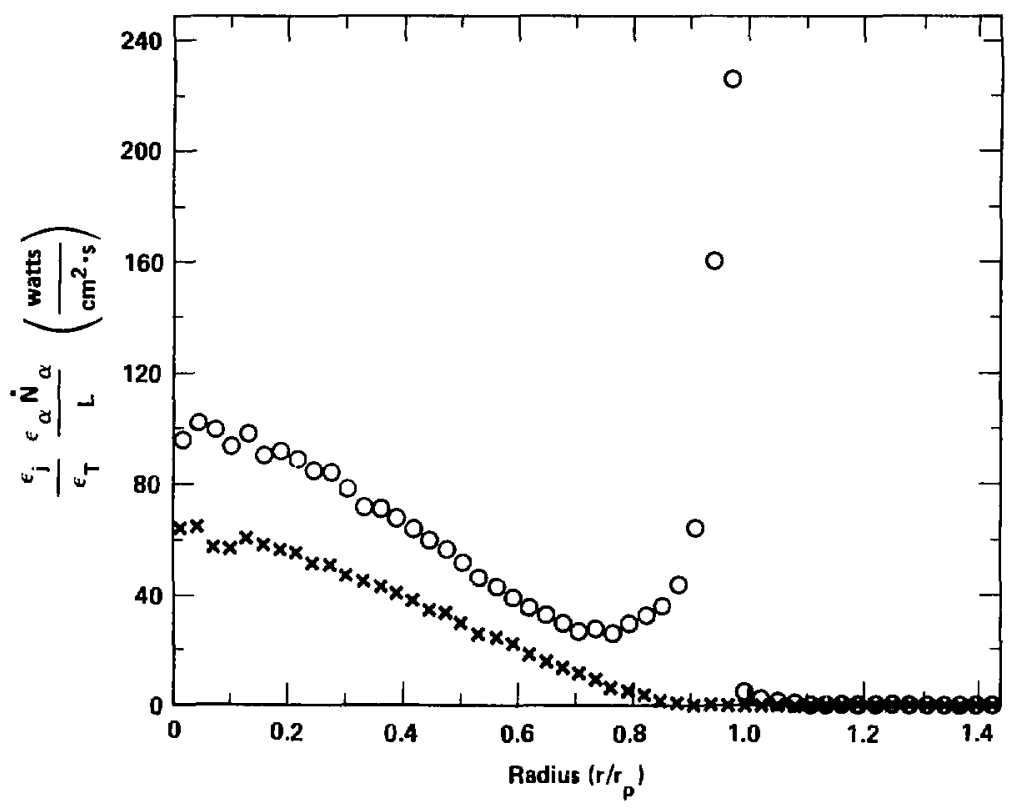

Figure 6.25 Alpha power BIven to plasmi lons ind electrons in the generic reactor design 
corresponds to the edge plasma region where the density and temperature continue to decrease to $T_{1}=T_{e}=50 \mathrm{eV}$ and $n_{1}=n_{e}=5 \times 10^{12} \mathrm{~cm}^{-3}$. The halo region is defined as the non-reactive plasma surrounding the not core, 1.e. $\frac{r}{r_{p}}>0.864$.

The results of generic design study which are presented in Table 6.1 show that $51 \%$ of the alphas thermalize in the reactor core and become "ash" products. These alphas deposit $58 \%$ of their energy in the core, but a slgniflcant amount (32\%) is deposited in the halo. This halo heating will eventually increase the halo temperature, which In turn increases the temperature-profile scale length with respect to $r_{p}$ and $r_{p D}$.

Alpha particle prompt losses have been calculated to be $3 \%$ for the generic deslgn and, therefore, $3 \%$ of the alpha energy is also lost by this mechanism. Beta corrected loss rates are $3 \%$ on axis and $6 \%$ near the plasma edge, and an averagr ' +a of 0.4 gives a loss rate of 5\%. The calculated loss rate of 3\%, sies that the majority of alphas are born in the high beta region of the plasma, and these are the particles that contribute the most to the prompt losses.

The number of alphas that are collislonally lost during slowing down is 10\%. These alphas remove approximately $4 \%$ of the total alpha energy from the plasma, and they leave the plasma with an average energy of $1.4 \mathrm{MeV}$. This Implies that scattering events are becoming Important at alpha energies near $1.4 \mathrm{MeV}$.

Another important quantity is the fraction of alpha energy going to lons and electrons. It has been shown (FIg, 6.2) that the Monte- 
Carlo calculation accurately partitions the alpha energy to each plasma specie according to theory for uniform plasmas. In the present configuration the plasma is far from uniform and more alpha energy goes to the electrons, especially the electrons at the plasma edge. For this reason, the percent of alpha energy that goes to the electrons is 77\%, whereas the lons recelve $21 \%$. These fractions do not add up to $100 \%$ because the final $2 \%$ of the alpha energy is equal to the plasma thermal energy of $60 \mathrm{keV}$.

Finally, we performed a statistical analysis for the nine 1000 particle calculations and compared these values with the one 7000 particle calculation. These results are also glven in Taole 6.1. The calculation for the 7000 particle run takes over 60 minutes of Cray C.P.U. time. Thls run time can be shortened by reducling the number of diagnostics avaliable in the code and by further optimizing the computer program. These technlques can be effective, but the 1000 particle calculations can produce accurate results in approximately 12 C.P.U. minutes. We found that the averages for the 1000 particle runs agree with the 7000 particle run within one standard deviation, and therefore we concluded that 1000 alphas can be used for further reactor studies. Also, the values given in Table 6.1 show that the calculated denslties and energies produced by a 1000 particle MonteCarlo calculation will vary from the mean value by less than $3 \%$, again verifying the accuracy of the 1000 particle runs. 


\subsubsection{Var1ations From the Generio Reactor Des1gn}

The generic reactor design parameters were chosen as representative values which could be used to illustrate the effect of temperature and density gradients on alpha particle confinement. The parameters that directly affect alpha particle confinement are the vacuum magnetic field in the solenoid, beta in the center cell, and the plasma radius. The importance of these three parameters will be 1llugtrated in the following section. The ratio of $p / r_{p}$ is a measure of the plasma's ability to contain the alpha energy, and changes in $B$, $\hat{\beta}$, or $r_{p}$ charige the alpha energy confinement.

\subsubsection{Scale Length Relation for Alpha Energy Conf inement}

Before varying any reactor parameter it is important to determine which of the parameters will affect the alpha energy deposition the most. An approximate way of determining these parameters is by making the following heuristic argument. The alpina deposits its energy along a radial line that extends over two timos its Larmor radius and is given by $\frac{\varepsilon_{\alpha}}{2 p}$. Now the alpha energy which is deposited within the plasma along the plasma diameter is $\frac{N \varepsilon a}{2 r^{*}}$ where $N$ is the total number of alpha particles and $r^{*}$ includes only the alpha energy deposited inside the plasma. In plasmas where the alpha orbit Is smill compared to the plasma diameter, the energy deposited along the diameter can be approximated by $\frac{N \varepsilon_{a}}{2 r_{p}}$. This ratio is proportional to $\frac{\alpha}{2 p}$ so that 


$$
c \frac{\varepsilon}{\alpha \varepsilon_{\alpha}}=\frac{\rho}{r_{p}}
$$

where $\mathcal{C}$ is an arbitrary constant. This ratio was used by Barr, et al. ${ }^{50}$ in studying mirror-confined plasmas that had density temperature profiles that are shown in Fig. 5.5. When $\frac{\left.(2 \mathrm{m \varepsilon})_{\alpha}\right)^{1 / 2}}{\mathrm{qB}_{\mathrm{v}}(1-\langle\beta\rangle)^{1 / 2}}$ is substituted for $\rho$ in Eq. (6.20), then the scale length ratio for energy confinement becomes

$$
\frac{\rho}{r_{p}}=\frac{0.27}{r_{p} B_{v}(1-\langle\beta\rangle)^{1 / 2}}
$$

where $r_{p}$ is in meters and $B_{v}$ in Tesla. The ratio of $\frac{p}{r_{p}}$ defines the important parameters for alpha energy confinement to the plasma radius $\left(r_{p}\right)$, the solenold vacuum magnetic fleld $\left(B_{v}\right)$, and the average plasma beta $\langle\beta\rangle$. Average plasma beta was chosen over $\hat{\beta}$ or $\frac{1}{2} \hat{\beta}$ (as given by Barr, et al.) because 1 t makes $\rho$ proportional to a mean alpha Larmor radlus in the plasma. The fact that the plasma model used in this study is more complex, due to the temperature profile, the inclusion of scattering terms in the collision process, and the addition of the dras gradient drlft, implies that the relation between the parameters in Eq. (6.3.2.1.2) may not be exact. Because of the complexity of finding an exact relation between these parameters, it becomes necessary to pick temperature and density profiles and then change $r_{p}, B_{v}$, or $\hat{B}$ to determine the alpna energy deposition in the plasma. Because $r_{p}$ does 
not necessarily correspond to the hot reacting plasma radius $\left(r_{c}\right)$, we have substituted this value in place of $r_{p}$ in the scale length relation, which becomes $\frac{p}{r_{c}}$.

6.3 .2 .2 Variation of $B, \hat{B}$, ano $r$ for the Single-Scale-Length Analysis The single-scale-length analysis encompasses the results of Monte-Carlo calculations in which the parameters $B_{v}, \hat{B}$, and $r_{p}$ were changed when the temperature and density radial scale lengths are equal, 1.e. $r_{F T}=r_{p D}=r_{p}$. These paraneters were varied such that $0.085 \leq \frac{\rho}{r_{c}} \leq 0.52$ and $n_{e}(0), T_{i}(0)$, and $T_{e}(0)$ were kept equal to the values given for the generic reactor design. The arbitrary $B$ changes are inconsistent with these constant density and temperature values. This Inconsistency enters into the Monte-Carlo simulation through the number of alphas torn at any radial location and in Spitzer's collision model. The radial birth distribution is compensated by calculating alpha density and energy distributions as fractions of the total number of alphas or total alpha energy. As the alpha approaches thermal energy, variations in background plasma properties will affect the collision model, but the relative amount of alpha energy available for the plasma is small, less than $15 \%$ of the $3.52 \mathrm{MeV}$ initlal energy. Therefore, the collision model affects are negligible because small changes in plasma density and temperature do not significaritly affect the drag, angle scatter, and dispersion of a high energy alpha. These arguments imply that the inconsistency of keeping the same values of $n_{e}(0), T_{1}(0)$, and $T_{e}(0)$ for each run introduces second-order effects 
into the caloulation. Also by keeping the on-axis density and temperature fixed for each run, we eliminate the unknown effects in varying too many parameters in our study of alpha thermaljzation. The results of the single-scale-length analysis using the density and temperature profiles presented in Fig. 5.2 are shown in Figs. 6.26 and 6.27. In Fig. 6.26, thermal alpha fractions in the core, halo, and velocity space losses are plotted as a function of $\frac{\rho}{r_{c}}$. Figure 6.27 shows the alpha energy fractions in each of these regions. Each data point in these figures represents a calculation in which 1000 alphas were followed from birth to thermalization. Best-fit curves to the data are also shown. The velocity space losses are modeled using linear regression between $0.085 \leq \frac{\rho}{r_{c}} \leq 0.52$, with the resulting straight lines given by

$$
\begin{aligned}
& q \frac{N_{v}}{N}=-33.6 \frac{\rho}{r_{c}}+24.8 \\
& g \frac{E_{v}}{E}=-20.9 \frac{\rho}{r_{c}}+14.9
\end{aligned}
$$

Halo losses are modeled using a fourth-order polynomial regression between $.085 \leq \frac{\rho}{r_{c}} \leq 0.4$, which are given by

$$
\begin{aligned}
\frac{N_{h}}{N}=-7.97 & +279.78 \frac{\rho}{r_{c}}-3042.61\left(\frac{\rho}{r_{c}}\right)^{2} \\
& +13462.79\left(\frac{\rho}{r_{c}}\right)^{3}=15411.04\left(\frac{\rho}{r_{c}}\right)^{4}
\end{aligned}
$$




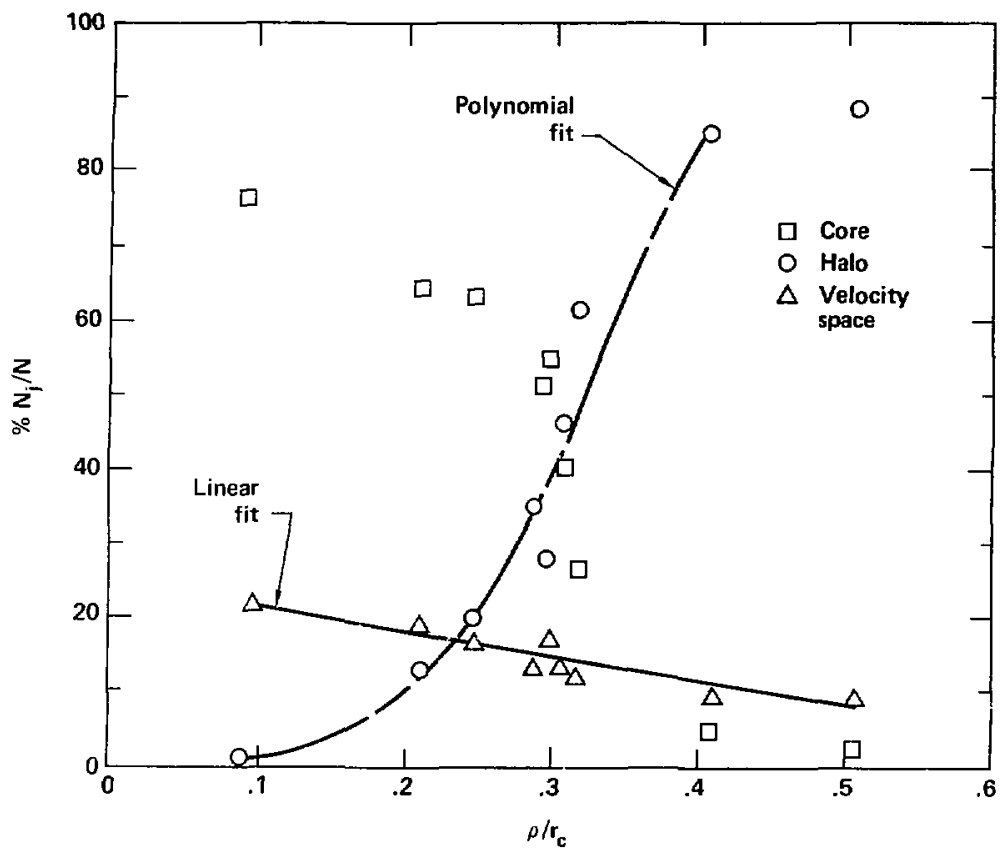

Figure 6.26 Alpha particle distribution to the hot reacting core, halo, and velocity space losses as a function of $\rho / \mathrm{r}_{\mathrm{c}}$ for the single-scale-length problem. The subscript $j$ refers to the core, halo, or velocity space losses. 


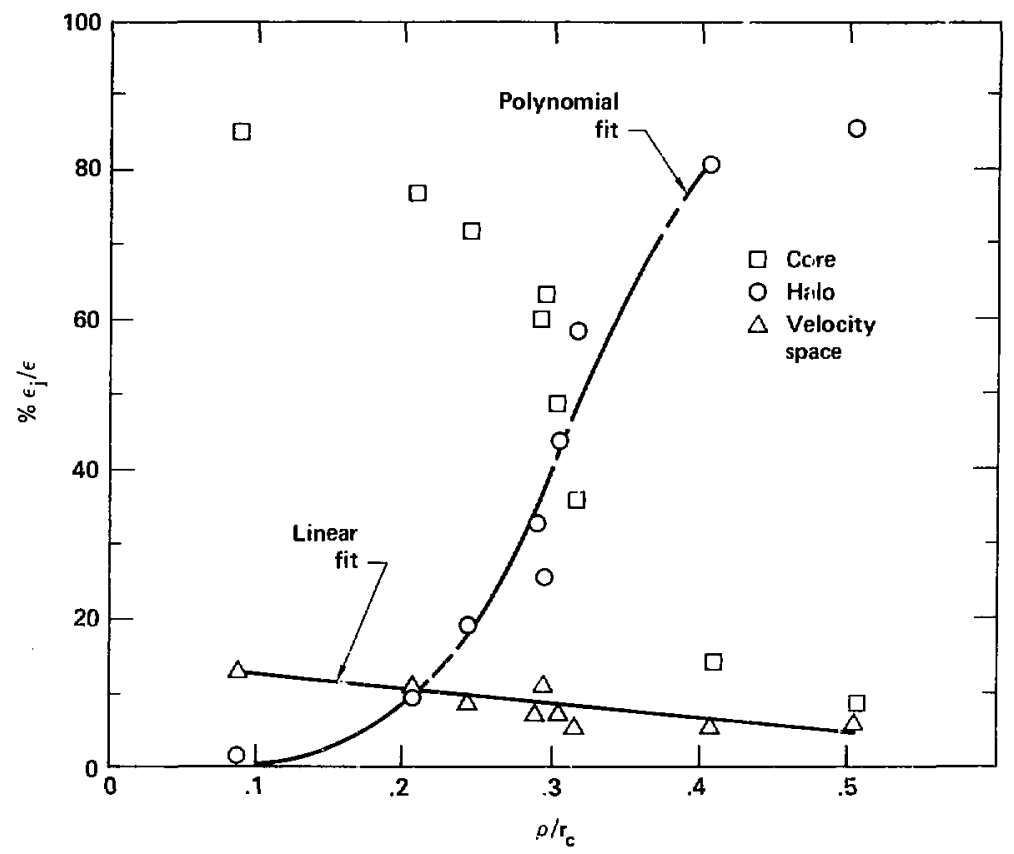

Figure 6.27 Alpha energy distribution to the hot reacting core, halo, and velocity space losses as a function of $\mathrm{o} / \mathrm{r}$ for the single-scale-length problem. The subscrip $j$ refers t,o the core, halo, or velocity space losses. 


$$
\begin{aligned}
q \frac{\varepsilon_{h}}{\varepsilon}=-9.6 \% & +71.87 \frac{\rho}{r_{c}}-806.96\left(\frac{\rho}{r_{c}}\right)^{2} \\
& +7708.13\left(\frac{\rho}{r_{c}}\right)^{3}-10369.29\left(\frac{\rho}{r_{c}}\right)^{4}
\end{aligned}
$$

The fraction that is retained in the core is then

$$
\text { core }=1-\$ \text { halo - } \$ \text { velocity losses }
$$

where the terms core, nalo, and velocity losses refer to elther the alpha or energy fractions.

After obtaining these resulta, several runs were made to determine the effect of changing the density and temperature profile shapes. In the first case, we varied the density profile as shown in Fig. 6.28 and kept the temperature profile constant. The values of $B$, $\hat{B}_{0}$ and $r_{p}$ were chosen so that a comparison would be made with a calculation using a quartic density profile. These comparisons are given in Table 6.2 under the "quartic density" and "modifled denslty" headings. The first difference occurs in $\langle\beta\rangle$, which is $7 \%$ greater for the modified density case, giving a $4 q$ larger value of $\frac{\rho}{r_{c}}$. This density change also caused a $9 \%$ increase in the fractional amount of alphas and alpha energy into the core plasma and a corresponding $9 \%$ deorease in the halo. This is an opposite effert from what we expect. because, as seen from Fig. 6.26 and 6.27 , as $\rho / r_{c}$ increases the fraction of alphas and alpha energy going to the halo increases. 


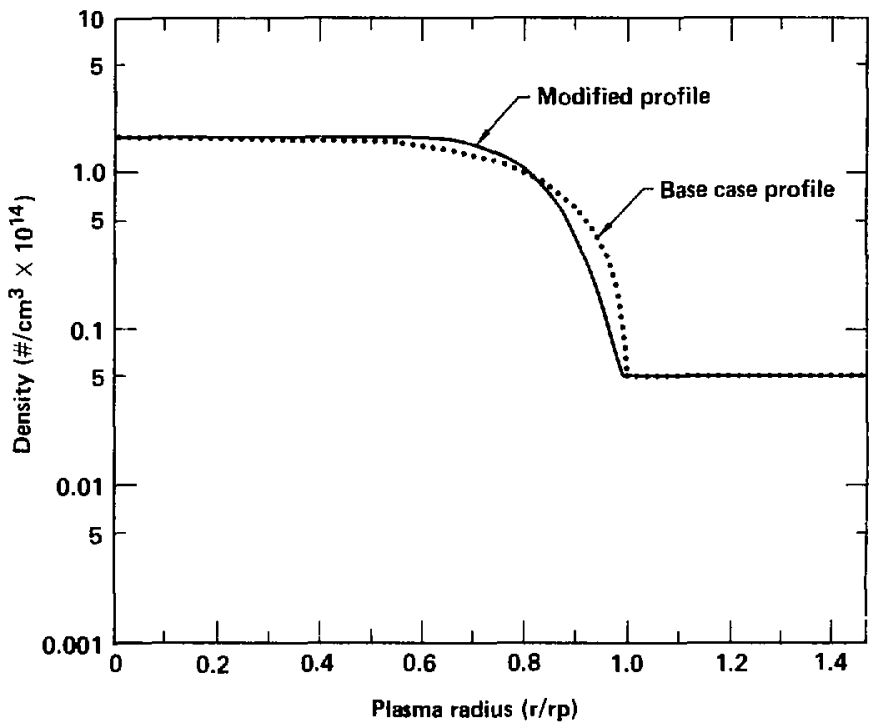

Figure 6.28 Modified density profile for the single-scale-length problem 
Another study was made in which the density and temperature were modeled to correspond to an approximate tenth power (see Eq. 5.2.1) radial proflle. These results are also presented in Table 6.2 , and again $\langle\beta\rangle$ increases so that $\rho / r_{c}$ is 0.327 . As shown in the table, there are more alphas and more alpha energy going into the halo, as

TABLE 6.2

COMPARISON OF MONTE-CARLO SIMULATION RESULTS VARYING

THE DENSITY AND TEMPERATURE PROFILES

Quartic Density and Modified Density and Tenth Power Density Generic Temperature Generic Temperature and Profiles Profiles Temperature Profiles
B (q)
3.0
3.0
3.0
$\hat{B}$ (T)
0.9
0.9
0.9
$r_{p}(m)$
0.5
0.5
0.5
〈 $\beta$ >
0.56
0.6
0.676
$r_{c}(m)$
0.45
0.43
0.48
$\frac{\rho}{r_{c}}$
0.305
0.330
0.327
$\frac{N_{C}}{N}$
40.3
44.0
37.6
$\frac{\mathrm{N}_{\mathrm{H}}}{\mathrm{N}}$
46.2
42.0
51.6
q $\frac{N_{L}}{N}$
13.5
14.0
10.8
$\% \frac{E_{c}}{E}$
48.7
53.0
43.3
$\frac{\varepsilon_{\mathrm{H}}}{\varepsilon}$
43.5
40.0
50.5
$q \frac{E_{L}}{\varepsilon}$
7.8
7.0
6.2 
compared with elther the "quartic density" or "modified density" cases. The increased thermalization in the halo results in a lower thermalization rate in the hot reacting core.

\section{4 Two-Scale-Length Calculation}

The results of the single-scale-length calculation show that when $\rho / r_{c}$ is greater than one-third, there is a significant amount of alpha heating in the halo. Monte-Carlo results show that the alpha energy heats the halo over a narrow radial region near $r_{p}$ and this energy is 1nitially transferred to the halo electrons. The concentration of a large fraction of alpha energy near $r_{p}$ will cause the halo temperature to increase. This in turn will increase the temperature scale length making $\mathrm{r}_{\mathrm{pT}}>\mathrm{r}_{\mathrm{pD}}$, which leads to the twoscale-length problem.

$$
\text { As } \frac{r_{p T}}{r_{p D}} \text { becomes larger than one the radial drift due to drag }
$$

changes. The density effect (see Chapter 5) now occurs before the temperature effect, which can cause an inward radial drift. An inward drift keeps more of the alphas and more alpha energy in the core plasma. The increased temperature scale length also increases the radial region of the hot reacting core plasma to values nearer $\frac{r}{r_{p}}=1$. The effect of increasing $\frac{\mathrm{r}_{\mathrm{pT}}}{\mathrm{r}_{\mathrm{pD}}}$ greater than one changes the alpha energy jeposition in the halo. Table 6.3 compares calculated energy and particle fractions in the hot reacting plasma core and halo for values of $\frac{r_{\mathrm{pT}}}{\mathrm{r}_{\mathrm{pD}}}$ of $1.0,1.1$, and 1.2 . These results were calculated 
TABLE 6.3

COMPARISON OF THE RESULTS FOR THE TWO-SCALE-LENGTH PROBLEM FOR SEVERAL VALUES OF $\mathrm{r}_{\mathrm{pT}} / r_{\mathrm{pD}}$

Quartic Denglty Profiles Modified Density Profile

$\frac{r_{p T}}{r_{p D}}$

${ }_{0}^{N} \frac{C}{N}$ Core

1.0

1.1

1.2

1.2

$\frac{\mathrm{N}_{\mathrm{H}}}{\mathrm{N}}$ Halo

52.0

74.0

78.0

78.0

$\frac{N_{L}}{N}$ Velocity Loss

35.0

9.0

4.0

5.0

E $\frac{E}{E}$ Core

13.0

17.0

18.0

17.0

61.0

81.0

86.0

86.0

$\frac{\varepsilon}{\varepsilon}$ Halo

32.0

9.0

4.0

6.0

$\frac{E_{L}}{\varepsilon}$ Velocity Loss

7.0

10.0

10.0

8.0

$\hat{\beta}$

0.8

0.8

0.8

0.8

B

0.8

0.8

0.8

0.8

$r_{p}$

0.5

0.5

0.5

0.5

〈B

0.53

0.51

0.51

0.61

$\mathrm{r}$

0.43

0.48

0.49

0.48

$\frac{p}{r_{c}}$

0.30

0.27

0.275

0.3

using the generlo design parameters and the density and temperature profiles discussed in Chapter 5 (Fig. 5.2), except that $r_{\mathrm{pT}} \geq r_{\mathrm{pD}}$. Also presented in this table are the results of a calculation in which 
the modifled density profile of F1g. 6.2 and $\frac{r_{p T}}{r_{p D}}=1.2$ are used. The differences in particle and energy distributions between $\frac{r_{p T}}{r_{p D}}=1.1$ and 1.2 are negligible, which Implies that either of these values can be used to study alpha transport for $\frac{r_{\mathrm{pT}}}{r_{\mathrm{pD}}}>1$. A closer look at these two cases (Figs. 6.29 and 6.30 , respectively) shows that when $\frac{r_{p T}}{r_{p D}}=1.1$ the power profile is close to equilibrlum. In F1g, 6.29, the nalo heating peak is low and it is near to the plasma core profile. Therefore, we chose to use the profile ratio of $\frac{r_{p T}}{r_{p D}}=1.1$ for further study. Finally, we note that the modified density profile compares favorably in alpha particle and alpha energy fractions given to the plasma core and halo. This comparison breaks down when we look at 〈B> and $\rho / r_{c}$. We note that the modifled density profile has a $9 \%$ larger value of $\rho / r_{c}$, whlch Implies that there should be a larger number of alpha particles and alpha energy in the halo, with a corresponding decrease in the halo as compared to the quartic density profile case. This result implies that the temperature and density profiles play an important role in the alpha thermalization process.

6.4 .1 Variaticn of $B_{3}, \hat{\beta}_{2}$ and $r_{p}$ for the Two-Saale-Length Problem
We have shown in Section 6.4 that values of $\frac{r_{p T}}{r_{p D}}$ equal to 1.1 produce alpha thermal distributiong that are close to equilibrium. The next step is to vary $B, \hat{B}$, and $r_{p}$, using $n_{e}(0), T_{1}(0)$, and $T_{e}(0)$ equal 


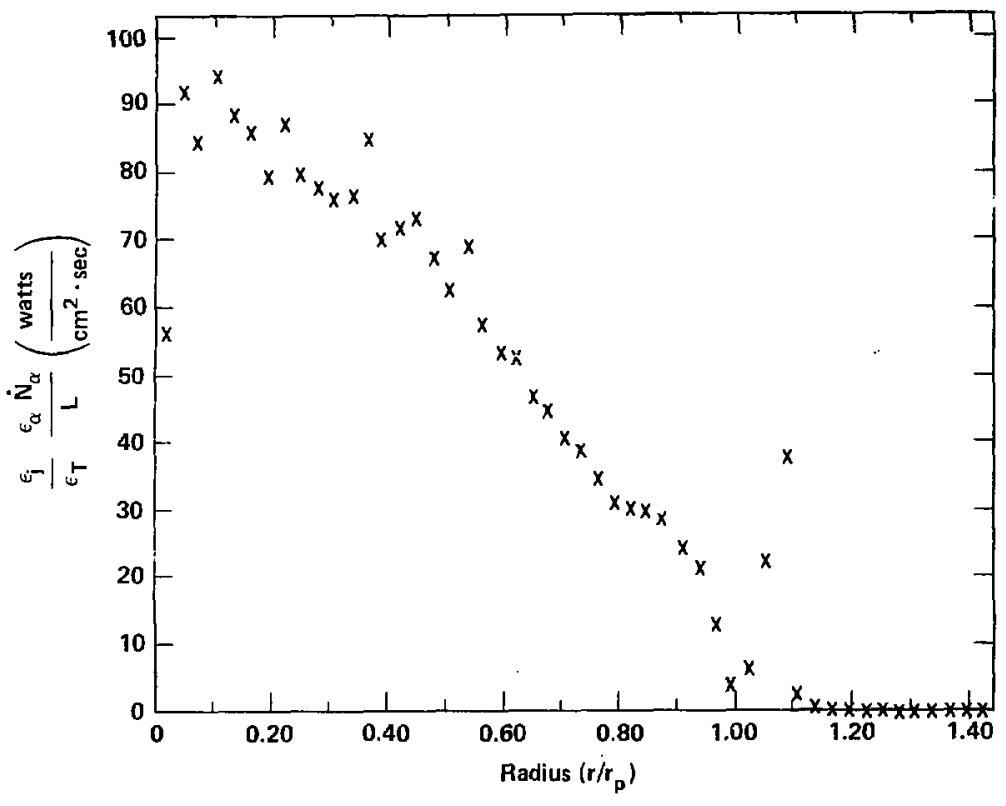

Figure 6.29 Alpha power to the plasma for the two-scale-length problem when $r_{p T} / r_{p D}=1.1$ 


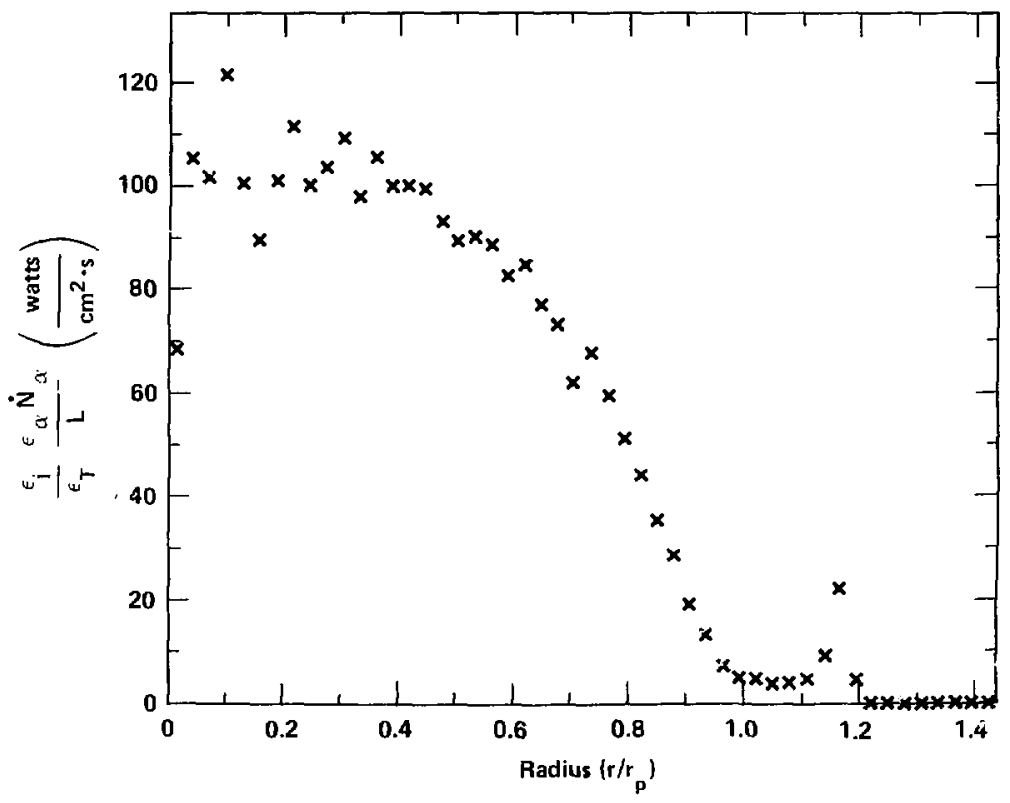

Figure 6.30 Alpha power to the plasma for the two-scale-length problem wher $\mathrm{r}_{\mathrm{pT}} / \mathrm{r}_{\mathrm{pD}}=1.2$ 
to the generic reactor destgn valiues and $\frac{r_{p T}}{r_{p D}}=1.1$. The results of these variations are alpha particle and enerzy fractions to the plasma core and halo that extended over the range $0.085 \leq \frac{p}{r_{c}} \leq 0.59$. This study was composed of 25 Lisdividual Monte-Carlo calculations, each containing 1000 particles. Because of the rumber of Monte-Carlo calculations performed and because of the scatter in the data, we will present the alpha velocity space losses and halo losses as functions of $\frac{\rho}{r_{c}}$ in two separate plots. The fraction of alpha particle and alpha energy deposition into the core can be found from the velocity space and halo losses.

The velocity space lossee include the alphas born in the loss cone and those that pitch angle scattor into the loss cone. These losses are similar to the single-scale-length results and can be inodeled using a linear resression technique. The percent of alpha lost through the loss cone in the range $0.085 \leq \frac{p}{r_{c}} \leq 0.59$ is

$$
\frac{N}{N}=-29.43 \frac{p}{r_{c}}+25.13
$$

and the corresponding energy loss is

$$
\not \frac{\epsilon_{\mathrm{v}}}{\varepsilon}=-16.46 \frac{\rho}{r_{\mathrm{c}}}+14.08
$$


A plot of the energy loss as a function of $\frac{p}{r_{c}}$ is shown in Fig. 6.31. where Eq. (6.4.1.2) 1s shown as the solid line through the Monte-Carlo results. The alpha particle loss is not shown, since 1 t is similar to Fig. 6.31.

The percent of alpha particles thermalizing in the halo and the alpha energy given to the halo for the two-scale analysis follows the same trend as the single-scale-length results. We can therefore model these losses using a fourth-order polymomlal regression technique. The best-fit curve for the alpha particle loss to the halo for $0.085 \leq \frac{p}{r_{c}} \leq$ 0.55 is

$$
\begin{aligned}
q \frac{N_{h}}{N} & =-34.82+743.14 \frac{p}{r_{c}}-5104.90\left(\frac{\rho}{r_{c}}\right)^{2} \quad(6.4 .1 .3) \\
& +13980.97\left(\frac{\rho}{r_{c}}\right)^{3}-11632.16\left(\frac{\rho}{r_{c}}\right)^{4},
\end{aligned}
$$

and the alpha energy loss is

$$
\begin{aligned}
q \frac{\varepsilon_{n}}{\varepsilon} & =-30.49+646.23 \frac{\rho}{r_{c}}-4370.61\left(\frac{p}{r_{c}}\right)^{2} \quad(6.4 .1 .4) \\
& +12027.98\left(\frac{\rho}{r_{c}}\right)^{3}-10039.95\left(\frac{\rho}{r_{c}}\right)^{4} .
\end{aligned}
$$




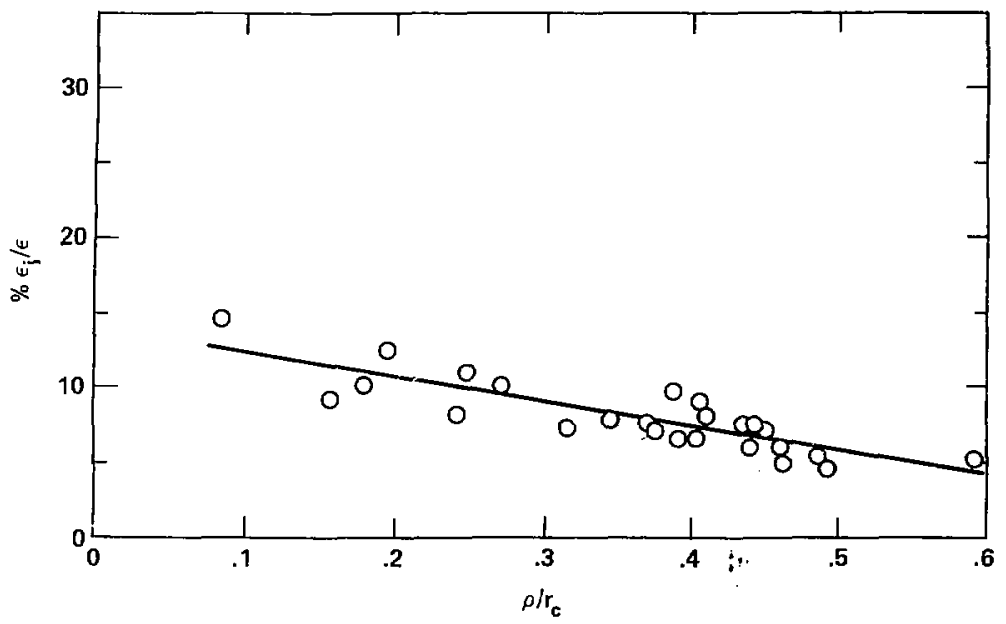

Figure 6.31 Alpha energy losses out of the loss cone for the twoscale-length problem for $r_{\mathrm{pT}} / \mathrm{r}_{\mathrm{pD}}=1.1$ 
The Monte-Carlo calculated energy loss to the halo along with Eq. $(6.4 .1 .4)$ is plotted as a function of $\frac{p}{r_{c}}$ in Fig. 6.32. The alpha particle loss to the halo is similar to Fig. 6.32 and is not shown. The Monte-Carlo data in Fig. 6.32 lyas been plotted so that calculations made with equal values of $B_{v}$ and $r_{p}$ are marked by difierent symbols. We have also connected data polnts in which there were more than two calculations made with the same $B_{v}$ and $r_{p}$. Other data, marked by points, elther vary in $B_{v}, B$, or $r_{p}$ from all the other data shown. We readily observe from this plot that whenever $B_{v}$ or $r_{p}$ ehanges, then equal halo losses do not have the same value of $\frac{p}{r_{c}}$. This implies that the alpha losses to the halo are not simple functions of $\frac{\rho}{r_{c}}$ when $\frac{r_{\mathrm{pT}}}{r_{\mathrm{pD}}}=1,1$, and wo expect th:s result to hold whenever $\frac{r_{\mathrm{pT}}}{r_{\mathrm{pD}}} \neq 1$. Even though there is a lot of scatter in the data in Fig. 6.32, we can approximate the alpha particle and energy contributions to tre plasma core. These can be calculated for the range of $0.085 \leq \frac{\rho}{r_{c}} \leq$ 0.55 by

$$
q \text { core }=100-q \text { halo }-q \text { velocity losses } \quad(6.4 .1 .5)
$$

where the halo amount is calculated from Eq. $(6.4 .1 .3)$ or $(6.4 .7 .4)$ and the corresponding yelocity Iosses from Eq. $(6.4 .1 .1)$ or $(6.4 .1 .2)$.

Finally, we made a comparison between a modeled sixth-power and tenth-power dessity and temperature profile shapes. These comparisons were made using equal values of $B, \hat{B}, r_{p}, n_{e}(0), T_{1}(0)$, and $T_{e}(0)$ and 


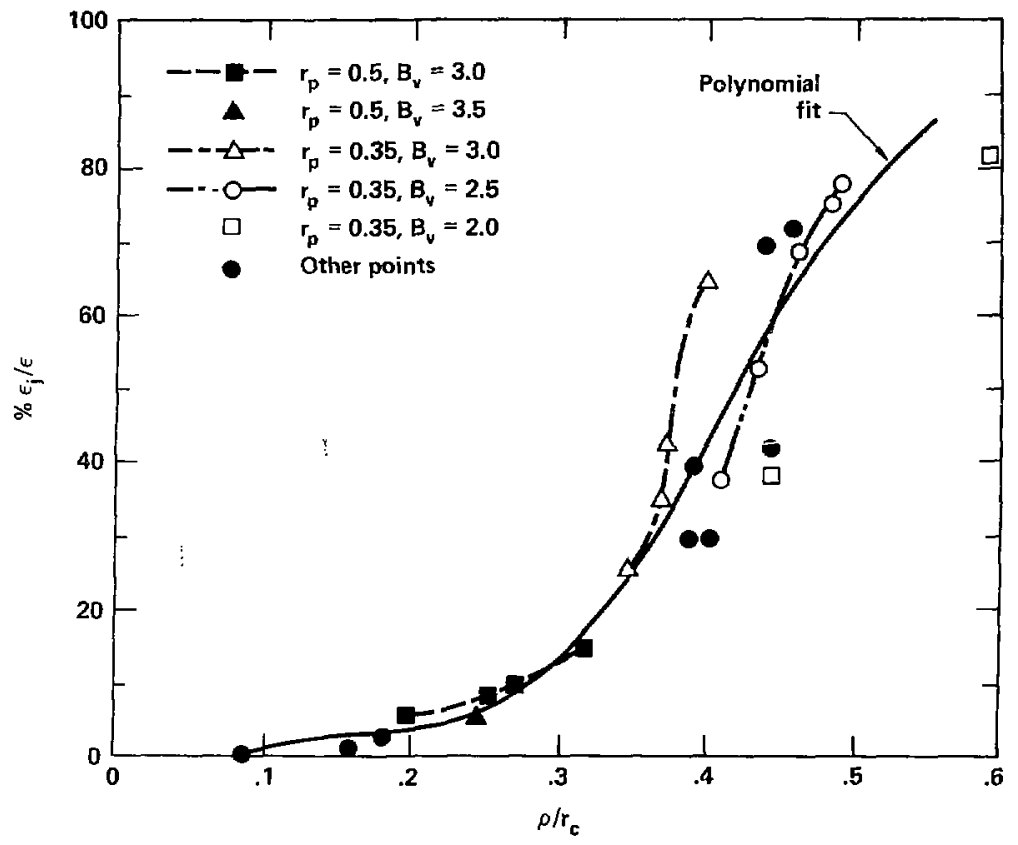

Figure 6.32 Alpha energy losses to the halo for the two-scalelength problem for $\mathrm{r}_{\mathrm{pT}} / \mathrm{r}_{\mathrm{pD}}=1.1$ 
are given in Table 6.4. We can see that as the power of the profile increases, the value of $\langle\beta\rangle$ increases, which increases values of $\frac{\rho}{r_{c}}$. As $\frac{\rho}{r_{c}}$ increased, the fraction of alphas and alpha energy going to the halo increased and the corresponding fractions to the core decreased.

\section{TABLE 6.4}

COMPARISON OF THE RESULTS FOR THE TWO-SCALE-LENGTH PROBLEM USING SIXTH- AND TENTH-POWER DENSITY AND TEMPERATURE PROFILES WITH $\frac{r_{\mathrm{pT}}}{r_{\mathrm{pD}}}=1.1$. Sixth Power Profile Tenth Power Profile

$\begin{array}{lcc}{ }_{\mathrm{pT}} / \mathrm{r}_{\mathrm{pD}} & 1.1 & 1.1 \\ \beta & 0.9 & 0.9 \\ \mathrm{~B}_{\mathrm{r}} & 3.0 & 3.0 \\ r_{\mathrm{p}} & 0.5 & 0.5 \\ \langle B\rangle & 0.623 & 0.717 \\ \mathrm{r}_{\mathrm{c}} & 0.506 & 0.483 \\ \rho / \mathrm{r}_{\mathrm{c}} & 0.29 & 0.35 \\ \% \mathrm{~N}_{\mathrm{c}} / \mathrm{N} \text { Core } & 76.1 & 44.4 \\ \% \mathrm{~N}_{\mathrm{h}} / \mathrm{N} \text { Halo } & 9.7 & 44.2 \\ \% \mathrm{~N}_{\mathrm{L}} / \mathrm{N} \text { Velocity Losses } & 14.0 & 11.4 \\ \% E_{\mathrm{c}} / \mathrm{E} \text { Core } & 84.7 & 51.6 \\ \% E_{\mathrm{h}} / \mathrm{E} \text { Halo } & 8.6 & 41.8 \\ \% \mathrm{E}_{\mathrm{L}} / \mathrm{E} \text { Velocity Loss } & 6.7 & 6.6\end{array}$




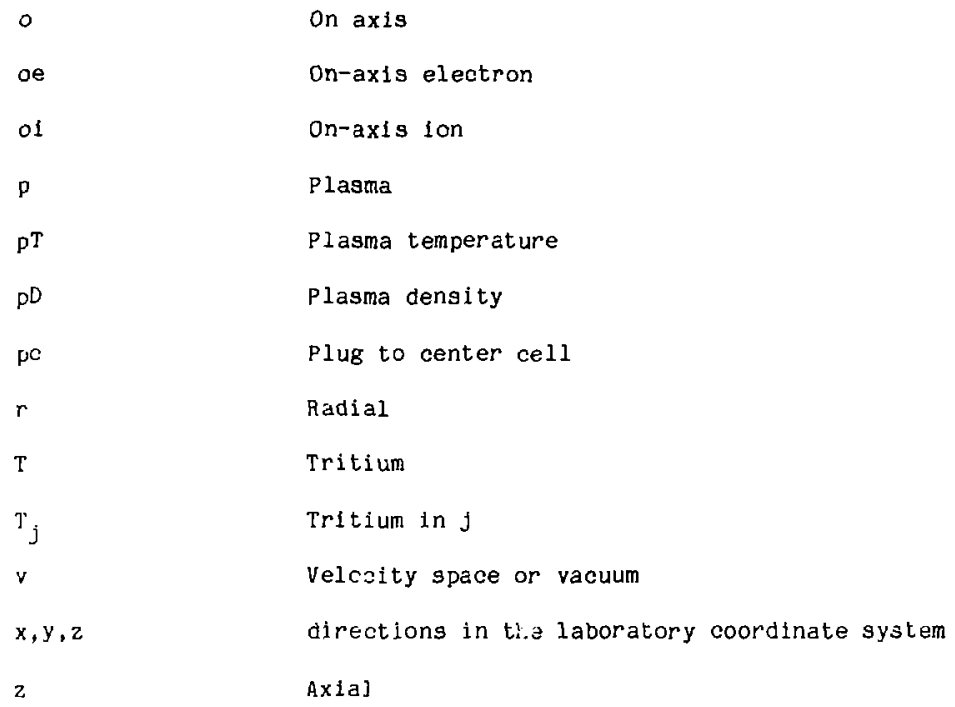

on axis

on-axis electron

On-axis ion

Plasma

Plasma temperature

Plasma density

Plug to center cell

Radial

Tritium

Tritium in $j$

Velcaity space or vacuum

directions in ti.s laboratory coordinate system

Axial

Perpendicular

Parallel

\section{GAEEK SUBSCAIPTS}

Alpha particle

Alpha-alpha

alpha-ion

Energy

Azimuthal angle

Phase angle

P1tch angle

\section{SYMBOLS SUBSCRIPTS}


solution of this problem using the Boltzman equation with the FokkerPlanck collision operator and three plasma species, i.e. electrons, Ions, and alphas, is impossible in the three-dimensional tandem-mirror geometry. Therefore, we have had to rely on density and temperature models that are consistent with present reactor design studies. These profiles have been used to study the effect of varying $B_{v}, \hat{B}$, and $r_{p}$ in a generic reactor design that defines $n_{e}(0), T_{1}(0), T_{e}(0)$, and other pertinent parameters.

We have Investigated several density and temperature profile models, but we have concentrated the majority of the effort in two specific models. The first model, called the single-scale-length problem, uses a quartic density proflle and a higher order temperature profile, both of which have the same scale length. The second model is called the two-scale-length problem because the temperature and density scale lengths differ, but the remaining parameters in these profiles are equal to their counterparts in the singlerscale-length case. In order to compare the results of these studies, we needed to find a common scaling parameter. We have presented a heuristic argument that relates the alpha particle and alpha energy distribution to the core and halo plasma as a function of $\rho / r_{c}$. We have defined $\rho$ to be $\left(\frac{\mathrm{m}}{2} E_{\alpha}\right)^{1 / 2} \frac{1}{\mathrm{qB}_{v}[1-\langle\beta(r)\rangle]^{1 / 2}}$ where $\langle\beta(r)\rangle$ is the average $\beta$ in the plasma core and $E_{\alpha}$ is $3.52 \mathrm{MeV}$. We can find $r_{c}$ from the MonteCarlo output, and it is equal to the radius of the hot reacting core 
plasma. This can be approximated by determining the largest radius, which corresponds to a birth probability of .001 .

After establishing that the alpha halo losses were a function of $f / r_{c}$, we plotted the alpha particle distribution at thermalization and the alpha energy deposition to the core and halo plasma versus $\rho / r_{c}$ for the single-scale-length problem. We also included in this plot the velocity space losses, which Includes the alphas that are immediately lost because they are born in the loss cone plus those that are collisionally lost out the loss cone. The results of this singlescale-length study show that $p / r_{c}$ 1s a good correlation parameter. He also see that when $p / r_{c}>0.3$, the alpha partlcle and energy losses to the halo are greater than 40\%. This, coupled with alpha velocity space losses of $15 \%$ and corresponding energy losses of $9 \%$, shows that there is less than $51 \%$ of the alpha energy avallable for core heating. Iherefore, the single-scale-length results imply that tandem-mirror reactors should be designed with $\rho / r_{c}<0.3$. Fortunately, nature w1ll probably not allow the single-scalelength profiles to exlst in the plasma, especially after the D-T burn has started. We can say this because the results of the Monte-Carlo code, which produces equilibrium profiles, shows a very sharp peak in both alpha density and alpha energy that occurs in a narrow radial region of the halo for the single-scale-length problem. These sharp peaks will relax to some equilibrlum condition which does not affect the D-T density proflle, but it will increase the radial scale length of the temperature profile. We have investigated this possibility by 
first choosing the ratio of temperature to density scale lengths $\left(\frac{\mathrm{pT}}{\mathrm{r}_{\mathrm{pD}}}\right)$, such that alpha density and energy proflles are close to equilibrium. This ratio of scale lengths used in this study was $\frac{r_{\mathrm{pT}}}{r_{\mathrm{pD}}}=1.1$ Next, we changed values of $B, \hat{B}$, and $r_{p}$ while keeping the other generic reactor parameters fixed and using $\frac{r_{\mathrm{pT}}}{r_{\mathrm{pD}}}=1.1$ to study the alpha thermalization process. These results show that the functional relation between the alpha losses and $p / r_{c}$ is not as simple as the single-scale-length case. Data scatter of $50 \%$ can be observed for some values of $p / r_{c}>0.35$. We assume that this scatter is related to the alpha guiding center birth distribution because this gulding center distribution will also fluctuate depending on the size of the Larmor orbit compared to the radial scale length. Unfortunately, we have not been able to solve this problem at this time and we must leave it for future work.

Although we noted a signiflcant amount of data scatter for the two-scale-length problem, we have nevertheless obtained a fourth-order best-fit curve to this data. From this curve we can generalize that when $\rho / r_{c} \geq 0.39$, then $40 \%$ or more of the alphas and alpha energy is lost to the halo. This is only a slight improvement in the parameter $\rho / r_{c}$ as compared to the single-scale-length results. We must also point out that that large data scatter $1 \mathrm{mplies}$ that exact results depend on the chosen values of $B, \hat{B}$, and $r_{p}$. 
We have also done less extensive studies varying the density and temperature profiles for $g$ iven values of $B, \hat{\beta}$, and $r_{p}$. We have done this for both the single-scale-length and two-scale-length problems. The results indlcate that specific reactor designs are affected by the density and temperature profiles that are used. These profiles affect the alpha birth profile, $\beta$ profile, the drag drift, and therefore the alpha thermal particle and alpha energy deposition profiles. We note that changes in the density and temperature profiles follow tha same trends as discussed for the single- and two-scale-length problems, but more reliable results can only be obtained from specific calculations, especially when $\mathrm{p} / \mathrm{r}_{\mathrm{c}}>0.3$.

We can summarize the results of this study with the following statements. The most general result is that valies of $\rho / r_{c}$ greater than 0.3 produce excessive alpha energy loss to the halo. Secondly, the particular alpha particle and energy distritiutions after thermalization can only be determined by knowing the plasma density and temperature proflles along with the specific plasma parameters. Specific plasma profiles and parameters must be used as input to an alpha thermalization code, such as the one used in this study, to determine the alpha distributions. If the resulting alpha distributions are not in equiliorium, then changes have to be made to the original density and temperature profiles and then the calculation is performed again. This iterative process is necessary because of the complexity oi solving the Boltzman equation self-consistently for this problem. 
It is approprlate at this point to briefly review the areas where this study could be either improved or used. Theoretical work needs to be jone to determine the limiting radial magnetic field gradients that can be used to meet adiabaticity conditions and therefore instill more confidence in the use of the guiding center approximation when radial gradients are present. Next, the radial drag drift rate is a complex calculation that requires an excessive amount of computer time; this scheme should be improved, if possible. Work is also needed to improve the alpha particle and energy correlation to the correct functional form of $\rho / r_{c}$ so the data scatter in the two-scalelength problem is reduced. Finally, it is well known that any computer code generated to solve a complex problem cannot be guaranteed to be completeiy accurate in every application. Therefore, there are coding improvements and then further tests that could be made to guarantee the reliability and accuraoy of this Monte-Carlo calculation.

As far as applying this Monte-Carlo code to other applications, we could modify this code to address the alpha thermalization process in tokamak fusion plasmas that are surrourjed by an edge (halo) plasma. In a more related area, we could study alpha trapping rates into the thermal barpler and the assoclated alpha pumping problem. We could also address alpha transport in $D-T$ driven mirror machines, which has applications toward advancing fusion technology. Other applications, such as studying fast ions, or other products in a tandem-mirror reactor, are also possible. 
In conclusion, we have generated a tool that has been used to study alpha particle transport during the thermalization process in tandem-mirror reactors. This tool is a Monte-Carlo Code which incorporates the important physical processes that affect alpha thermalization. 


\section{PAat 8}

\section{LITERATURE CITED}

1. C.C. Damm, "Evolution of the Mirror Machine," Lawrence Livermore National Laboratory, Livermore, CA, UCRL-89277, 1983.

2. A.S. Bistop, "Project Sherwood," Addison Wesley, Reading, Mass., 1958.

3. M.M. El-Wakil, Nuclear Power Sngineering, McGraw-Hill, 1962.

4. F.F. Chen, "Introduction to Plasma Physics," Plenum Press, 1976.

5. T.K. Fowler, B.G. Logan, Comments Plasma Phys. Cont. Fus. 3 (6), $167,1977$.

6. G.I. Dimov, V.v. Zalaidakov, M.E. Klshinerskii, Fiz. Plazmy 2, 597, 1976; [Sov. I Plasma Phys. 2 (4), 326, (1976)].

7. D.J. Rose, Nuclear Fuston 9, 183, 1969.

8. M. Ohnishi, H. Tokunaga, F. Hakabayashi, Nuclear Fusion 16 (4), $690,1976$.

9. Ya.I. Kolesnichenko, Nuclear Fusion $20(6), 727,1980$.

10. D.G. McAlees, "Alpha Particle Energetics and Neutral Beam Heating in Tokamak Plasmas," Oak Ridge National Laboracory, Oak Ridge, TN, ORNL-TM-4661, 1974.

11. M. Ohntshi, N. Ao, J. Wakabayashi, Nuclear Fusion 18 (6), 859, 1978.

12. H. Tsuji, M. Kasurai, T. Sekiguchi, Nuclear Fusion 16 (2), 287 , 1976.

13. D.E. Post, "Some Aspects of Alpha Particle Heating," Princeton Plasma Physics Laboratory, Princeton, NJ, PPPL-1815-UC-20G, 1981 .

14. K. Tani, T. Takizuka, M. Azumi, H. Kishimoto, Nuclear Fusion 23 (5), 657, 1983.

15. L.M. Hively, G.H. Miley, Nuclear Fusion 17 (5), 1031, 1977.

16. D.E. Drtemeyer, "A Study of Fusion Product Effects in Field Reversed Mirrors," University of Illinois, Fusion Studies Laboratory, Urbana, IL, COO-2218-150/EPRI-AFR-20, 1980, . 
17. R.E. Potok, L.M. Lidsky, P.A. Politzer, "Particle Confinement and Diffusion in Torsations," Massachusetts Institute of Technology, Plasma Fusion Center, Boston, MA, PFC/RR-80-15, 1980.

18. R.W. Moir, W.L. Barr, G.A. Carlson, W.L. Dexter, J.N. Doggett J.H. Eink, G.W. Hamilton, J.D. Lee, B.G. Logan, W.S. Neef, Jr., M.A. Peterson, and M.E. Rensink, "Preliminary Design Study of the Tandem Mirror Reactor (TMR), "Lawrerce Livermore National Laboratory, Livermore, CA, UCRL-52302, 1977.

19. R.S. Devoto, M. Ohnishi, "Confinement of Alpha Particies in Tandem Mirrors," Lawrence Livermore National Laboratory, Livermore, CA, UCID-18784, 1980.

20. R.S. Devoto, M. Ohnishi, J, Kerns, J.T', Woo, "Alpha Particle Conf inemert in Tandem Mirrors," Lawrence Livermore National Laboratory, Livermore, CA : UCRL-84289, 1980.

21. J.F. Santarius, and J.D. Callen, "Alpha Particle and Energy Deposition in Tandem Mirrors," University of Wisconsin, Madison, WI, UFWDM-425, 1981.

22. J.F. Santarius, "Halo Plasma Physics Model for Mirror Machines with Neutral Beam Injection," University of 'Iisconsin, Madison, WI, UWFDM-598, 1985.

23. W.L. Hsu, W. Eauer, R.A. Kerst, K.L. Wilson, "Halo Plasma Heating by Neutral Beam Injection in TMX-U," Sandia National Laboratory, Livermore, CA, SAND84-8906, 1984.

24. B.J. Braams, and C.E. Singer, "Low Temperature Plasma Near a Tokamak Reactor Limiter," Princeton Plasma Physics Laboratory, Princeton, NJ, PPPL-2191, 1985.

25. Y.B. Gott, M.C. Ioffe, T.G. Telkovsky, Nuclear Eusion Supplement, Part 3, 1045, 1962.

26́. V.P. Pastukhov, Nuclear Fusion 14, 3, 1974.

27. E.H. Coensgen, W.F. Cumrins, B.G. Logan, A.W. Molvik, W.E. Nexsen, T.C. Simonen, B.W. Stallard, W.C. Turner, Proc. 7 th Eur. Conf. Cont. Fusion Plasma Phys., 1975, vol, II, 167.

28. D.D. Ryutov, G.V. Stupakov, Sov. Phys. Dokl. 23 (6), $412,1978$.

29. D.E. Baldwin, Logan, B.G., Phys. Rev. Lett. 43 (5), 1318, 1979.

30. N.A. Krall, and A.W. Trivelpiece, "Principle of Plasma Physics," McGraw-Hill, 1973. 
31. M.N. Rosenbluth, H.M. MacDonald, D. Judd, Phys, Rev., 107 (1), 1, 1957.

32. L. Spltzer, "Physics of Fully Ionized Gases," Interscience Pub. 1962.

33. J. Kesner, G. Shuy, Y.Z. L1, C.W. Lee, "Super-Tara, A Wall Stabilized Reactor Study," Plasma Fusion Center, Massachusetts Institute of Technology, Bostcn, MA.

34. R.S. Devoto, D.E. Baldwin, B.G. Logan, G.W. Harklton, B.W. Johnson, "A Small, Octopole - Stabilized Tandem Misror Reactor," Lawrence Livermore National Laboratory, Livermore, CA, UCID-20157, 1984.

35. E.B. Hooper, D.E. Baldwin, I.K. Fowler, R.J. Kane, H.C. Turner, Phys. Fluids $\underline{27}(9), 2264,1984$.

36. S.J. Sackett, "EFFI - A Code for Calculating Electromagnetic Field, Force, and Inductance in Coll Systems of Arbltrary Geometry," Lawrence Livermore National Laboratory, Livermore, CA, UCID-17621, 1977.

37. J.C. Riordin, A.J. Lichtenberg, M.A. Lieberman, Nuclear Fusion 19 (1), 21, 1979 .

38. T.B. Kalser, "MHD Stability," Physles Basis for MFiF-B, Sec. IVB, Lawrence Livermore National Laboratory, Liveriuore, CA, UCID-18496, Part 2, 1980.

39. R.H. Cohen, G. Rowlands, and J.H. Foote, Phys. Fluide 21 (4), 627.1978.

40. T.G. Northrop, "The Adlabatic Motion of Charged Particles," Interscience Publishers, 1973.

41. T.H. Johnson, "Gulding Center Simulations of Toroidal Plasmas," Lawrence Livermore National Laboratory, Livermore, CA, UCRL $-51725,1974$.

42. C. Tull, "Gulding Center Simulations of Strong Ion Beams with Applications to the Counterstreaming Ion Torus, "Lawrence L1 vermore National Laboratory, L1 vermore, CA, UCHL -52436 , 1978.

43. D.V. Sivukhin, "Coulomb Collisions in a Fully-Ionized Plasma," Reviews of Physics, Consultants Bureau, New York, $4,93-241$, 1966. 